Beiträge aus der Nordwestdeutschen Forstlichen Versuchsanstalt Band 1

\title{
Clusterstudie Forst und Holz Niedersachsen
}

Burkhard Rüther, Jan Hansen, Agatha Ludwig, Hermann Spellmann, Jürgen Nagel, Bernhard Möhring, Matthias Dieter
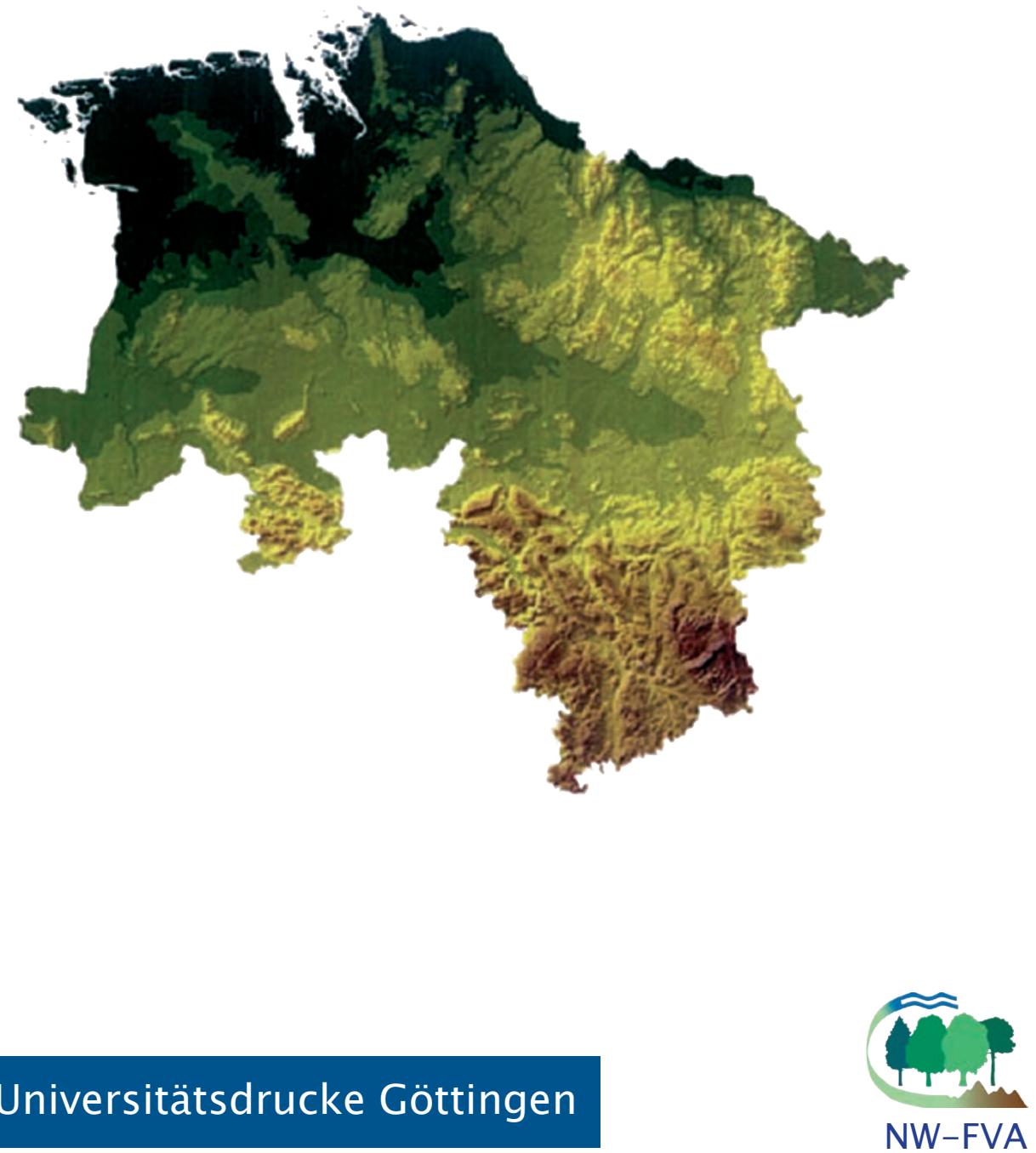

Burkhard Rüther, Jan Hansen, Agatha Ludwig, Hermann Spellmann, Jürgen Nagel, Bernhard Möhring, Matthias Dieter

Clusterstudie Forst und Holz Niedersachsen

Except where otherwise noted, this work is licensed under a Creative Commons License 
erschienen als Band 1 der Reihe „Beiträge aus der Nordwestdeutschen Forstlichen Versuchsanstalt“ in den Universitätsdrucken im Universitätsverlag Göttingen 2007 
Burkhard Rüther, Jan Hansen, Agatha Ludwig, Hermann Spellmann, Jürgen Nagel, Bernhard Möhring, Matthias Dieter

\section{Clusterstudie \\ Forst und Holz \\ Niedersachsen}

Beiträge aus der

Nordwestdeutschen

Forstlichen Versuchsanstalt

Band 1

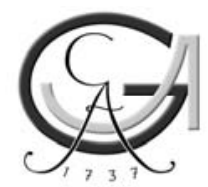

Universitätsverlag Göttingen 2007 


\title{
Bibliographische Information der Deutschen Nationalbibliothek
}

Die Deutsche Nationalbibliothek verzeichnet diese Publikation in der Deutschen Nationalbibliographie; detaillierte bibliographische Daten sind im Internet über $<$ http://dnb.ddb.de> abrufbar.

Global Forest Decimal Classification: GFDC 721.1, 906, (430)

\author{
Herausgeber \\ Nordwestdeutsche Forstliche Versuchsanstalt (NW-FVA) \\ Grätzelstr. 2, D-37079 Göttingen \\ Tel.: +49 (0)551-69401-0, Fax: +49 (0)551-69401-160 \\ E-Mail: zentrale@nw-fva.de \\ www.nw-fva.de \\ Schriftleitung der Reihe: Prof. Dr. Hermann Spellmann \\ Redaktion: Inge Kehr, Ulrike Gaertner
}

Die Clusterstudie Forst und Holz Niedersachsen wurde vom Niedersächsischen Ministerium für den ländlichen Raum, Ernährung, Landwirtschaft und Verbraucherschutz finanziert und als Gemeinschaftsprojekt der Nordwestdeutschen Forstlichen Versuchsanstalt - Abteilung Waldwachstum -, des Burckhardt-Institutes der Universität Göttingen - Abteilung Forstökonomie und Forsteinrichtung - und des Niedersächsischen Kompetenznetzes für Nachhaltige Holznutzung (NHN) durchgeführt.

Dieses Buch ist auch als freie Onlineversion über die Homepage der NW-FVA, des Verlages sowie über den OPAC der Niedersächsischen Staats- und Universitätsbibliothek (http://www.sub.uni-goettingen.de) erreichbar und darf gelesen, heruntergeladen sowie als Privatkopie ausgedruckt werden. Es gelten die Lizenzbestimmungen der Onlineversion. Es ist nicht gestattet, Kopien oder gedruckte Fassungen der freien Onlineversion zu veräußern.

Titelabbildung: Landesvermessung und Geobasisinformation Niedersachsen (LGN)

C 2007 Universitätsverlag Göttingen

http://univerlag.uni-goettingen.de

ISBN-13: 978-3-940344-07-6

ISSN: $\quad$ 1865-6994 


\section{Vorwort}

\section{Cluster Forst und Holz in Niedersachsen \\ - Bedeutung, Aussichten und Chancen -}

In Niedersachsen sind 1,1 Millionen Hektar bzw. $24 \%$ der Landesfläche mit Wald bedeckt. Damit ist der Wald ein prägendes Element der niedersächsischen Landschaft. Er erfüllt wichtige Schutz- und Erholungsfunktionen und liefert den vielseitig verwendbaren Rohstoff Holz, bei dessen Produktion das klimaschädliche Treibhausgas $\mathrm{CO}_{2}$ gebunden und Sauerstoff freigesetzt wird.

Gerade in einer Zeit, in der sich die Gesellschaft der zunehmenden Verknappung von Ressourcen und der schädlichen Auswirkungen der Verbrennung fossiler Brennstoffe auf die Umwelt und das Klima bewusst wird, spielt der Wald und das in ihm wachsende Holz eine immer größer werdende Rolle.

Die Einführung des Gedankens der Nachhaltigkeit in die Forstwirtschaft und die Aufbauleistungen der letzten Jahrzehnte haben dazu geführt, dass sich der Vorrat heute auf einem bisher nicht erreichten Niveau befindet und vermehrt Holz aus heimischen Wäldern genutzt werden kann. Die Holzverarbeitung schafft Arbeitsplätze, besonders in mittelständisch strukturierten Handwerks- und Zuliefererbetrieben, und stärkt damit den ländlichen Raum.

Der Weg des Rohstoffes Holz aus den Forstbetrieben direkt oder über den Holzhandel in die holzbearbeitenden bzw. holzverarbeitenden Betriebe bis hin zum Endverbraucher stellt eine bedeutende Wertschöpfungskette dar. Die an dieser Wertschöpfungskette beteiligten Unternehmen in Niedersachsen bilden den Cluster Forst und Holz. Er ist ein bedeutender Wirtschaftsfaktor im eigenen Bundesland und über die Landesgrenzen hinaus.

Die vorliegende Clusterstudie zeigt die Verflechtungen innerhalb der Forstund Holzbranche auf, prognostiziert das künftige Holzaufkommen und schafft die Grundlagen für eine intensive Diskussion zwischen Politik, Wirtschaft und Wissenschaft über Bedeutung, Aussichten und Chancen der Gesamtbranche.

Beiträge aus der NW-FVA, Band 1, 2007 
Moderne Clusterpolitik lebt von der aktiven Beteiligung aller Marktpartner an einer progressiven Weiterentwicklung der gemeinsamen Wertschöpfungskette. Ich wünsche mir, dass die vorliegende Clusterstudie Forst und Holz in Niedersachsen ein positiver Einstieg in die strategische Ausrichtung der Forst- und Holzbranche sein wird.

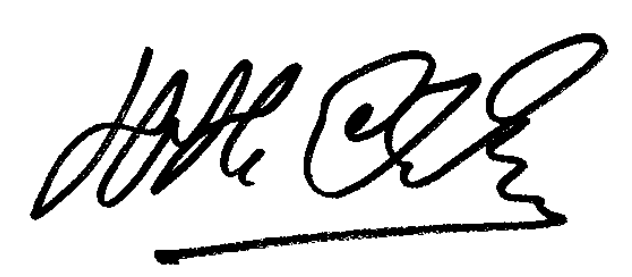

Hans-Heinrich Ehlen

Niedersächsischer Minister für den ländlichen Raum, Ernährung, Landwirtschaft und Verbraucherschutz 


\section{Inhaltsverzeichnis}

Vorwort I

Inhaltsverzeichnis III

Clusterstudie Forst und Holz Niedersachsen 1

1 Einleitung $\quad 5$

1.1 Anlass und Ziele 5

$\begin{array}{ll}1.2 \text { Hintergrundinformationen } & 7\end{array}$

1.2.1 Stellung der niedersächsischen Forstwirtschaft im nationalen Vergleich 7

1.2.2 Globalisierung des deutschen Holzmarktes 9

2 Der niedersächsische Cluster Forst und Holz 13

$\begin{array}{ll}2.1 \text { Allgemeines } & 13\end{array}$

2.2 Material und Methoden 14

2.2.1 Erhebung von Umsatz und Unternehmensanzahl 14

$\begin{array}{ll}\text { 2.2.2 Erhebung der Beschäftigten } & 16\end{array}$

$\begin{array}{ll}\text { 2.2.3 Volkswirtschaftliche Gesamtrechnung } & 16\end{array}$

$\begin{array}{ll}2.2 .4 & \text { Sonstige Quellen } \\ & 17\end{array}$

$\begin{array}{lll}2.3 \text { Ergebnisse } & 17\end{array}$

$\begin{array}{ll}\text { 2.3.1 Entwicklung der Jahresumsätze } & 17\end{array}$

$\begin{array}{ll}\text { 2.3.2 Entwicklung der Unternehmenszahlen } & 21\end{array}$

$\begin{array}{ll}\text { 2.3.3 Entwicklung der Beschäftigtenzahlen } & 23\end{array}$

$\begin{array}{ll}\text { 2.3.4 Die volkswirtschaftliche Gesamtrechnung } & 27\end{array}$

2.4 Darstellung der (über-)regionalen Rohholzströme Niedersachsens 29

$\begin{array}{ll}2.4 .1 \text { Allgemeines } & 29\end{array}$

$\begin{array}{ll}2.4 .2 \text { Sägefähiges Nadelholz } & 30\end{array}$

$\begin{array}{ll}2.4 .3 & \text { Nicht sägefähiges Nadelholz } \\ 2.4 .4 & 32\end{array}$

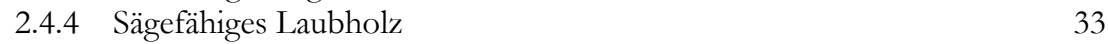

$\begin{array}{ll}\text { 2.4.5 Nicht sägefähiges Laubholz } & 34\end{array}$

2.5 Bedeutung der energetischen Holznutzung in Niedersachsen 35

3 Analyse des Nutzungsverhaltens zwischen BWI 1 und BWI $2 \quad 37$

3.1 Allgemeines 37

3.2 Datengrundlage 37

3.3 Vergleich der durch BWI 1 und BWI 2 dokumentierten Waldzustände und $\begin{array}{ll}\text { Nutzungsintensitäten } & 38\end{array}$

3.3.1 Vorrats-, Alters- und Grundflächenveränderung zwischen 1987 und 2002

3.3.2 Vergleich der Altersklassenstruktur des Vorrates in Beständen mit vorherrschender Buche

3.3.3 Vergleich der Altersklassen- und Durchmesserklassenstruktur des Vorrates in Beständen mit vorherrschender Eiche 
3.3.4 Vergleich der Altersklassen- und Durchmesserklassenstruktur des Vorrates in Beständen mit vorherrschendem Laubholz niedrigen Umtriebs

3.3.5 Vergleich der Altersklassen- und Durchmesserklassenstruktur des Vorrates in Beständen mit vorherrschender Fichte

3.3.6 Vergleich der Altersklassen- und Durchmesserklassenstruktur des Vorrates in Beständen mit vorherrschender Kiefer

3.4 Einflussfaktoren auf das Nutzungsverhalten zwischen 1987 und 2002

3.5 Statistische Analyse des Nutzungsverhaltens $\quad 50$

4 Forstliche Nutzungspotenziale $\quad 59$

$\begin{array}{ll}\text { 4.1 Material und Methoden } & 59\end{array}$

4.1.1 Vorstellung der Prognosewerkzeuge $\quad 60$

4.1.2 Startwerte $2007 \quad 60$

4.1.3 Waldentwicklungsszenarien $\quad 63$

4.1.4 Sortierungsvorgaben $\quad 64$

4.2 Forstliche Nutzungspotenziale in Niedersachsen bis 2036 bei unterschiedlichem Nutzungsverhalten $\quad 65$

4.2.1 Entwicklung der Holzvorräte $\quad 65$

4.2.2 Entwicklung der Zuwächse und Nutzungen $\quad 67$

4.2.3 Baumartenspezifische Betrachtung der Nutzungen 69

$\begin{array}{lll}\text { 4.2.4 Sortenertrag } & 73\end{array}$

$\begin{array}{ll}\text { 4.2.5 } & \text { Berücksichtigung eigentümerspezifischer und naturaler } \\ \text { Rahmenbedingungen } & 75\end{array}$

5 Schlussfolgerungen $\quad 79$

6 Ausblick $\quad 85$

$\begin{array}{llr}7 & \text { Literatur } & 87\end{array}$

Glossar $\quad 91$

$\begin{array}{ll}\text { Autoren } & 92\end{array}$ 


\section{Clusterstudie Forst und Holz Niedersachsen}

\section{Forest and Wood Cluster Study, Lower Saxony}

\section{Zusammenfassung}

Die Globalisierung der Rohstoff- und Warenmärkte stellt die deutsche Forst- und Holzwirtschaft vor neue Herausforderungen. Sie hat zu einer steigenden Holznachfrage und zum Aufbau neuer Produktionskapazitäten der Holzindustrie geführt. Ziel der Clusterstudie Forst und Holz Niedersachsen ist es, die Wettbewerbsfähigkeit der niedersächsischen Forst- und Holzwirtschaft vor diesem Hintergrund zu stärken. Hierzu wurden die inneren Strukturen (Betriebe, Umsätze, Beschäftigungszahlen) und wirtschaftlichen Potenziale analysiert, die möglichen Holzaufkommen bei Unterstellung verschiedener Bewirtschaftungsstrategien für einen Zeitraum von 30 Jahren prognostiziert und die wesentlichen Einflussfaktoren auf das zu erwartende Nutzungsverhalten der Forstbetriebe aufgezeigt.

Niedersachsen hat bundesweit die drittgrößte Waldfläche. Der hohe Kleinprivatwaldanteil und ungünstige Altersstrukturen erschweren eine stetige Holznutzung. Mit einem Umsatz von ca. 15 Mrd. $€$ im Jahre 2005 betrug der Beitrag des niedersächsischen Forst und Holz Clusters zum steuerlich ausgewiesenen Gesamtumsatz des Landes 3,4\%. Mit diesem Umsatz steht der niedersächsische Cluster im bundesweiten Vergleich immer noch an 4. Stelle, obwohl in zahlreichen anderen Bundesländern neue Produktionskapazitäten der Säge- und Holzwerkstoffindustrie aufgebaut wurden.

Die Ergebnisse der Holzaufkommensprognosen auf der Basis der drei Waldentwicklungsszenarien „naturnaher“, „ertrags-“ und „naturschutzorientierter“ Waldbau weisen darauf hin, dass in bereits heute intensiv nutzenden Forst- 
betrieben mit einem guten Pflegezustand sich durch eine Modifikation der Waldbaustrategien kaum noch zusätzliche Rohholzmengen mobilisieren lassen, ohne die Nachhaltigkeit zu gefährden. Den wichtigsten Ansatzpunkt zur Steigerung des Rohholzaufkommens stellen die bisher nicht bewirtschafteten Waldflächen dar.

Auf der Basis der Clusterstudie Forst und Holz Niedersachsen lassen sich nun gemeinsame Strategien der Forst- und Holzwirtschaft entwickeln.

Stichworte: Kennzahlen Holzwirtschaft, Holzaufkommensprognose, Nutzungsverhalten der Forstbetriebe

\begin{abstract}
The globalisation of wood and wood products markets presents new challenges for both forestry and the forest products industry in Germany. It has lead to an increased demand for timber, and to the expansion of production capacity in the timber industry. In response, the Forest and Wood Cluster Study in Lower Saxony aims to enhance the competitiveness of forestry and the forest products industry. Consequently, in addition to an analysis of the inner structures (enterprise, turnover, number of employees) and economic potential of this industry, the potential timber yields were predicted for different management regimes over a period of 30 years, and the critical factors influencing the expected timber exploitation levels in these forest enterprises identified.

Lower Saxony possesses the third largest forest area of the states in Germany. The high percentage of small private forests, and the unfavourable forest age structures make it difficult to keep timber utilisation levels consistent. With a turnover of about 15 billion Euro in 2005, tax statistics indicate that the Lower Saxony forest and wood cluster contributed to $3.4 \%$ of the state's total turnover. Moreover, the turnover generated from the forest and wood cluster in Lower Saxony is still ranked the fourth highest in the country, even though a number of other federal states recently have expanded the production capacities of their sawn timber and wood based panel industries.

Various timber yield prediction scenarios for near-natural, yield, and conservation oriented forest management objectives revealed that there is little scope for modifying silvicultural strategies to increase timber exploitation in forests that are already intensively, yet well-managed without jeopardising forest sustainability. An assessment of the production potential of unmanaged forest areas provides the greatest opportunity to increase wood production levels.
\end{abstract}


The Forest and Wood Cluster Study, Lower Saxony provides a basis for the development of future joint strategies between forestry and the forest products industry.

Key words: timber industry parameters, prediction of timber harvesting potential, forest management objectives 



\section{Einleitung}

\subsection{Anlass und Ziele}

Der Forst-Holz- und Papier-Sektor umfasst alle Gewerbe, deren Hauptgeschäft auf Materialien, Dienstleistungen oder Produkten direkt oder indirekt aus dem Wald beruht (BECKEMAN u. LUUKKO 2005). Seine volkswirtschaftliche Bedeutung wurde lange Zeit in Deutschland unterschätzt. Durch die Globalisierung der Forstund Holzwirtschaft und die zunehmende Verknappung fossiler Rohstoffe hat dieser Wirtschaftszweig in den letzten Jahren einen enormen Aufschwung erfahren. Dieter und Thoroe bezifferten 2003 seinen Beitrag zum gesamtwirtschaftlichen Bruttoproduktionswert bereits auf 3,1\%. Besonders im ländlichen Raum haben die klein- und mittelständischen Betriebe dieses Clusters einen wesentlichen Einfluss auf die regionale Wirtschaftsentwicklung und Beschäftigungspolitik. Dies trifft in besonderem Maße für das Flächenland Niedersachsen $\mathrm{zu}$, für das bisher noch keine Bestandsaufnahme des Forst-Holz- und PapierSektors mit seinen Verflechtungen zu den benachbarten Bundesländern und zum Ausland vorliegt. Diese Lücke soll mit der vorliegenden Studie geschlossen werden.

Ziel der Clusterstudie Forst und Holz Niedersachsen ist es, die Wettbewerbsfähigkeit der niedersächsischen Forst- und Holzwirtschaft zu stärken. Hierzu sollen die inneren Strukturen (Betriebe, Umsätze, Beschäftigungszahlen) und wirtschaftlichen Potenziale analysiert, die möglichen Holzaufkommen bei Unterstellung verschiedener Bewirtschaftungsstrategien für einen Zeitraum von 30 Jahren prognostiziert und die wesentlichen Einflussfaktoren auf das zu erwartende Nutzungsverhalten der Forstbetriebe aufgezeigt werden. Diese Ergebnisse bilden die Grundlagen für eine gemeinsame Betrachtung sämtlicher Wirtschaftsbereiche des Forst-Holz- und Papier-Sektors, um die gegenwärtigen und künftigen Chancen und Risiken im Wettbewerb vor allem aus Sicht der Rohstoffverfügbarkeit aufzuzeigen und Strategien zur Optimierung der Wirtschaftsprozesse konzipieren zu können. Im Einzelnen sollen folgende Informationen bereitgestellt werden:

- Ermittlung der Wirtschaftsdaten (Jahresumsatz, Anzahl der Betriebe und Anzahl der Beschäftigten) für den Forst-Holz- und Papier-Sektor

- Beschreibung der wichtigsten Branchendaten und aktuellen Entwicklungen der Holz verarbeitenden und bearbeitenden Industrie

- Darstellung und Analyse der regionalen und überregionalen Ströme des niedersächsischen Rohholzes

- Analyse des Nutzungsverhaltens und der Nutzungsintensität anhand der Daten der BWI 1 und 2 für den Zeitraum 1987 - 2002; Vergleich der 
Nutzungsintensitäten der verschiedenen Eigentumsarten in Abhängigkeit von verschiedenen Bestandestypen.

- Fortschreibung der Ergebnisse der zweiten Bundeswaldinventur in Niedersachsen zum Stichtag 01.01.2007 und Berücksichtigung der Schäden durch den Orkan Kyrill in der ersten Prognoseperiode

- Abschätzung des Holzaufkommens bei Unterstellung alternativer Bewirtschaftungsstrategien („naturnah“, „ertragsorientiert“, „naturschutzorientiert") getrennt nach Baumartengruppen, Vor- und Endnutzung sowie Sortimenten

- Quantifizierung und Analyse der Differenzen zwischen den bis 2007 fortgeschriebenen und ab dann bis 2036 prognostizierten Holzvorräten und Nutzungsmöglichkeiten und den durch die BWI 2 ermittelten Holzvorräten im Jahr 2002 sowie den Ergebnissen der WEHAM-Holzaufkommensprognose der Bundesforschungsanstalt für Forst- und Holzwirtschaft, Hamburg (BFH)

- Rückkoppelung der Ergebnisse der forstlichen Produktionsplanung mit den Entwicklungen des Holzsektors

- Vorbereitung von Diskussionsgrundlagen zur Ableitung von Strategien und konkreten Handlungsempfehlungen für den Forst-Holz- und PapierSektor

Die Clusterstudie Forst und Holz Niedersachsen wurde vom Niedersächsischen Ministerium für den ländlichen Raum, Ernährung, Landwirtschaft und Verbraucherschutz finanziert und als Gemeinschaftsprojekt der Nordwestdeutschen Forstlichen Versuchsanstalt - Abteilung Waldwachstum -, der Abt. Forstökonomie u. Forsteinrichtung des Burckhardt-Instituts der Universität Göttingen und des Niedersächsischen Kompetenznetzes für Nachhaltige Holznutzung (NHN) e. V. durchgeführt. Unter der Leitung der Professoren Dr. Hermann Spellmann und Dr. Jürgen Nagel von der Nordwestdeutschen Forstlichen Versuchsanstalt (NW-FVA) wurde der Forst-Holz- und Papier-Sektor durch Ass. d. Fd. Burkhard Rüther analysiert und die Rohholzpotenziale durch M. Sc. Jan Hansen berechnet; unter Leitung von Professor Dr. Bernhard Möhring von der Abt. Forstökonomie u. Forsteinrichtung des Burckhardt-Instituts der Georg-August-Universität Göttingen und Dr. Matthias Dieter von der Bundesforschungsanstalt für Forst- und Holzwirtschaft wurde die Verfügbarkeit der Rohholzpotenziale durch Ass.'in d. Fd. Agatha Ludwig bearbeitet. Die Verbreitung der Projektergebnisse wird dem Niedersächsischen Kompetenznetz für Nachhaltige Holznutzung (NHN) e. V. in Abstimmung mit dem Niedersächsischen Ministerium für den ländlichen Raum, Ernährung, Landwirtschaft und Verbraucherschutz obliegen. 


\subsection{Hintergrundinformationen}

\subsubsection{Stellung der niedersächsischen Forstwirtschaft im nationalen Vergleich}

Nach den Ergebnissen der zweiten Bundeswaldinventur (BMVEL 2004) liegt Niedersachsen mit einem Waldflächenanteil von $24,3 \%$ unter dem bundesweiten Durchschnitt von $31 \%$. Das Maximum von $42,1 \%$ ist in Rheinland-Pfalz zu finden, den geringsten Waldanteil weist Schleswig-Holstein mit 10,3\% auf. Absolut gesehen hat das Flächenland Niedersachsen mit 1.155 .737 ha die drittgrößte Waldfläche in Deutschland. Hinsichtlich der Eigentumsverhältnisse ist Niedersachsen durch einen hohen Privatwaldanteil gekennzeichnet (59\%). Nur in Nordrhein-Westfalen ist diese Eigentumsart mit $67,0 \%$ noch stärker vertreten. Der Bundesdurchschnitt liegt bei 43,6\%. Der Landeswaldanteil entspricht mit $29 \%$ dem Bundesdurchschnitt. Der Bundeswald nimmt $5 \%$ der niedersächsischen Gesamtwaldfläche ein, der Körperschaftswald $7 \%$. Die führenden Baumarten sind Kiefer, Fichte, Buche und Eiche, deren Anteile am Holzvorrat 27\%, $23 \%$, $18 \%$ bzw. $12 \%$ betragen (vgl. Abb. 1) (NDS. MinisTERIUM F. D. LÄNDLICHEN RAUM, ERNÄHRUNG, LANDW. U. VERBRAUCHERSCHUTZ 2004).

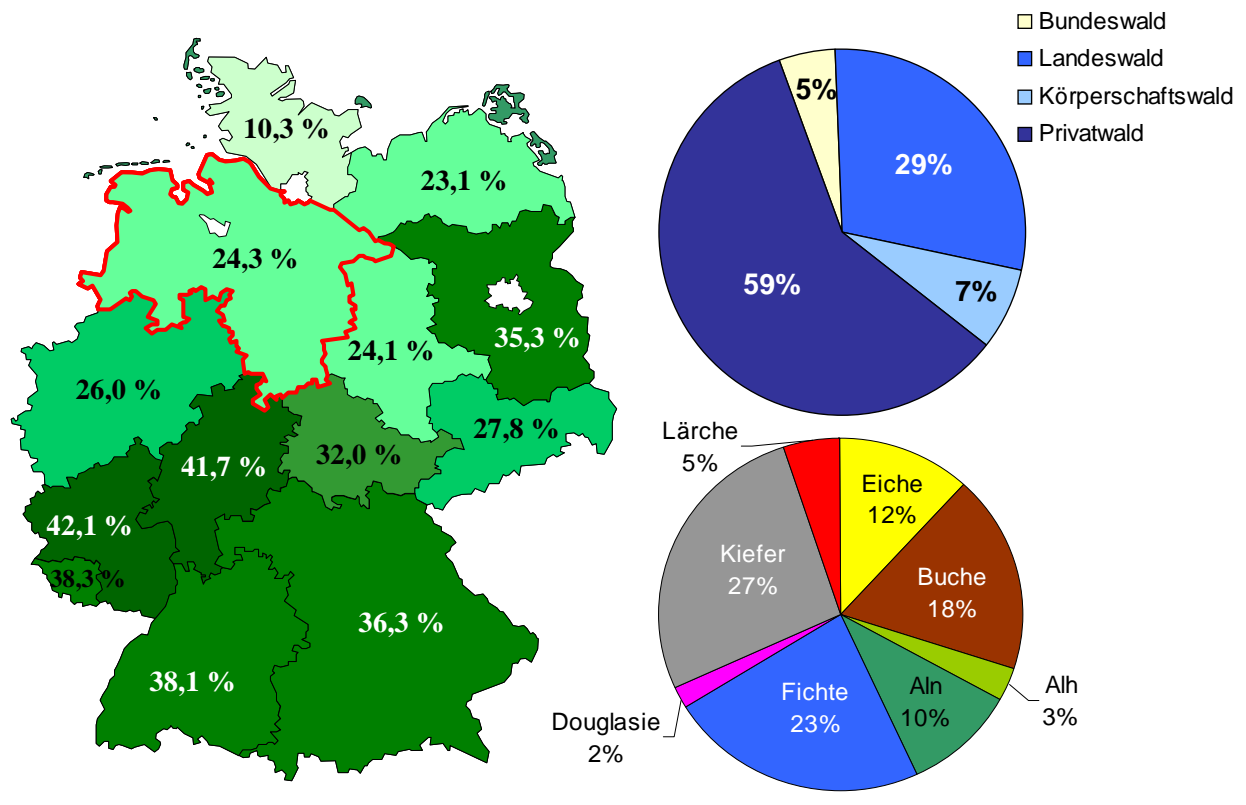

Abbildung 1: Links: Bundesländer und ibr Waldflächenanteil; rechts oben: Waldflächenanteil nach Eigentumsarten in Niedersachsen; rechts unten: Baumartenanteile am HolzvorratsGesamtvolumen in Niedersacbsen 
Der Gesamtholzvorrat in Niedersachsen beträgt ca. 296 Mio Vfm. Er setzt sich zu $43 \%$ aus Laub- und zu $57 \%$ aus Nadelholz zusammen, wobei Laubholz mit niedriger Lebensdauer (ALn) mit einem Anteil von 10\% bedeutend am Vorrat beteiligt ist. Betrachtet man die Verteilung der Baumartenflächenanteile bezogen auf die zwanzigjährigen Altersklassen (vgl. Abb. 2), zeigt sich, dass in Niedersachsen vor allem das Nadelholz und die Laubhölzer mit kurzen Umtriebszeiten in den Altersklassen II und III (21-40 bzw. 41-60 Jahre) vertreten sind. Die Anteile des ALn stocken größtenteils auf Moorstandorten und sind somit meist der forstwirtschaftlichen Nutzung entzogen. Eiche und Buche weisen insgesamt einen ausgeglichenen Altersklassenaufbau auf. In den letzten 20 Jahren sind weit unterdurchschnittlich Nadelbaumarten nachgezogen worden. Der in den letzten Jahren verstärkte Anbau der Douglasie zeigt sich in dem erhöhten Anteil dieser Baumart in den jungen Altersklassen. $80 \%$ der Douglasie sind der Altersklasse I und II zuzuordnen.

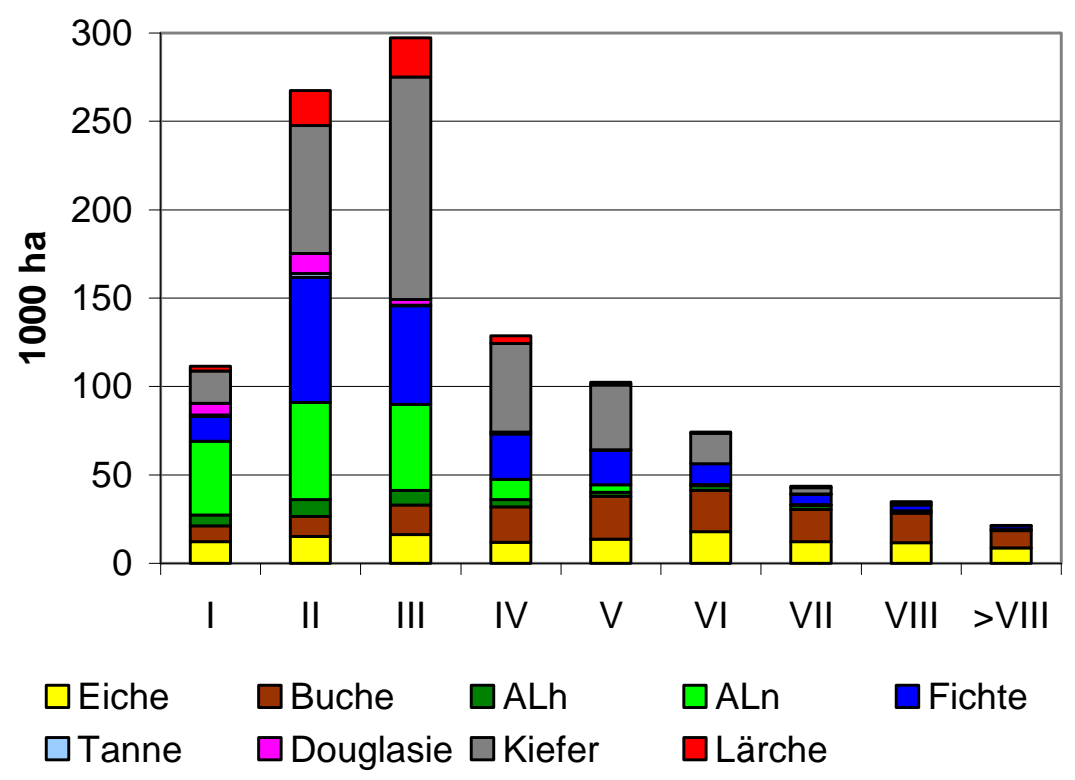

Abbildung 2: Flächenanteile der Baumartengruppen im Hauptbestand nach Altersklassen

Hinsichtlich des Zuwachses ist im Vergleich der alten Bundesländer Niedersachsen knapp hinter Hessen das Land mit dem geringsten jährlichen Zuwachs $\left(10,57 \mathrm{~m}^{3} / \mathrm{ha}^{*} \mathrm{a}\right)$. Die höchsten Zuwächse sind in Baden-Württemberg zu verzeichnen $\left(13,24 \mathrm{~m}^{3} / \mathrm{ha}^{*} \mathrm{a}\right)$. Der bundesweit durchschnittliche Zuwachs beträgt $12,12 \mathrm{~m}^{3} / \mathrm{ha}$ a. Der jährliche Holzeinschlag in Niedersachsen betrug zwischen den Jahren 1987 und 2002 4,5 Mio. Efm bzw. 4,3 Efm/ha. Dieser Wert liegt deutlich 
unter dem Bundesdurchschnitt (ca. 7 Efm/ha) (BUNDESMINISTERIUM F. VERBRAUCHERSCHUTZ, ERNÄHRUNG U. LANDWIRTSCHAFT 2004).

\subsubsection{Globalisierung des deutschen Holzmarktes}

Die Globalisierung der Rohstoff- und Warenmärkte und die zunehmende Verknappung fossiler Rohstoffe stellen die deutsche Forst- und Holzwirtschaft vor neue Herausforderungen. Sie haben zu einer steigenden Holznachfrage und zum Aufbau neuer Produktionskapazitäten der Holzindustrie geführt.

Im globalen Wettbewerb konnte sich die deutsche Holz bearbeitende Industrie erfolgreich auf den internationalen Märkten positionieren. Die deutschen Säge-, Papier- und Holzwerkstoffindustrien gehören zu den führenden Unternehmen ihrer Branchen in Europa. Entscheidend beeinflusst wurde diese Entwicklung durch die Erschließung neuer Absatzmärkte im Ausland und den Aufbau moderner Verarbeitungseinheiten in den vergangenen Jahren. Die neuen Holz bearbeitenden Betriebe mit einem jährlichen Rohstoffbedarf von teilweise über 2 Mio. $\mathrm{m}^{3}$ sind bei dem Absatz ihrer Produkte stark von den internationalen Märkten abhängig. So konnte die deutsche Nadelholzsägeindustrie innerhalb der letzten 5 Jahre ihren Exportanteil an Nadelschnittholz um 120 \% (+2,6 Mio. m³) steigern (HEIDER 2005). Insgesamt belief sich der Export in 2005 auf ca. 5 Mio. $\mathrm{m}^{3}$ Nadelschnittholz, wodurch Deutschland seine Position als Nettoschnittholzexporteur weiter verbessern konnte.

Trotz schwacher Binnennachfrage in den zurückliegenden Jahren wurden die Produktionskapazitäten von Seiten der Holz bearbeitenden Industrie aufgrund der guten Absatzlage auf den Weltmärkten und der durch die BWI 2 ermittelten gestiegenen Holzvorräte in Deutschland weiter erhöht. Die entstandene Konkurrenz hat sich durch die bundesweite Zunahme der energetischen Holznutzung und dem Nachfrageanstieg der europäischen Säge- und Holzwerkstoffindustrie nach deutschem Säge- und Industrieholz weiter verschärft.

Obwohl zusätzliche Kapazitäten durch die Holz bearbeitende Industrie aufgebaut wurden, hat sich Deutschland in den vergangenen Jahren vom Nettorohholzimporteur (1993) zum Nettorohholzexporteur (2002) gewandelt. Die Exportüberschüsse betragen beim Nadelstamm- und Industrieholz jeweils 1 Mio. $\mathrm{m}^{3}$ und beim Laubstammholz 0,7 Mio. $\mathrm{m}^{3}$. Hinzu kommt ein Nettoexport von Holzhackschnitzeln (HOELZEL 2007). Der Nadelrohholzexport ist in den Jahren von 2000 bis 2005 um über $30 \%$ auf 2,7 Mio. Fm angestiegen. 


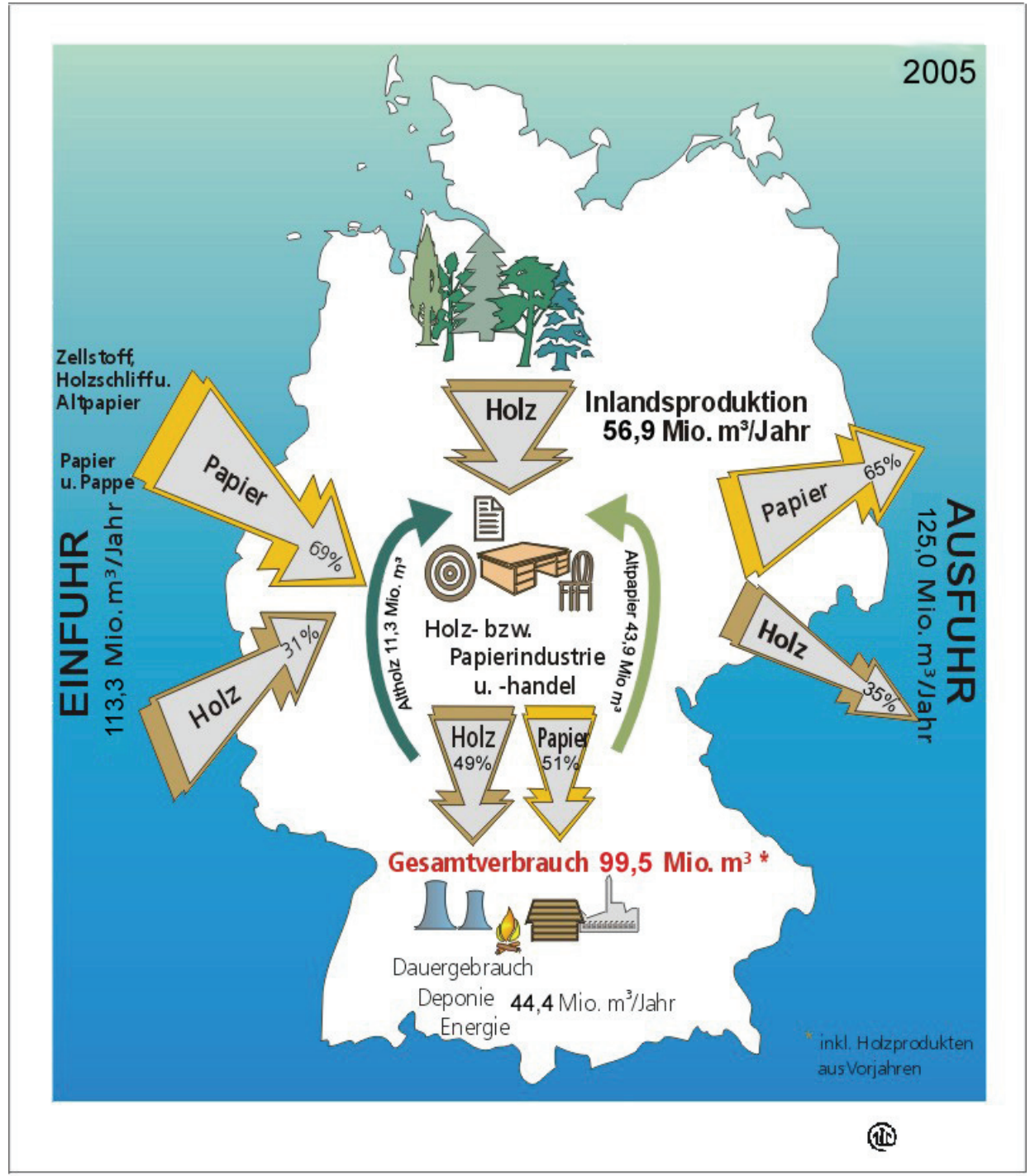

Abbildung 3: Flussdiagramm Forst- und Holzwirtschaft in der Bundesrepublik Deutschland (aktualisierte Daten: Bundesforschungsanstalt für Forst- und Holzwirtschaft, Inst. für Forstökonomie, DIETER 2007; Grafik: NFP)

Im Vergleich der Jahre 2001 und 2005 ist die Einfuhr von Holz und Papier von 101,7 Mio. $\mathrm{m}^{3}$ (2001) auf 113,3 Mio. $\mathrm{m}^{3}$ (2005) angestiegen, die Ausfuhr sogar von 92,1 Mio. auf 125 Mio. $\mathrm{m}^{3}$ und die Inlandsproduktion von Holz von 39,5 Mio. $\mathrm{m}^{3}$ auf 56,9 Mio. $\mathrm{m}^{3}$. Im gleichen Zeitraum ist der Gesamtverbrauch von Holz und Papier erst angestiegen und dann im Jahr 2005 wieder auf den Ausgangswert von 2001 gesunken (99,5 Mio. m³ s. Abb. 3). Zusätzliche Holzpotenziale liegen 
größtenteils beim Starkholz, beim Laubholz und im Kleinprivatwald. Dagegen sind im Nadelholz, in den mittleren Dimensionen und im Landeswald keine zusätzlichen Ressourcen vorhanden (HOELZEL 2007).

Es ist zu erwarten, dass durch die geplante Schaffung weiterer Produktionskapazitäten durch die Holz be- und verarbeitende Industrie die Inlandsnachfrage nach Rohholz in Deutschland weiter zunehmen wird. Ein zusätzlich ansteigender Holzbedarf von Seiten der Energiebranche würde für bestimmte Sortimente eine Erhöhung des Rohholzimports durch die Holz bearbeitende Industrie erforderlich machen, um die kontinuierliche Auslastung ihrer Produktionskapazitäten sicherstellen zu können.

Die neue Situation auf dem deutschen Holzmarkt macht die Erarbeitung einer Gesamtstrategie der Forst- und Holzwirtschaft erforderlich, insbesondere um die Wettbewerbsfähigkeit im internationalen Bereich langfristig zu sichern. 



\section{Der niedersächsische Cluster Forst und Holz}

\subsection{Allgemeines}

Dem Cluster Forst und Holz wurde in der Vergangenheit auf europäischer Ebene keine besondere Bedeutung zugemessen. Erst durch die Initiative waldreicher europäischer Mitgliedsländer erfolgte von Seiten des Europäischen Parlaments (BundesRat 2001) eine Definition des Clusters Forst und Holz. Demnach umfasst dieser Cluster neben den bisherigen Wirtschaftsbereichen der Forstwirtschaft, der holzbe- und -verarbeitenden Industrie und der Papierwirtschaft zusätzlich auch die Wirtschaftsbereiche Verlags- und Druckereiwesen (vgl. Abb. 4).
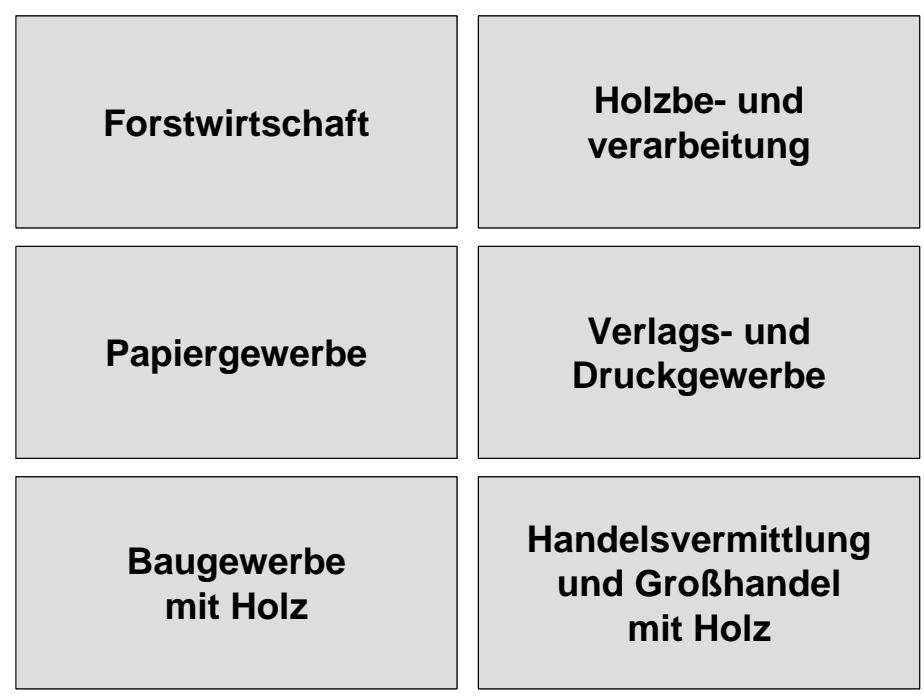

Abbildung 4: Wirtschaftsbereiche des Clusters Forst und Holz.

In Deutschland wurden basierend auf der Clusterdefinition der Europäischen Union (BUNDESRAT 2001) unterschiedliche Ansätze zur Analyse des Clusters Forst und Holz auf Bundes- und Landesebene verfolgt.

Zur volkswirtschaftlichen Beschreibung des Clusters eignen sich dabei Angaben über die Zahl der Betriebe und Beschäftigten sowie die Umsätze (DIETER u. THOROE 2003).

Von DiETER und THOROE (2003) wurde die volkswirtschaftliche Bedeutung des deutschen Clusters Forst und Holz auf Basis amtlicher Statistiken sowie Ergebnissen der Handwerkszählungen und der Handels- und Gaststättenzählung ermittelt. Ein vergleichbarer Ansatz findet sich auch bei MOSREK et. al. (2005). 
Die Erhebung der volkswirtschaftlichen Kennzahlen des niedersächsischen Clusters Forst und Holz erfolgte auf Grundlage der Umsatzsteuer- und Beschäftigungsstatistik des Statistischen Landesamtes Niedersachsen. Ein ähnlicher Ansatz wurde auch von SEEgMÜLLER (2005) für die Darstellung des Forst-Holz- und Papier-Sektors in Rheinland-Pfalz verwendet.

Die Zulieferbranche des Forst- und Holz-Bereichs (u. a. Hersteller von Holzbearbeitungsmaschinen und Klebstoffindustrie) sowie die Branche der energetischen Nutzung konnten bei der Erhebung der volkswirtschaftlichen Kennzahlen des Clusters Forst und Holz nicht berücksichtigt werden, da die notwendigen Daten auf Basis der Umsatzsteuer- und Beschäftigungsstatistik nicht ermittelt werden konnten.

\subsection{Material und Methoden}

\subsubsection{Erhebung von Umsatz, und Unternehmensanzahl}

Die Umsatzsteuerstatistik gilt als zuverlässig, weil sie keine Stichprobe ist, sondern mit Hilfe von Finanzangaben die Grundgesamtheit aller umsatzsteuerpflichtigen Unternehmen erfasst (ANONYMUS 1997).

Grundlage dieser Statistik ist die Besteuerung der Umsätze von Unternehmen, die in tiefer regionaler und sektoraler Gliederung von dem Statistischen Landesamt ermittelt werden. Es werden alle umsatzsteuerpflichtigen Unternehmen erfasst, die im jeweiligen Berichtsjahr Umsatzsteuer-Voranmeldungen in Deutschland abgegeben haben und deren Jahresumsatz im Berichtsjahr mindestens 17.500 Euro beträgt ( $(19$ Abs. 1 UStG).

Im Gegensatz zu den Branchenstatistiken, der Handels- und Gaststättenzählung und Handwerkszählung basiert die Umsatzsteuerstatistik nicht auf einer Betriebs- sondern auf einer Unternehmensauswertung (SEEGMÜLLER 2005).

Ein Unternehmen wird in der amtlichen Statistik als kleinste rechtlich selbstständige Einheit definiert, die aus handels- bzw. steuerrechtlichen Gründen Bücher führt und eine jährliche Feststellung des Vermögensbestandes bzw. des Erfolgs der wirtschaftlichen Tätigkeit vornehmen muss. Das Unternehmen umfasst alle zugehörigen Betriebe. Ein Unternehmen kann somit mehrere Betriebe umfassen. Ein Betrieb ist eine Niederlassung an einem bestimmten Ort. Zu dem Betrieb zählen zusätzlich örtlich und organisatorisch angegliederte Betriebsteile. Wenn ein niedersächsisches Unternehmen Betriebe außerhalb des Landes unterhält, würden die Umsätze dieser Betriebe trotzdem in der niedersächsischen Umsatzstatistik aufgeführt. Dieses hat jedoch in der Forst- und Holzwirtschaft kaum Bedeutung (DIETER et. al. 2004; WEBER 2001). Die Einteilung der Wirtschaftszweige (vgl. Tab. 1) erfolgte entsprechend den Ausführungen des europäischen Parlaments unter Berücksichtigung der Ergänzungen von DIETER u. THOROE (2003) und 
SEEGMÜLLER (2005) sowie der Beachtung der „Klassifikation der Wirtschaftsqweige“ (STATISTISCHES BUNDESAMT 2003).

Tabelle 1: Untergliederung des Clusters Forst und Holz unter Beachtung der „Klassifizierung der Wirtschaftsqweige "(STATISTISCHES BUNDESAMT 2003)

\section{Forstwirtschaft}

Forstwirtschaft (o. Erbringung von Dienstleistungen)

Erbringung von forstlichen Dienstleistungen

\section{Holzbe- und -verarbeitung}

Holzgewerbe

Säge-, Hobel- und Holzimprägnierwerke

Herstellung von Furnier-, Sperrholz, Holzfaser-und Holzspanplatten

Herstellung von Konstruktionsteilen, Fertigbauteilen, Ausbauelementen u. Fertigteilbauten aus Holz

Herstellung von Verpackungsmitteln, Lagerbehältern und Ladungsträgern aus Holz

Herstellung von Holzwaren, anderweitig nicht genannt (ohne Herstellung von Möbeln)

Herstellung von Möbeln, Schmuck, Musikinstrumenten, Sportgeräten, Spielwaren u. sonstigen Erzeugnissen

Herstellung von Sitzmöbeln

Herstellung von Büro- und Ladenmöbeln

Herstellung von Küchenmöbeln

Herstellung von sonstigen Möbeln

\section{Papier-, Verlags- und Druckgewerbe}

Papiergewerbe

Herstellung von Holz- und Zellstoff, Papier, Karton u. Pappe

Herstellung von Waren aus Papier, Karton u. Pappe

\section{Verlagsgewerbe}

Verlegen von Büchern

Verlegen von Zeitungen

Verlegen von Zeitschriften

\section{Druckgewerbe}

\section{Baugewerbe mit Holz}

Bautischlerei und -schlosserei

Zimmerei und Ingenieurholzbau

Parkettlegerei

\section{Handelsvermittlung und Großhandel mit Holz}

Handelsvermittlung von Holz, Baustoffen und Anstrichmitteln

Großhandel mit Roh- und Schnittholz

Großhandel mit sonstigen Holzwaren sowie Bauelementen aus Holz

Beiträge aus der NW-FVA, Band 1, 2007 
Die Darstellung der Umsatzentwicklung der Abteilungen des Clusters Forst und Holz erfolgte für den Zeitraum von 1998-2005, da innerhalb dieses Zeitraums keine Veränderung bei der „Klassifizierung der Wirtschaftszweige" vorgenommen wurde.

\subsubsection{Erbebung der Beschäftigten}

Die Beschäftigten des Clusters Forst und Holz wurden mit Hilfe der Beschäftigungsstatistiken des Statistischen Landesamtes Niedersachsen ermittelt. Zu den sozialversicherungspflichtig Beschäftigten zählen Arbeitnehmer, die kranken-, renten- und arbeitslosenversicherungspflichtig sind oder für die von den Arbeitgebern Beitragsanteile zur gesetzlichen Rentenversicherung entrichtet werden. Angaben über Betriebe mit sozialversicherungspflichtig Beschäftigten werden von der Bundesagentur für Arbeit jährlich übermittelt.

In den gelieferten Daten sind diejenigen Betriebe enthalten, in denen zum Stichtag 31.12. sozialversicherungspflichtig Beschäftigte tätig waren. Darüber hinaus sind Angaben zu solchen Betrieben enthalten, in welchen zwar zum Stichtag keine, jedoch mindestens in einem der übrigen Quartals-Stichtage sozialversicherungspflichtig Beschäftigte arbeiteten.

\subsubsection{Volkswirtschaftliche Gesamtrechnung}

Für die Wirtschaftsbereiche des Clusters Forst und Holz wurden nach dem Europäischen System Volkswirtschaftlicher Gesamtrechnungen (ESVG 1995) einige wichtige Kennzahlen aus der Entstehungs- und Verteilungsrechnung der Volkswirtschaftlichen Gesamtrechung (VGR) zusammengestellt. Das Europäische System Volkswirtschaftlicher Gesamtrechnungen ist eine europaweit einheitliche Methodik für die gemeinsamen Normen, Definitionen, Klassifizierungen und Verbuchungsregeln, die die Erstellung von Konten und Tabellen der Volkswirtschaftlichen Gesamtrechnung auf vergleichbaren Grundlagen ermöglicht (STATISTISCHES BUNDESAMT 2003). Sie liefert im Nachhinein einen quantitativen Überblick über das wirtschaftliche Geschehen der Volkswirtschaft.

Die für die Erstellung der VGR benötigten Daten wurden vom Statistischen Landesamt Niedersachsen zur Verfügung gestellt. (Die Autoren bedanken sich bei Herrn Andreas Jacobs für die Bereitstellung.) Die Berechnung der VGR erfolgte nach dem Inlandskonzept. So wurden alle wirtschaftlichen Aktivitäten des Clusters Forst und Holz in Niedersachsen unabhängig vom Wohnort der Erwerbstätigen erfasst. Die VGR wurde für die Wirtschaftsabteilungen „Forstwirtschaft“, „Holzgewerbe“, „Papiergewerbe“, „Verlags- und Druckgewerbe“ und „Herstellung von Möbeln, Schmuck, Musikinstrumenten, Sportgeräten, Spielwaren und sonstigen Erzeugnissen“ erstellt. Für die Wirtschaftsabteilungen „Baugewerbe“ und 
„Handelsvermittlung und Großhandel“" lagen keine Daten vor. Entsprechend konnten sie bei der Erstellung der VGR nicht berücksichtigt werden.

\subsubsection{Sonstige Quellen}

Mit einer schriftlichen Befragung der Niedersächsischen Forstdienststellen (Nds. Landesforsten, Landwirtschaftskammer Niedersachsen, Bundesforstämter, Klosterforsten, kommunale, städtische und private Forstbetriebe) wurden die Absatzwege des niedersächsischen Rohholzes bis zur ersten Verarbeitungsstufe erfasst. Die niedersächsischen Holzverkaufsmengen des Jahres 2005 konnten getrennt nach sägefähigem bzw. nicht-sägefähigem Laub- und Nadelholz dem jeweiligen Zielbundesland bzw. Ausland zugeordnet werden. Zusätzlich wurde der Anteil des Holzhandels an der ersten Verarbeitungsstufe ermittelt, um den Weiterverkauf des unbearbeiteten Rohholzes einschätzen zu können.

\subsection{Ergebnisse}

\subsubsection{Entwicklung der Jahresumsätže}

Der niedersächsische Cluster Forst und Holz hatte 2005 einen Jahresumsatz von ca. 15 Mrd. €. Damit steht Niedersachsen im Ländervergleich an vierter Stelle nach Nordrhein-Westfalen, Baden-Württemberg und Bayern. Wird der Umsatz auf die Einwohnerzahl des Bundeslandes bezogen, rückt Niedersachsen in der Rangfolge sogar noch weiter nach oben.

Trotz des Aufbaus neuer Produktionskapazitäten der Säge- und Holzwerkstoffindustrie in zahlreichen Bundesländern in den vergangenen Jahren konnte Niedersachsen seine Stellung im bundesweiten Vergleich behaupten.

Am gesamten steuerbaren Umsatz des Landes Niedersachsen von ca. 440 Mrd. $€$ im Jahr 2005 hatte der Cluster Forst und Holz einen Anteil von ca. 3,4 \%. Durch den niedersächsischen Cluster Forst und Holz wurde in der Zeit von 1998-2005, trotz der zeitweisen Konjunkturschwäche, ein durchschnittlicher Jahresumsatz von 14-15 Mrd. € erwirtschaftet.

Zum Vergleich: Der Umsatz des bundesdeutschen Clusters Forst und Holz liegt bei ca. $100 \mathrm{Mrd} € €$ (DIETER u. THOROE 2003) und der jährliche Umsatz des europäischen Clusters bei 550-600 Mrd. € (BECKEMAN u. LUUKKO 2005).

Von den Wirtschaftsabteilungen des Clusters Forst und Holz hatte die Abteilung Verlags- und Druckgewerbe mit 4,3 Mrd. € den höchsten Jahresumsatz. Dieser entspricht einem Anteil von $30 \%$ am Gesamtumsatz des Clusters (vgl. Abb. 5). Weiterhin gehören das Papiergewerbe mit 3,8 Mrd. € (25\%) und die Holzbe- und -verarbeitung mit 3,4 Mrd. € (23\%) zu den umsatzstärksten Abtei- 
lungen des Clusters. Von den drei Abteilungen werden annährend $80 \%$ des Gesamtumsatzes des niedersächsischen Clusters Forst und Holz erwirtschaftet.

Die Forstwirtschaft hat, trotz ihrer besonderen Bedeutung als Rohstoffproduzent innerhalb der Wertschöpfungskette, mit 270 Mio. $€$ den geringsten Anteil am Gesamtumsatz. Ähnliche Ergebnisse zeigen auch eine bundesweite Studie (Diether u. ThOroe 2003; MOsReK et. al. 2005) bzw. Clusterstudien anderer Bundesländer (SEEGMÜLLER 2005).

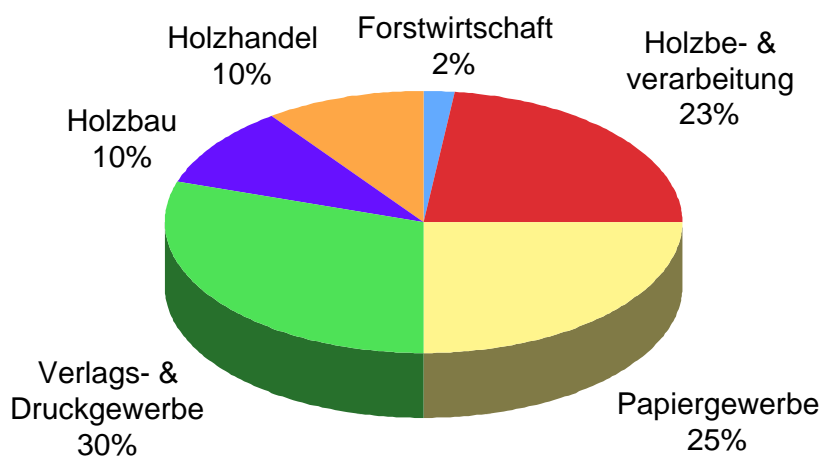

Abbildung 5: Prozentualer Anteil der Wirtschaftsbereiche am Gesamtumsatz des Clusters Forst und Holz in Niedersachsen im Jahr 2005 (STATISTISCHES LANDESAMT NiEDERSACHSEN 2006)

Von 1998-2002 war das Papiergewerbe mit durchschnittlich 4,5 Mrd. € die umsatzstärkste Abteilung des niedersächsischen Clusters, fiel aber 2003 durch einen deutlichen Umsatzrückgang hinter das Verlags- und Druckgewerbe zurück (vgl. Abb. 6). Der Rückgang von 1,4 Mrd. € in der Wirtschaftsgruppe „Herstellung von Holzstoff, Zellstoff, Papier, Pappe und Karton“ ist auf strukturelle Veränderungen innerhalb der Branche zurückzuführen. Wie bereits SEEGMÜLLER (2005) für Rheinland-Pfalz nachweisen konnte, ist auch in Niedersachsen das Papiergewerbe, im Gegensatz zu den anderen Abteilungen des Clusters, über die Landesgrenzen hinaus unternehmerisch besonders stark vernetzt. Da es sich bei dieser Wirtschaftsgruppe in der Regel um Großunternehmen handelt, sind unternehmerische Veränderungen bzw. Umstrukturierungen meist mit einem deutlichen Umsatzanstieg bzw. -rückgang verbunden. 
Das klein- und mittelständisch geprägte Verlags- und Druckgewerbe ist im hohen Maße von der Werbewirtschaft und den Werbeeinnahmen abhängig. Trotz der bundesweiten schwachen Konjunktur der letzten Jahre konnte diese Abteilung ihren Umsatz kontinuierlich steigern. Mit ca. $4 \mathrm{Mrd}$. $€$ hatte das niedersächsische Verlags- und Druckgewerbe einen Anteil von $7 \%$ am bundesweiten Gesamtumsatz der Branche von 58 Mrd. € (MOSREK et. al. 2005).

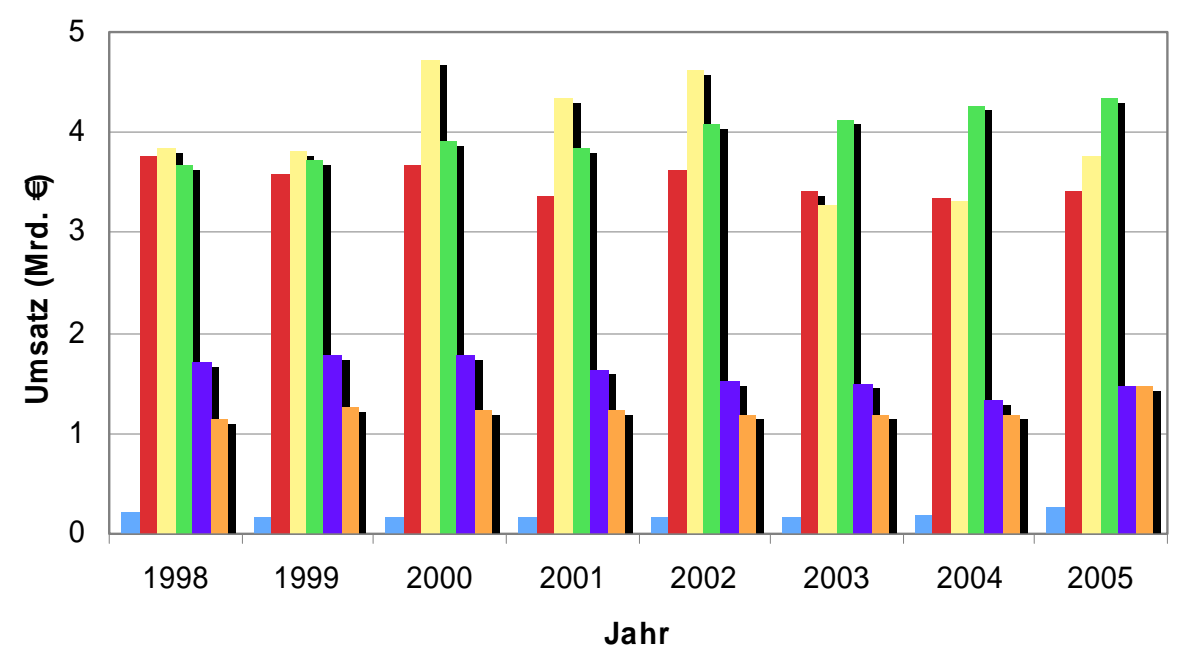

\footnotetext{
Forstwirtschaft

Holzbe- \& verarbeitung

Papiergewerbe

Verlags- \& Druckgewerbe $\square$ Holzbau

-Holzhandel
}

Abbildung 6: Umsätze der Wirtschaftsbereiche des niedersächsischen Clusters Forst und Holz. (STATISTISCHES LANDESAMT NIEDERSACHSEN 2006)

Der niedersächsische Holzbau konnte nach den Umsatzrückgängen zwischen 2001-2004 im Jahr 2005 erstmals wieder ein Umsatzplus verzeichnen (vgl. Abb. 6). Für die Zukunft bieten sich der Branche insbesondere durch die Modernisierung und den Ausbau bestehender Gebäude neue Perspektiven. Mitverantwortlich für diesen Trend ist die Energieeinsparverordnung (EnEV), die Hausbesitzer zwingt, ihre Immobilien effektiv vor Heizwärmeverlust zu schützen. Außerdem verstärkt sich bei den Neubauten der Trend zum Holzhaus. Zwar liegt der Anteil in Deutschland mit 15 Prozent noch relativ niedrig im Vergleich zu anderen europäischen Ländern, aber die Tendenz ist deutlich zu spüren (BUND DEUTSCHER ZIMMERMEISTER 2006).

Der deutliche Umsatzanstieg der forstlichen Produktion im Jahr 2005 um über $40 \%$ ist nicht durch die Erhöhung des Holzeinschlages oder durch Preiserhöhung des Rohholzes, sondern im Wesentlichen durch die Umstrukturierung der Niedersächsischen Landesforstverwaltung entstanden. Da die neu gegründete Anstalt des 
öffentlichen Rechts „Niedersächsische Landesforsten“ seit 2005 für die Regelbesteuerung bei der Umsatzsteuer optiert hat, wurde in diesem Jahr auch erstmals der Umsatz der Anstalt in der offiziellen Statistik ausgewiesen. Die forstwirtschaftlichen Betriebe, die entsprechend des Umsatzsteuergesetzes pauschalieren, werden über die Umsatzsteuerstatistik des Landes Niedersachsens nicht erfasst. Eigene Erhebungen haben gezeigt, dass dies keinen bedeutenden Einfluss auf das Gesamtergebnis des Clusters Forst und Holz hat.

Die Holzbe- und -verarbeitung besteht aus den Wirtschaftsabteilungen „Holzgewerbe“ und „Herstellung von Möbeln, Schmuck, Musikinstrumenten, Sportgeräten, Spielwaren und sonstigen Erzeugnissen“.

Zum Holzgewerbe gehören die Sägeindustrie (Säge-, Hobel- u. Holzimprägnierwerke), Holzwerkstoffindustrie (Furnier-, Sperrholz-, Holzfaser- und Holzspanplatten), die Herstellung von Holzbauteilen (Hersteller von Konstruktionsbauteilen, Fertigbauteilen, Ausbauelementen u. Fertigbauteilen aus Holz), die Verpackungsindustrie (Hersteller von Verpackungsmitteln, Lagerbehältern und Ladungsträgern aus Holz) und die Holzwarenhersteller (Herstellung von Holzwaren, anderweitig nicht genannt (ohne Herstellung von Möbeln)).

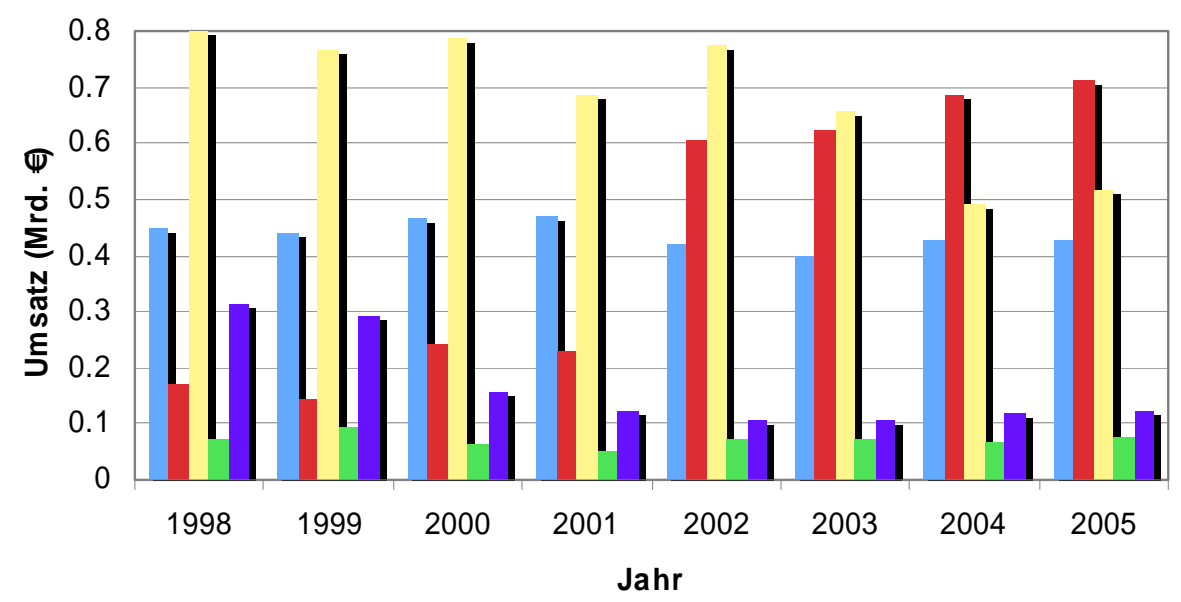

Sägeindustrie $\quad$ Holzwerkstoffindustrie
Herstellung von Holzbauteilen $\square$ Verpackungsindustrie
Holzwarenherstellung

Abbildung 7: Jäbrlicher Umsatz des niedersäcbsischen Holggewerbes (STATISTISCHES LANDESAMT NIEDERSACHSEN 2006) 
Mit ca. 700 Mio. $€$ Umsatz im Jahr 2005 ist die Holzwerkstoffindustrie die umsatzstärkste Branche des Holzgewerbes. Der deutliche Umsatzanstieg dieser Wirtschaftsgruppe im Jahr 2002 ist auf die Inbetriebnahme einer neuen Produktionseinheit eines niedersächsischen Holzwerkstoffherstellers zurückzuführen. Obwohl sich das neue Werk außerhalb Niedersachsens befindet, wird der Umsatz, bedingt durch den niedersächsischen Unternehmenssitz, in der Umsatzsteuerstatistik des Landes Niedersachsens ausgewiesen (s. Kap 2.3.2).

Die niedersächsische Holzwerkstoffindustrie produzierte 2005 ca. $120.000 \mathrm{~m}^{3}$ Spanplatten und $625.000 \mathrm{~m}^{3}$ Faserplatten (SÖRGEL u. MANTAU 2006b). Dies entspricht einem Anteil von 6\% an der deutschen Span- und Faserplattenproduktion.

Der Umsatz aus der Herstellung von Holzbauteilen ist durch die schwache Baukonjunktur seit dem Jahr 2000 um ca. 300 Mio. € zurückgegangen. Im Jahr 2005 konnten die niedersächsischen Hersteller von Holzbauteilen erstmals wieder eine Umsatzerhöhung erzielen. Die niedersächsische Sägeindustrie hatte von 19982005 einen jährlichen Umsatz von ca. 400-450 Mio. €.

\subsubsection{Entwicklung der Unternehmenszablen}

Mit rund 10.000 Unternehmen ist der Cluster Forst und Holz ein von klein- und mittelständischen Unternehmen geprägter Wirtschaftssektor. Die Wirtschaftsabteilung Holzbau hat mit 4.100 die meisten Unternehmen des Clusters Forst und Holz (vgl. Abb. 8). Mit 2.600 und 1.800 Unternehmen folgen die Abteilungen „Holzbe- und -verarbeitung“ und „Verlags- und Druckgewerbe“.

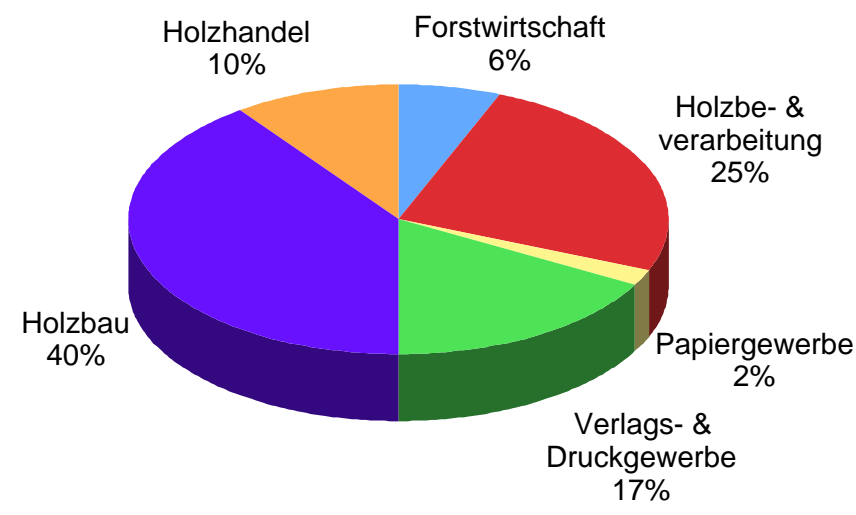

Abbildung 8: Prozentuale Verteilung der Unternebmen des niedersächsischen Clusters Forst und Holz. 
Die Unternehmen des niedersächsischen Clusters Forst und Holz haben durchschnittlich 8 Beschäftigte. Das Papiergewerbe hat mit $2 \%$ (vgl. Abb. 8) den geringsten Unternehmensanteil im Forst- und Holz-Bereich. Es besteht zum überwiegenden Teil aus Großunternehmen und besitzt mit durchschnittlich 75 Beschäftigten je Unternehmen einen wesentlich höheren unternehmensbezogenen Beschäftigtenanteil als die anderen Wirtschaftsabteilungen des Clusters. Ein Blick auf die Entwicklung der Unternehmensanzahl des Clusters Forst und Holz zeigt, dass die Anzahl der Unternehmen in den Jahren 2002-2004 deutlich gegenüber den Vorjahren zurückgegangen ist.

Die konjunkturelle Schwächeperiode im Baubereich hat auch im niedersächsischen Cluster Forst und Holz zu zahlreichen Unternehmensschließungen geführt. In der Sägeindustrie wurden von den 213 niedersächsischen Sägewerken im Jahr 2001 (MANTAU u. SÖRGEL 2004) bis zum Jahr 2005 über 50 Werke (SÖRGEL u. MANTAU 2006a) stillgelegt. Neben der strukturellen Veränderung zu großen Verarbeitungseinheiten, insbesondere im Bereich der Nadelholzsägeindustrie, haben auch Betriebsaufgaben aufgrund fehlender Zukunftsperspektiven im Zuge des Generationswechsels zu dieser Entwicklung geführt.

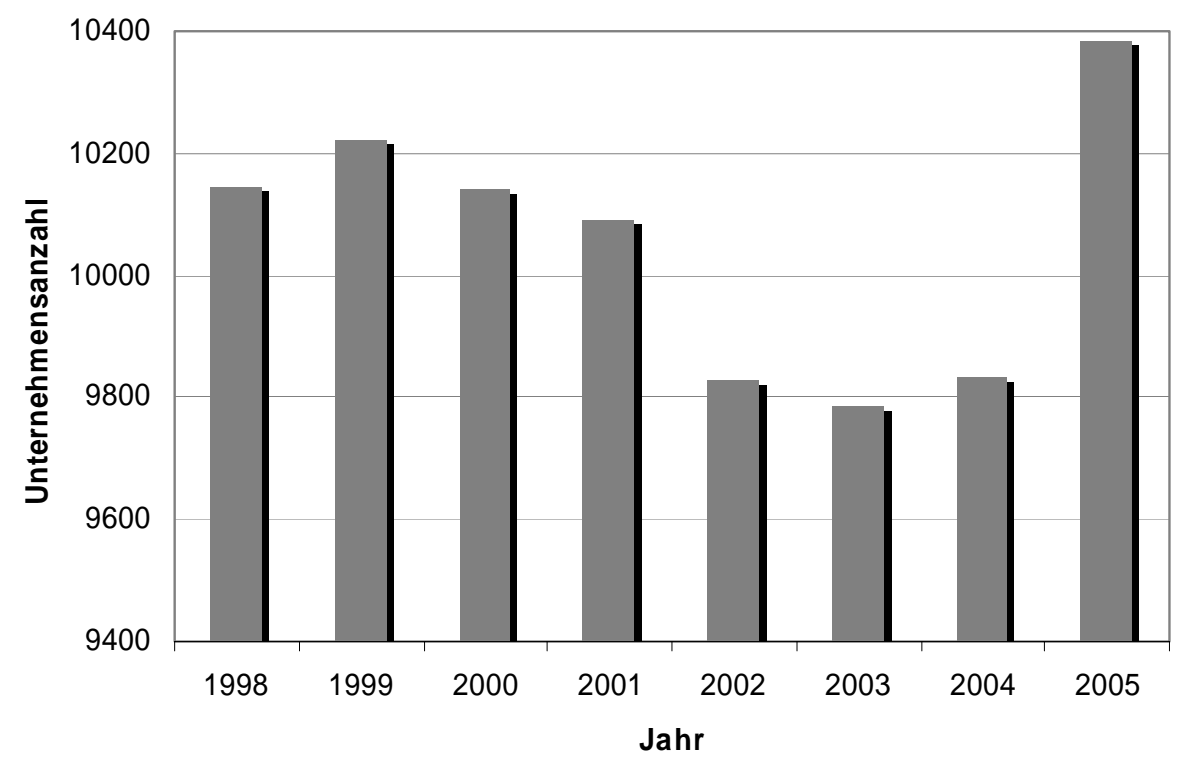

Abbildung 9: Entwicklung der Unternehmensanzabl des niedersächsischen Clusters Forst und Holz. (STATISTISCHES LANDESAMT NIEDERSACHSEN 2006)

Im Jahr 2005 kam es durch die Neugründung von ca. 300 Unternehmen in der Wirtschaftsabteilung „Holzbau“ und ca. 100 Unternehmen in der Wirtschaftsgruppe „Herstellung von Konstruktionsbauteilen, Fertigbauteilen, Ausbauelemen- 
ten und Fertigbauten aus Holz" zu einer deutlichen Erhöhung der Unternehmensanzahl im Cluster Forst und Holz (vgl. Abb. 9). Ein Grund für die Zunahme der Unternehmen der deutschen Zimmereibranche liegt in der Altgesellenregelung von 2004, nach der sich bestimmte Gesellen auch ohne Meisterprüfung selbstständig machen dürfen. Diese Regelung setzte eine Gründungsdynamik in Gang, die auch 2005 noch anhielt (BUND DEUTSCHER ZIMMERMEISTER 2006).

Bei einem Vergleich der Unternehmensanzahl der Wirtschaftzweige Niedersachsens zeigt sich, dass die Unternehmen des Clusters Forst und Holz einen Anteil von ca. $4 \%$ an den ca. 260.000 Unternehmen des Landes Niedersachsen haben. Die meisten Unternehmen haben die Wirtschaftszweige „Grundstücks- und Wohnungswesen, Vermietungen, Dienstleistungen F.U.“ mit 68.000 und „Handel, Instandhaltung und Reparatur von KFZ und Gebrauchsgütern“ mit 67.000 Unternehmen.

Der Cluster Forst und Holz ist geprägt durch die hohe Anzahl der klein- und mittelständischen Unternehmen entlang der Wertschöpfungskette. Besonders im ländlichen Raum Niedersachsens bieten die Unternehmen des Clusters Forst und Holz vielfältige Beschäftigungsmöglichkeiten.

\subsubsection{Entwicklung der Beschäftigtenzablen}

In Niedersachsen haben 2006 ca. 77.000 sozialversicherungspflichtige Beschäftigte im Cluster Forst und Holz Arbeit gefunden (vgl. Abb. 11). Da Unternehmer und Beamte aus dem Forst- und Holz-Bereich sowie die Zulieferbranche nicht berücksichtigt wurden, ist von einer höheren Anzahl Erwerbstätiger im niedersächsischen Cluster Forst und Holz auszugehen.

An den ca. 2,3 Mio. sozialversicherungspflichtig Beschäftigten des Landes Niedersachsens haben die Beschäftigten des Clusters Forst und Holz einen Anteil von ca. $3 \%$ (STATISTISCHES LANDESAMT NIEDERSACHSEN 2006).

Vergleichsweise stellt der Cluster Forst und Holz in Thüringen ca. 40.000 (THÜRINGER MINISTERIUM FÜR LANDWIRTSCHAFT, NATURSCHUTZ UND UMWELT 2007), in Rheinland-Pfalz ca. 50.000 (SEEGMÜLLER 2005), in Bayern ca. 185.000 (BAYER. STAATSMINISTERIUM F. LANDWIRTSCHAFT U. FORSTEN 2006) und bundesweit ca. 915.364 Arbeitsplätze (DIETER u. THOROE 2003). Bei der Ermittlung der Beschäftigungszahlen wurden neben der Beschäftigungsstatistik teilweise auch andere Statistiken berücksichtigt, weshalb die Studien nur bedingt vergleichbar sind.

Das Verlags- und Druckgewerbe stellt mit rund 22.700 sozialversicherungspflichtigen Beschäftigten und einem Anteil von 29\% die meisten Arbeitsplätze im Cluster Forst und Holz (vgl. Abb. 10 und 11). Die Abteilung Holzbe- und -verarbeitung ist mit 20.500 und einem Beschäftigungsanteil von ca. $27 \%$ der zweitwichtigste Arbeitgeber des niedersächsischen Clusters. 


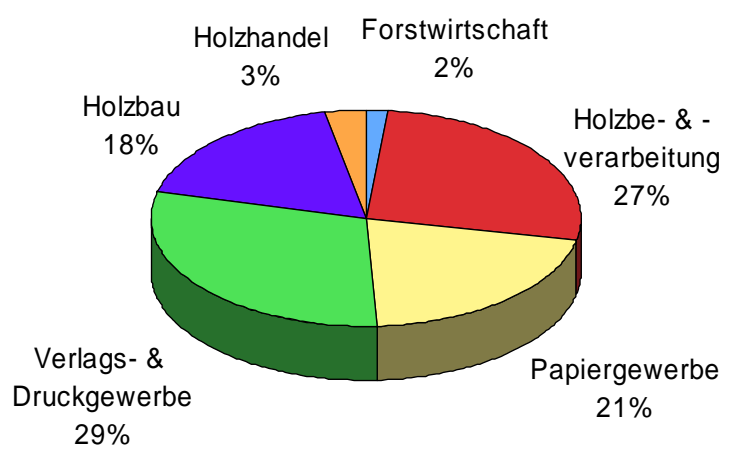

Abbildung 10: Prozentualer Anteil der sozialversicherungspflichtig Beschäftigten des Clusters Forst und Holz im Jahr 2006 (STATISTISCHES LANDESAMT NIEDERSACHSEN 2006)

Die Anzahl der Beschäftigten im niedersächsischen Cluster Forst und Holz ist in den vergangenen Jahren deutlich zurückgegangen. Seit 1998 sind mit ca. 26.000 mehr als $25 \%$ der gesamten sozialversicherungspflichtigen Arbeitsplätze des Clusters Forst und Holz weggefallen (vgl. Abb. 11). Neben der bereits angesprochenen Konjunkturschwäche haben auch strukturelle Veränderungen innerhalb der Abteilungen des Clusters diesen starken Rückgang ausgelöst.

Abbildung 11 stellt diesen Stellenrückgang in der Zeit von 1998 bis 2006 aufgeschlüsselt nach den einzelnen Wirtschaftsabteilungen des Clusters Forst und Holz dar. Der Stellenabbau zeichnete sich in der Holzbe- und -verarbeitung am deutlichsten ab. In den letzen acht Jahren ist in der Abteilung fast jeder dritte Arbeitsplatz weggefallen. Auch im Verlags- und Druckgewerbe haben Re- und Umstrukturierungsmaßnahmen zum Abbau von fast 6.000 Arbeitsplätzen geführt; ebenso wie beim „Holzbau“. Besonders bei den klein- und mittelständischen Unternehmen ist in den vergangenen Jahren der Beschäftigungsanteil zurückgegangen, wohingegen es in der niedersächsischen Papierindustrie nur zu einem Abbau von $2 \%$ der Beschäftigten kam.

Ein Blick auf die Wirtschaftsgruppe „Holzgewerbe“ der Abteilung (vgl. Abb. 12) zeigt, dass insbesondere bei der „Herstellung von Holzbauteilen“ mehr als $40 \%$ der Arbeitsplätze abgebaut wurden.

Auch in der Sägeindustrie ist die Beschäftigtenzahl von 2.100 Mitarbeitern im Jahr 1998 auf 1.500 Mitarbeiter im Jahr 2006 gesunken. Der Rückgang der Beschäftigten resultiert im Wesentlichen aus der bereits angesprochenen strukturellen Veränderung innerhalb der Branche. Es ist davon auszugehen, dass sich dieser Prozess auch in Zukunft weiter fortsetzen wird. 


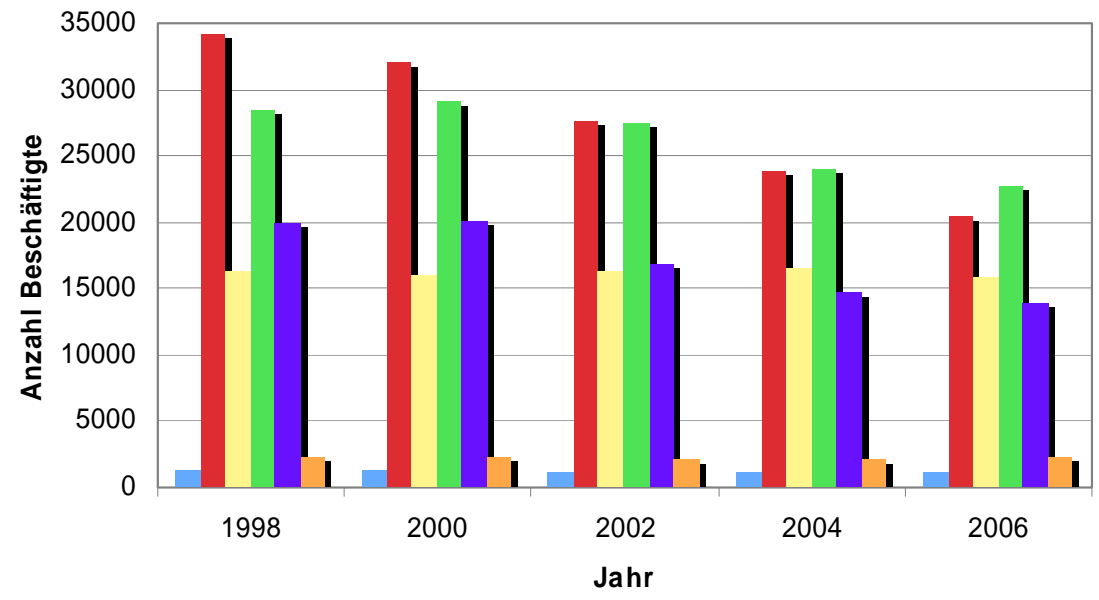
Forstwirtschaft
- Holzbe- \& -verarbeitung
Papiergewerbe
- Verlags- \& Druckgewerbe Holzbau
- Holzhandel

Abbildung 11: Entwicklung der Beschäftigtenzablen der Abteilungen des Clusters Forst und Holz von 1998-2006 (STATISTISCHES LANDESAMT NiEDERSACHSEN 2006)

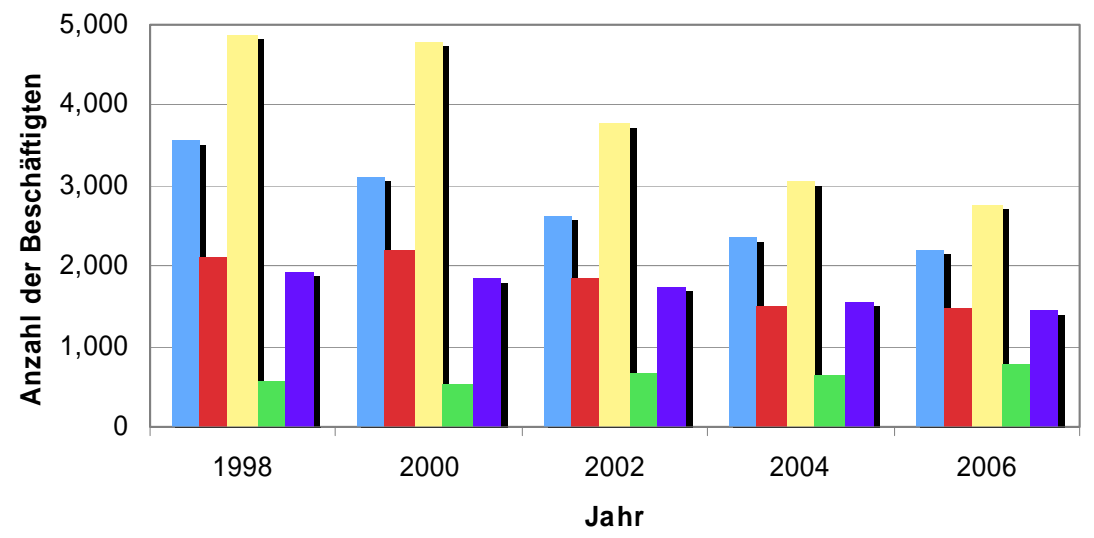

$$
\begin{aligned}
& \text { Sägeindustrie } \quad \text { Holzwerkstoffindustrie } \\
& \text { Herstellung von Holzbauteilen } \square \text { Verpackungsindustrie } \\
& \text { Holzwarenherstellung }
\end{aligned}
$$

Abbildung 12: Entwicklung der Beschäftigungszablen des Holzgewerbes von 1998-2006 (STATISTISCHES LANDESAMT NIEDERSACHSEN 2006) 
In der Holzwerkstoffindustrie kam es durch die Stilllegung eines Spanplattenbetriebes in Südniedersachsen im Jahr 2002 zu einem Rückgang der Beschäftigung.

Die Verpackungsindustrie kann als einzige Wirtschaftsgruppe des Holzgewerbes seit 2003 eine kontinuierliche Erhöhung ihrer Beschäftigtenzahl vorweisen. Entscheidend hierfür sind der anhaltende Exportboom und die jüngste Konjunkturbelebung im Inland (ANONYMUS 2007).

Der niedersächsische Möbelsektor gehört mit einem Umsatz von ca. 1,6 Mrd. € zu den wirtschaftsstärksten Abteilungen des Clusters Forst und Holz. Die stattgefundenen technischen Weiterentwicklungen und Rationalisierungsprozesse innerhalb der Branche haben den Wegfall von über 9.000 Arbeitsplätzen von 1998 bis 2006 hervorgerufen (vgl. Abb. 13).

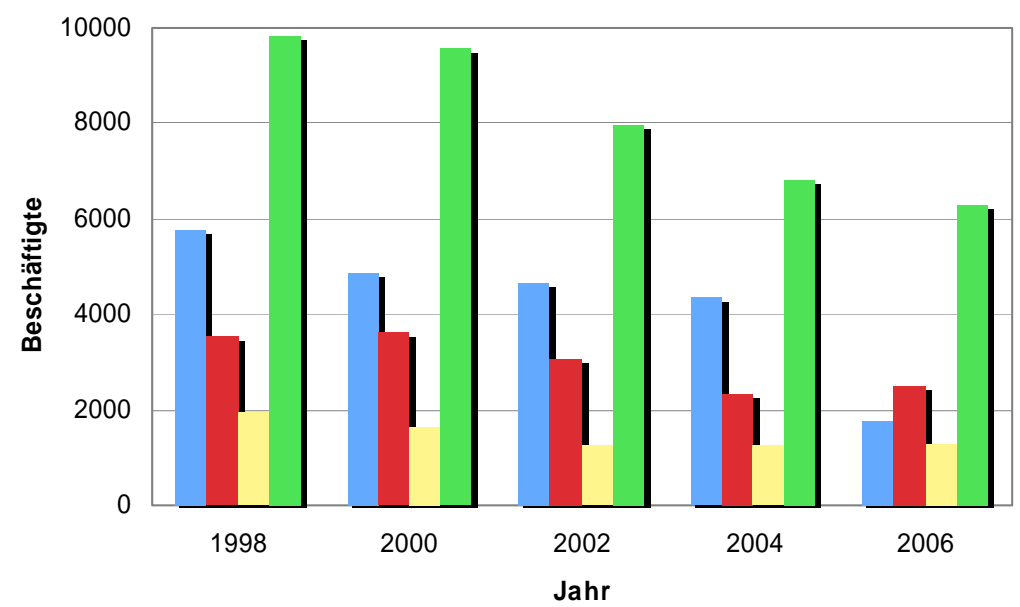

Sitzmöbel —Büro- \& Ladenmöbel Küchenmöbel —Sonstige Möbel

Abbildung 13: Entwicklung der Beschäftigungszablen der Möbelhersteller von 1998-2006 (STATISTISCHES LANDESAMT NIEDERSACHSEN 2006)

Das entspricht einem Rückgang von über $40 \%$, womit die Möbelbranche am deutlichsten vom Beschäftigungsrückgang im Forst- und Holz-Bereich betroffen ist. Die durchschnittliche Beschäftigungszahl je Unternehmen ist in der Branche um $50 \%$ auf 16 Mitarbeiter gesunken.

Die meisten Arbeitsplätze wurden in den Wirtschaftsgruppen „Herstellung von Sitzmöbeln“ mit ca. 4000 Mitarbeitern und der „Herstellung von sonstigen Möbeln" mit über 3.500 abgebaut. Neben den bereits genannten Ursachen haben auch die Verlagerung von Produktionsstandorten in Richtung Osteuropa und der zunehmende Import von „Billigmöbeln“ aus dem Ausland diese Entwicklung unterstützt. 


\subsubsection{Die volkswirtschaftliche Gesamtrechnung}

Die Erstellung wichtiger volkswirtschaftlicher Kennzahlen des niedersächsischen Clusters Forst und Holz erfolgte entsprechend den Vorgaben des „Europäischen Systems volkswirtschaftlicher Gesamtrechnungen“. Da zu den Abteilungen „Baugewerbe mit Holz“ und „Holzhandel“ keine Informationen vorlagen, konnten sie bei der Berechnung nicht berücksichtigt werden. Auch von SEEGMÜLLER (2005) und DiETER u. THOROE (2003) wurden diese Abteilungen nicht berücksichtigt.

Von dem niedersächsischen Cluster Forst und Holz wurde im Jahr 2004 ein Bruttoproduktionswert von ca. $10 \mathrm{Mrd}$ € erwirtschaftet (vgl. Tab. 2). Dieser entspricht einem Anteil von 2,9\% an dem gesamtwirtschaftlichen Bruttoproduktionswert Niedersachsens. Damit liegt der niedersächsische Cluster Forst und Holz auf dem gleichen Niveau wie der von DiETER u. THOrOE (2003) ermittelte bundesweite Vergleichswert.

Das Papiergewerbe hat mit fast $40 \%$ den höchsten Anteil am Bruttoproduktionswert. Das Druck- und Verlagsgewerbe, das im bundesweiten Cluster Forst und Holz den höchsten Anteil ausmacht, liegt in Niedersachsen bei einem Anteil von ca. $32 \%$. Dem Holzgewerbe kommt in Niedersachsen mit $22 \%$ eine geringere Bedeutung zu als auf Bundesebene oder im Bundesland Rheinland-Pfalz (SEEGMÜLLER 2005). Der Anteil der Forstwirtschaft liegt mit ca. $3 \%$, wie im Bund, deutlich hinter dem Bruttoproduktionswert der anderen Wirtschaftszweige.

Die Vorleistungen des Clusters Forst und Holz lagen im Jahr 2004 bei ca. 6,3 Mrd. €. Damit liegt der Anteil der Vorleistungen, gleich dem bundesweiten Wert, bei ca. $60 \%$. Das Papiergewerbe weist insbesondere aufgrund seiner internationalen Verflechtungen mit ca. 2,6 Mrd. € den höchsten Vorleistungsanteil der niedersächsischen Forst- und Holz-Branche auf.

Im Jahr 2004 betrug die Bruttowertschöpfung des Clusters Forst und Holz ca. 3,7 Mrd. $€$ (vgl. Tab. 2). Der Forst- und Holz-Bereich hatte damit einen Anteil von $2,2 \%$ an der gesamtwirtschaftlichen Bruttowertschöpfung des Landes Niedersachsens. Auf Bundesebene kommen DIETER und THOROE (2003) auf einen etwas höheren Anteil von 2,5\%.

Aufgrund der höheren Vorleistungen kommt das Papiergewerbe trotz des höchsten Bruttoproduktionswertes der Wirtschaftszweige nur auf eine Bruttowertschöpfung von ca. 1,2 Mrd. €. Die größte Wertschöpfung wurde vom Verlags- und Druckgewerbe mit ca. 1,4 Mrd. € erzeugt. Mit ca. $70 \%$ stellen Papier-, Verlagsund Druckgewerbe den größten Anteil der Bruttowertschöpfung des Clusters Forst und Holz. 
Tabelle 2: $\quad$ Bruttoproduktionswerte, Vorleistungen und Bruttowertschöpfung des Clusters Forst und Holz in Niedersachsen (STATISTISCHES LANDESAMT NIEDERSACHSEN 2006)

\begin{tabular}{l|c|c|c}
\hline Wirtschaftszweig & $\begin{array}{c}\text { Brutto- } \\
\text { produktionswert } \\
\text { (Mio. } € \text { ) }\end{array}$ & $\begin{array}{c}\text { Vorleistungen } \\
\text { (Mio. } € \text { ) }\end{array}$ & $\begin{array}{c}\text { Bruttowert- } \\
\text { schöpfung } \\
\text { (Mio. } € \text { ) }\end{array}$ \\
\hline $\begin{array}{l}\text { Forstwirtschaft } \\
\text { Holzgewerbe }\end{array}$ & 267 & 102 & 164 \\
$\begin{array}{l}\text { Papiergewerbe } \\
\text { Verlags- u. }\end{array}$ & 3.779 & 903 & 415 \\
$\begin{array}{l}\text { Druckgewerbe } \\
\text { Herstellung von } \\
\text { Möbeln etc. }\end{array}$ & 3.246 & 1.827 & 1.194 \\
\hline $\begin{array}{l}\text { Summe } \\
\text { Anteil an der } \\
\begin{array}{l}\text { Gesamtwirtschaft } \\
(\%)\end{array}\end{array}$ & 1.441 & 891 & 550 \\
\hline Niedersachsen & $\mathbf{1 0 . 0 5 2}$ & $\mathbf{6 . 3 0 8}$ & $\mathbf{3 . 7 4 2}$ \\
\hline
\end{tabular}

In Niedersachsen wurden im Jahr 2004 insgesamt ca. 100 Mrd. $€$ Einkommen an 3,2 Mio. Arbeitnehmer ausbezahlt. Damit haben die Arbeitnehmerentgelte einen Anteil von ca. $60 \%$ an der gesamten Bruttowertschöpfung des Landes Niedersachsen (vgl. Tab. 3).

Der niedersächsische Cluster Forst und Holz stellt ca. 2,6 Mrd. € Einkommen für die 79.900 Arbeitnehmer des Forst- und Holz-Bereichs zur Verfügung, was einem Anteil von 2,6\% an der niedersächsischen Gesamtwirtschaft entspricht. Der Anteil der Arbeitnehmerentgelte von ca. $70 \%$ an der Bruttowertschöpfung zeigt, dass die Arbeitsintensität der Forst- und Holz-Branche über dem Landesdurchschnitt liegt.

Der Durchschnittsverdienst eines niedersächsischen Arbeitnehmers lag im Jahr 2004 bei ca. $31.200 €$. Im verarbeitenden Gewerbe lag der durchschnittliche Verdienst mit $42.200 €$ deutlich über dem Landesdurchschnitt. Die Arbeitnehmer im Cluster Forst und Holz kamen durchschnittlich auf ein Einkommen von $32.800 €$. Die Arbeitnehmer des rheinland-pfälzischen Clusters Forst und Holz hatten im Jahr 2002 einen Durchschnittsverdienst von 35.000,- € (SEEGMÜLLER 2005). 
Tabelle 3: $\quad$ Arbeitnehmerentgelte und Arbeitnehmerzablen des niedersächsischen Clusters Forst und Holz im Jahr 2004 (STATISTISCHES LANDESAMT NIEDERSACHSEN 2006)

\begin{tabular}{l|c|c}
\hline Wirtschaftszweig & $\begin{array}{c}\text { Arbeitnehmer- } \\
\text { entgelte } \\
\text { (Mio. } € \text { ) }\end{array}$ & $\begin{array}{c}\text { Arbeit- } \\
\text { nehmer } \\
\text { (Anzahl) }\end{array}$ \\
\hline Forstwirtschaft & 72 & 2200 \\
Holzgewerbe & 338 & 10.200 \\
Papiergewerbe & 817 & 19.400 \\
Verlags- u. Druckgewerbe & 883 & 33.600 \\
Herstellung von Möbeln etc. & 507 & 14.500 \\
\hline Summe & 2.617 & $\mathbf{7 9 . 9 0 0}$ \\
\hline Anteil an der Gesamtwirtschaft (\%) & $2,6 \%$ & $2,5 \%$ \\
\hline Niedersachsen & 99.706 & 3.170 .100 \\
\hline
\end{tabular}

Die höchsten Arbeitnehmerentgelte im niedersächsischen Cluster Forst und Holz im Jahr 2004 wurden mit $42.200 €$ im Papiergewerbe bezahlt. Damit erreichen die Arbeitnehmer das durchschnittliche Gehaltsniveau im verarbeitenden Gewerbe und liegen fast $30 \%$ über dem Durchschnittsgehalt der Forst- und Holz-Branche.

Die niedrigsten Entgelte bezahlten das Verlags- und Druckgewerbe mit durchschnittlich $26.300 €$. Sie liegen damit $20 \%$ unter dem Durchschnittsgehalt des Forst- und Holz-Bereichs.

Die niedersächsische Forstwirtschaft bezahlte 2004 ihren Arbeitnehmern ein Durchschnittsgehalt von $32.700 €$. Die Forstbranche erreicht damit fast das Durchschnittsgehalt des Clusters Forst und Holz. Im Vergleich mit RheinlandPfalz liegt das durchschnittliche Einkommen der niedersächsischen Forstleute ca. $20 \%$ höher.

\subsection{Darstellung der (über-)regionalen Rohholzströme Niedersachsens}

\subsubsection{Allgemeines}

Über die Absatzwege des niedersächsischen Holzes und die Bedeutung für die inländische bzw. die Holzindustrie der benachbarten Bundesländer liegen bisher nur wenige Erkenntnisse vor.

Innerhalb der Studie erfolgte eine schriftliche Befragung der niedersächsischen Forstdienststellen. Getrennt nach sägefähigem bzw. nicht-sägefähigem Laub- und Nadelholz sollten die Forstdienstellen unter Angabe des Zielbundeslandes ihre 
Verkaufsmengen des Jahres 2005 zuordnen. Zusätzlich sollte von ihnen der Anteil des Holzhandels am Rohholzverkauf angegeben werden.

Mit Hilfe der Befragung konnten die Absatzwege von ca. 3,5 Mio. Fm des niedersächsischen Holzeinschlages des Jahres 2005 erfasst werden. Diese Menge entspricht einem Anteil von fast $80 \%$ des niedersächsischen Gesamtholzeinschlags von 4,5 Mio. Fm des Jahres 2005 (ZMP 2006).

Nachfolgend wurde das laut öffentlicher Statistik in 2005 eingeschlagene sägefähige und nicht-sägefähige Laub- und Nadelholz Niedersachsens unter Beachtung der durch die Umfrage ermittelten Absatzstruktur den Bundesländern zugeordnet.

Bei der Datenerhebung zeigte sich, dass insbesondere beim Laubholz die Ausweisung des Holzhandels durch die Kombination von Sägewerk und Handel bei zahlreichen Betrieben nur eingeschränkt möglich war. Die Ausweisung des Holzhandelsanteils wurde daher bei allen Sortimenten auf das Land Niedersachsen beschränkt, da in den anderen Bundesländern der Anteil unter $5 \%$ der vermarkteten Menge lag.

Nach SÖRGEL u. MANTAU 2006a wurden im Jahr 2004 in Niedersachsen ca. 2,2 Mio. Fm Rohholz eingeschnitten. Mit über 80 \% wurde der größte Teil von der niedersächsischen Nadelholzsägeindustrie verarbeitet. Am bundesdeutschen Gesamteinschnitt hatte Niedersachsen im Jahr 2004 einen Anteil von ca. $8 \%$.

\subsubsection{Sägefähiges Nadelholz.}

Im Jahr 2005 wurden in Niedersachsen ca. 2,3 Mio. Fm sägefähiges Nadelholz eingeschlagen (ZMP 2006). Mit fast $50 \%$ wurde der größte Teil an die niedersächsische Holz bearbeitende Industrie geliefert. Die Hauptabnehmer waren die 71 Nadelholzsägewerke und 50 Mischsägewerke mit einem jährlichen Einschnitt von ca. 1,8 Mio. Fm Nadelholz (SÖRGEL u. MANTAU 2006a).

Das größte niedersächsische Nadelholzsägewerk, mit einer jährlichen Einschnittkapazität von ca. 1,5 Mio. Fm nach der geplanten Produktionserweiterung (Herbst 2007), befindet sich in Adelebsen in Südniedersachsen. Das zweitgrößte Nadelholzsägewerk Niedersachsens ist in Bergen-Eversen im nördlichen Teil des Bundeslandes mit einer jährlichen Verarbeitungskapazität von ca. 400.000 Fm angesiedelt.

Die niedersächsische Forstwirtschaft ist nicht nur für die Nadelholzsägewerke des eigenen Bundeslandes sondern auch für die Nadelholzsägeindustrie benachbarter Bundesländer ein wichtiger Rohstofflieferant (s. Abb. 14). An die Holz bearbeitende Industrie des Landes Mecklenburg-Vorpommern wurden im Jahr 2005 fast $20 \%$ des niedersächsischen Nadelsägeholzes verkauft. Bedeutendster Abnehmer dürfte das moderne Sägewerk in Wismar mit einer jährlichen Verarbeitungskapazität von über 2 Mio. Fm sein. Zusätzlich wurden jeweils ca. $5 \%$ in die Nachbarländer Hessen und Nordrhein-Westfalen geliefert. 
Der niedersächsische Holzhandel und forstliche Selbstwerber kauften ca. $12 \%$ des sägefähigen Nadelholzes.

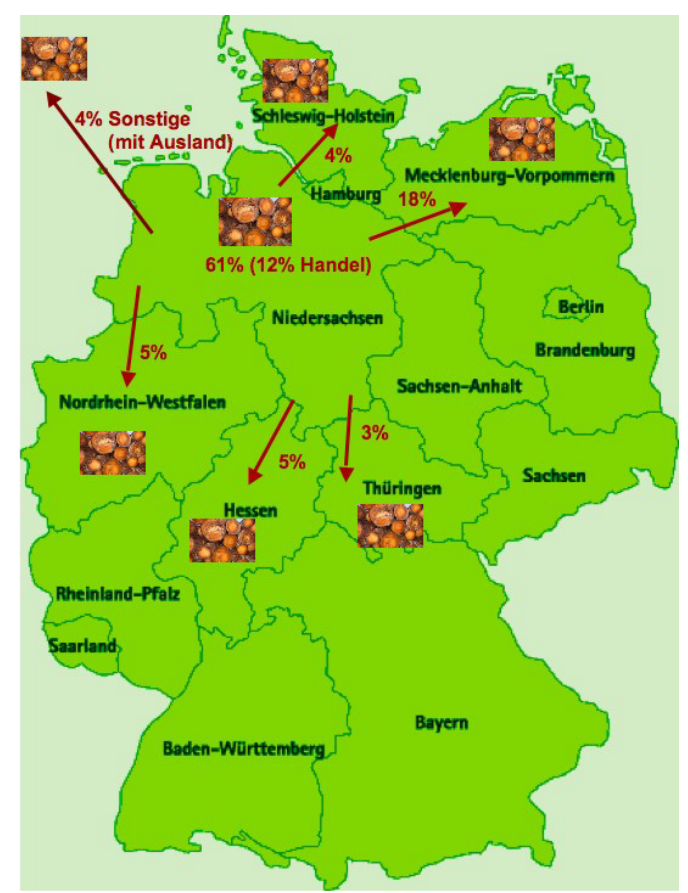

Abbildung 14: Absatzinege des sägefähigen Nadelholzes (ca. 2,3 Mio. Fm, 2005)

Der Nadelstammholzeinschlag der benachbarten Bundesländer (Brandenburg, Hessen, Mecklenburg-Vorpommern, Nordrhein-Westfalen, Sachsen-Anhalt, Schleswig-Holstein u. Thüringen) betrug im Jahr 2004 laut öffentlicher Einschlagsstatistik ca. 9,6 Mio. Fm (ZMP 2005). Im selben Jahr wurde von der Sägeindustrie dieser Bundesländer mit ca. 9,5 Mio. Fm Nadelrohholz die gleiche Menge eingeschnitten.

Bis Ende 2008 ist neben der schon erwähnten Kapazitätserweiterung eines Sägewerkes in Südniedersachsen (ca. 800.000 Fm) im Herbst 2007 auch der Neubau von drei Nadelholzsägewerken in Hessen, Nordrhein-Westfalen und SachsenAnhalt geplant, was zu einer weiteren Kapazitätserhöhung um jährlich ca. 2 Mio. Fm führen wird. Der Holzbedarf der Nadelsägeindustrie in Nordwestdeutschland wird sich dadurch zukünftig um ca. 2,8 Mio. Fm/Jahr erhöhen. 


\subsubsection{Nicht sägefähiges Nadelbolz.}

Im Jahr 2005 wurden laut öffentlicher Statistik in Niedersachsen ca. 1,7 Mio. Fm Nadelindustrieholz eingeschlagen. Hauptabnehmer waren die Holzwerkstoff- und Zellstoffindustrie sowie die Energiebranche. Insbesondere die neu entstandenen oder erweiterten Holzwerkstoff- und Zellstoffwerke in den benachbarten Bundesländern sind wichtige Abnehmer für das niedersächsische Nadelindustrieholz.

Von den $64 \%$ innerhalb Niedersachsens vermarkteten Nadelindustrieholzes wurde mit $45 \%$ der größte Anteil an den Holzhandel verkauft. Zum Handel wurden auch forstliche Selbstwerber gezählt, die neben der Holzernte auch den Transport und die Weitervermarktung übernehmen. An die niedersächsische Holz bearbeitende Industrie sowie den Energiebereich wurden ca. 19 \% Nadelindustrieholz verkauft (vgl. Abb. 15).

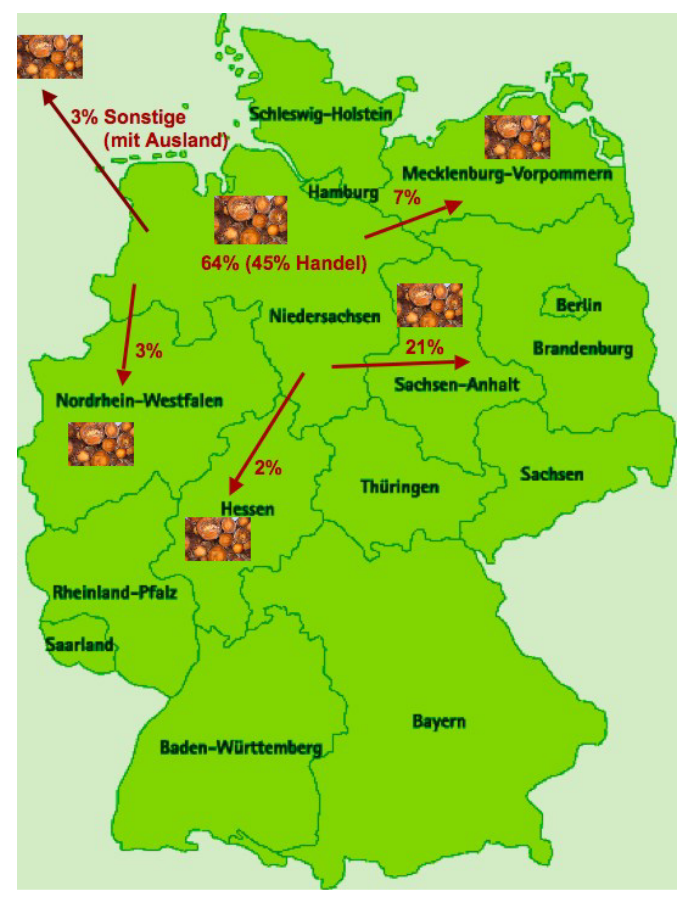

Abbildung 15: Absatzwege des nicht sägefähigen Nadelholzes (2005) 
Die Holz bearbeitende Industrie Sachsen-Anhalts kaufte 2005 über $21 \%$ Nadelindustrieholz direkt von niedersächsischen Waldbesitzern. Die Hauptabnehmer sind die neu entstandenen oder erweiterten Produktionsstätten der sachsen-anhaltinischen Holzwerkstoff- und Zellstoffproduktion. Zweitwichtigster Direktabnehmer außerhalb Niedersachsens ist mit ca. $7 \%$ die Holz bearbeitende Industrie des Landes Mecklenburg-Vorpommern.

\subsubsection{Sägefähiges Laubholz.}

Der niedersächsische Gesamtholzeinschlag des Laubsägeholzes betrug im Jahr 2005 ca. $510.000 \mathrm{Fm}$ (ZMP 2006). Das Buchenstammholz bildete mit fast 350.000 Fm den größten Anteil. Von dem eingeschlagenen Laubsägeholz wurde ca. $55 \%$ innerhalb Niedersachsens vermarktet. Der Holzhandel hatte daran einen Anteil von ca. $18 \%$.

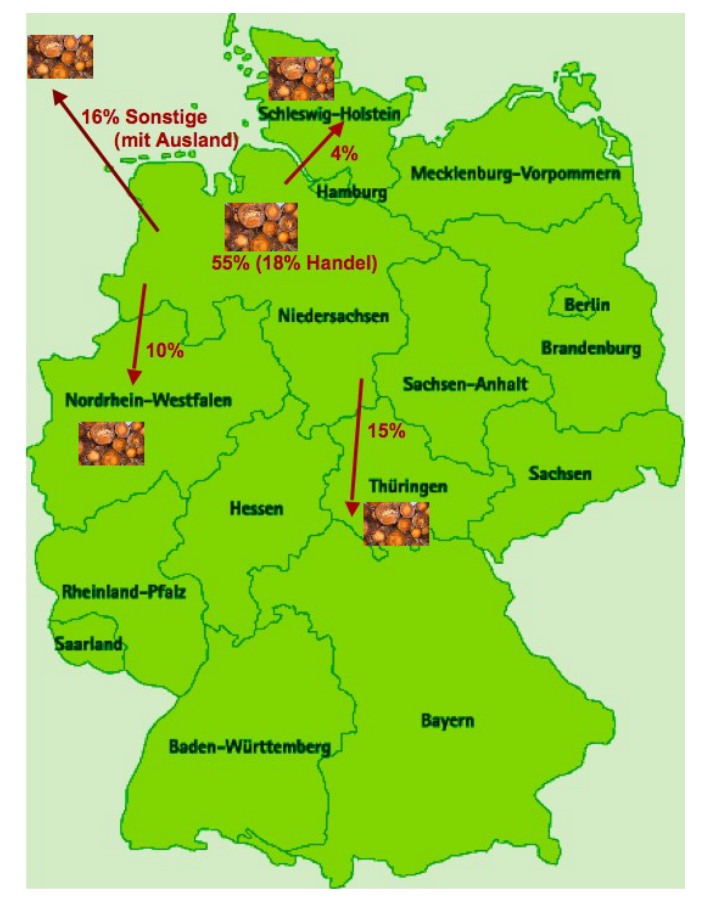

Abbildung 16: Absatzwege des sägefähigen Laubholzes (2005)

Weitere bedeutende Abnehmer für das in niedersächsischen Wäldern produzierte Laubstammholz sind die thüringische Sägeindustrie mit ca. $15 \%$ und die nordrhein-westfälische Sägeindustrie mit ca. 10\%. Bezogen auf den von SÖRGEL u. MANTAU (2006a) ermittelten niedersächsischen Gesamtlaubholzeinschnitt von ca. 
340.000 Fm im Jahr 2004 werden ca. $40 \%$ durch die niedersächsische Forstwirtschaft direkt geliefert (s. Abb. 16).

Die 41 Laubholzsägewerke und 50 Mischsägebetriebe haben einem Anteil von ca. $13 \%$ am gesamten bundesweiten Laubholzeinschnitt.

Das größte Laubholzsägewerk des Bundeslandes befindet sich in Südniedersachsen. Mit einer Einschnittskapazität von ca. $40.000 \mathrm{Fm}$ gehört es zu den größten Laubholzsägewerken im Bundesgebiet.

\subsubsection{Nicht sägefäbiges Laubhol:}

Im Jahr 2005 wurden laut öffentlicher Statistik 620.000 Fm nicht sägefähiges Laubholz in niedersächsischen Wäldern geerntet. Der größte Anteil der eingeschlagenen Rohholzmenge wurde mit ca. $87 \%$ innerhalb Niedersachsens verkauft. Laut der Umfrage wurden davon ca. $17 \%$ an den niedersächsischen Holzhandel abgesetzt. Bedeutendster Abnehmer außerhalb Niedersachsens war die Holz bearbeitende Industrie Nordrhein-Westfalens (s. Abb. 17).

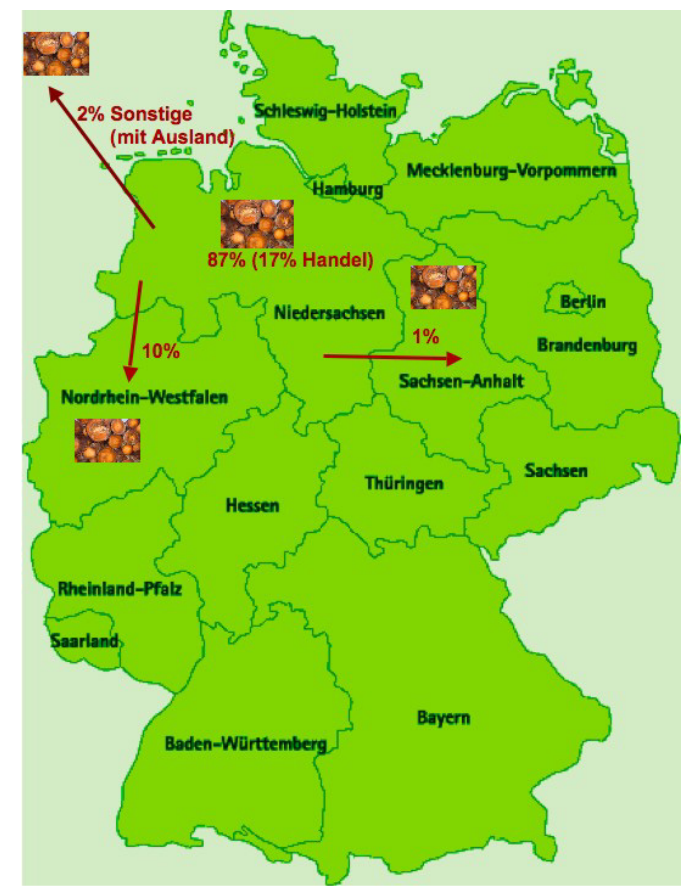

Abbildung 17: Absatzwege des nicht sägefähigen Laubholzes (2005) 
Innerhalb Niedersachsens wurde der wesentliche Teil des nicht sägefähigen Laubholzes auf dem Brennholzmarkt abgesetzt. Die Aufarbeitung erfolgte sowohl durch Forstunternehmer als auch direkt durch die Endverbraucher. Es ist davon auszugehen, dass die Nachfrage nach Holz als Energieträger in Zukunft noch weiter ansteigen wird.

\subsection{Bedeutung der energetischen Holznutzung in Niedersachsen}

Der Preisanstieg für fossile Brennstoffe hat die energetische Nutzung von Holz in den Blickpunkt der Öffentlichkeit gerückt und in den vergangenen Jahren einen deutlichen Nachfrageanstieg auf dem Energieholzmarkt ausgelöst. Es zeigt sich, dass der Bestand an Holz verbrennenden Öfen und Zentralheizungen in Niedersachsen stark angestiegen ist (vgl. Tab. 4). Entsprechend hat der Verbrauch von Brennholzsortimenten (vgl. Abb. 18) in Niedersachsen weiter zugenommen.

Bildeten in der Vergangenheit die mittleren und großen Energieanlagen aufgrund unterschiedlicher Fördermaßnahmen den Schwerpunkt, ist besonders in den vergangenen zwei Jahren die Nachfrage von privaten Verbrauchern gewachsen. Im Jahr 2006 wurden in Niedersachsen ca. 1,93 Mio. Fm Holz (Scheitholz, Pellets u. Hackschnitzel) zur Wärmeerzeugung verbraucht. Das Scheitholz ist mit ca. 1,75 Mio. Fm das bedeutendste Brennholzsortiment Niedersachsens (s. Abb. 18). Auch im gesamten Bundesgebiet hat das Scheitholz mit ca. 17 Mio. Fm (80 \%) den größten Brennstoffanteil (MANTAU u. SÖRGEL 2006).

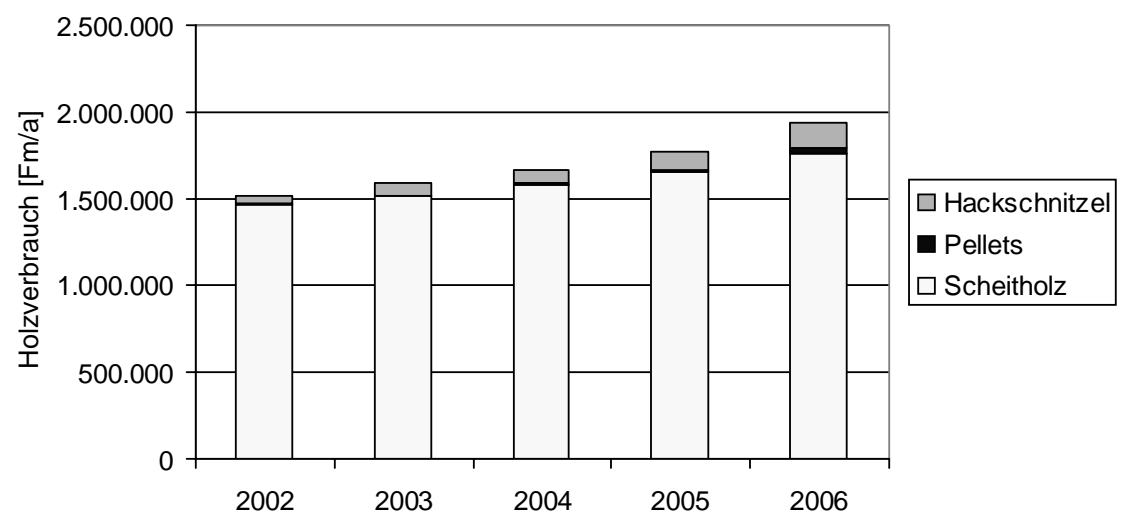

Abbildung 18: Holzverbrauch zur Wärmeerzengung in Niedersachsen (KRALEMANN 2007a)

Mit ca. 7.7 Mio. Fm Wald-Scheitholz liegt der Verbrauch in Süddeutschland (Baden-Württemberg und Bayern) deutlich über dem norddeutschen (SchleswigHolstein, Niedersachsen, Bremen und Hamburg) Scheitholzverbrauch von ca. 1,4 Mio. Fm (MANTAU u. SÖRGEL 2006). 
Für Niedersachsen wurde von KRALEMANN (2007b) für das Jahr 2006 ein Bestand von insgesamt ca. 1.084.365 Einzelöfen und Zentralheizungen angegeben. Mit ca. 97 \% haben die Scheitholzeinzelöfen den größten Anteil. Ihr Bestand ist seit 2002 um über $15 \%$ angestiegen (vgl. Tab. 4).

Der Bestand an Pelleteinzelöfen ist seit dem Jahr 2002 um über 1.700 Öfen gestiegen. Die Anzahl der Pelletzentralheizungen hat sich in den letzten vier Jahren mehr als verzehnfacht und lag im Jahr 2006 bei 2.672 Zentralheizungen (vgl. Tab. 4). Für Deutschland konnten von MANTAU u. SÖRGEL (2006) mit ca. 0,6 Mio. Fm im Jahr 2005 eine Zunahme des Verbrauchsvolumens an Pellets um fast $50 \%$ seit 2000 ermittelt werden.

Tabelle 4: $\quad$ Bestand an Öfen und Zentralheizungen in Niedersachsen im Jahr 2002 und 2006 (nach KRALEMANN 2007b)

\begin{tabular}{l|c|c}
\hline Öfen/Zentralheizungen & Bestand 2002 & Bestand 2006 \\
\hline Pelleteinzelöfen & 285 & 1.987 \\
Hackschnitzelzentralheizungen & 302 & 1.056 \\
Pelletzentralheizungen & 206 & 2.672 \\
Scheitholzeinzelöfen & 909.971 & 1.050 .012 \\
Scheitholzzentralheizungen & 21.769 & 28.638 \\
\hline
\end{tabular}

In Deutschland sind gegenwärtig etwa 95 Biomasse-Heizkraftwerke mit einer elektrischen Leistung von 480 MW in Betrieb (SCHÄFER 2004). In Niedersachsen gibt es fünf Holzheizkraftwerke mit einer elektrischen Leistung von ca. 95 MW. Bei Berücksichtigung der angrenzenden Regionen erhöht sich die Leistung auf ca. 167 MW.

Als Brennstoff (ca. 4,7 Mio.t/a) kommt in Deutschland derzeit fast ausschließlich Altholz zum Einsatz (SCHÄFER 2004). Der jährliche Brennholzbedarf der niedersächsischen Holzheizkraftwerke liegt bei ca. $680.000 \mathrm{t}$ bzw. unter Berücksichtigung der angrenzenden Regionen einiger benachbarter Bundesländer bei ca. 1,3 Mio. t. 


\section{Analyse des Nutzungsverhaltens zwischen BWI 1 und BWI 2}

\subsection{Allgemeines}

Die Ergebnisse der BWI 2 zur Vorratsausstattung der deutschen Wälder haben zunächst vielfach zu einer großen Euphorie geführt, weil sie hohe Nutzungspotenziale an Rohholz ausweisen. Getrübt wurden die daran geknüpften Erwartungen durch die eingeschränkte Verfügbarkeit dieser vorhandenen Rohholzpotenziale. Die Nutzung der Holzvorräte hängt nämlich nicht nur von deren Höhe, sondern maßgeblich von den Nutzungsmöglichkeiten und dem Nutzungswillen der Waldbesitzer ab. Auf Waldflächen, die aus verschiedenen Gründen vorübergehend nicht bestockt (Blößen), nicht begehbar, unproduktiv (dGZ < $1 \mathrm{~m}^{3} / \mathrm{ha}$ ) oder rechtlich nicht nutzbar sind, wird keine Nutzung unterstellt. Selbst die Nutzung der produktiven und begehbaren Waldflächen ist durch vielfältige Faktoren eingeschränkt, die es zu identifizieren gilt. Die potenziell zu nutzende Rohholzmenge ist daher immer nur in Teilen tatsächlich mobilisierbar.

Im Staats- und Körperschaftswald wurde das verfügbare Rohholz-Potenzial in der Vergangenheit vollkommen ausgenutzt (BMELV 2005; POLLEY u. KROIHER 2006). Das ungenutzte Potenzial steht damit im Privatwald. Die Mobilisierung dieser Holzeinschlagsreserven ist gegenwärtig eine große Herausforderung für den Forst- und Holz-Sektor. Deshalb ist es von Bedeutung das Nutzungsverhalten von Waldbesitzern zu analysieren, um Ansatzpunkte für Veränderungen aufzudecken.

Anhand einer statistischen Analyse der BWI-Daten wurden Einflussfaktoren identifiziert, die in der Vergangenheit dazu geführt haben, dass das potenziell nutzbare Holz nicht eingeschlagen wurde. Dadurch werden Ansatzpunkte deutlich, die in Zukunft in Angriff genommen werden müssen, um mehr Flächen und Vorräte der Nutzung zuzuführen.

\subsection{Datengrundlage}

Als Datengrundlage für die Analyse des Nutzungsverhaltens und der Nutzungsintensitäten im Zeitraum 1987 - 2002 dienten alle begehbaren Traktecken Niedersachsens der BWI 1, bestockt mit produktivem Wald ohne Nutzungsverbot. Der zur Auswertung herangezogene Datensatz (3.084 Traktecken) repräsentiert eine Waldfläche von knapp 968.000 ha.

Die Traktecken wurden anhand der größten Standfläche jeweils einem Bestandestyp der Hauptbaumarten zugeordnet. Die im Folgenden vorgestellten Auswertungen beziehen sich daher auf Bestandestypen und nicht auf einzelne 
Anteilsflächen der Baumartengruppen. Dabei wurden fünf Bestandestypen unterschieden:

- Bestände mit vorherrschender Buche (Buche und Laubholz mit hohem Umtrieb)

- Bestände mit vorherrschender Eiche

- Bestände mit vorherrschendem Laubholz mit niedrigem Umtrieb

- Bestände mit vorherrschender Fichte (Fichte, Douglasie und Tanne)

- Bestände mit vorherrschender Kiefer (Kiefer und Lärche)

3.3 Vergleich der durch BWI 1 und BWI 2 dokumentierten Waldzustände und Nutzungsintensitäten

Anhand der BWI-Daten wurde für jede Traktecke der Vorrat pro Hektar zum Zeitpunkt 1987 und 2002 sowie der Einschlag pro Hektar im Zeitraum 1987 bis 2002 berechnet. Die Anfangs-, End- und Veränderungszustände ließen eine nach Altersklassen geordnete Darstellung getrennt nach Bestandestyp und Eigentumsart zu, wodurch eine differenzierte Analyse der Nutzungsintensitäten möglich war. Beim Vergleich der Eigentumsarten wurden die Ergebnisse des Staatswaldes (Landes- und Bundeswald) den zusammengefassten Ergebnissen des Privat- und Körperschaftswaldes gegenübergestellt.

Der Repräsentationsfaktor einer Traktecke, der die unterschiedliche Stichprobendichte der BWI im Flach- und Bergland berücksichtigt, ermöglichte es, die repräsentierte Fläche pro Altersklasse zum Zeitpunkt 1987 und 2002 hochzurechnen. Durch Einschlag, Zuwachs und natürlichen Abgang im Betrachtungszeitraum fanden innerhalb der Bestandestypen Verschiebungen der Flächenausstattungen statt, die zu einem Flächenzugang oder -abgang bei den Bestandestypen führten. Tabelle 5 zeigt die absolute Veränderung der Flächenausstattung von Bestandestypen zwischen 1987 und 2002 über alle Eigentumsarten.

Tabelle 5: $\quad$ Absolute Veränderung der Flächenausstattung von Bestandestypen zwischen 1987 und 2001

\begin{tabular}{l|c|c|c|c|c}
\hline & Buche & Eiche & Aln & Fichte & Kiefer \\
\hline Fläche BWI 1 (ha) & 153.099 & 107.754 & 126.795 & 193.444 & 386.797 \\
Fläche BWI 2 (ha) & 157.289 & 127.111 & 105.075 & 184.229 & 388.937 \\
\hline $\begin{array}{l}\text { Differenz } \\
\text { (BWI 2-BWI 1) }\end{array}$ & $\mathbf{4 . 1 9 0}$ & $\mathbf{1 9 . 3 5 7}$ & $\mathbf{- 2 1 . 7 2 0}$ & $\mathbf{- 9 . 2 1 6}$ & $\mathbf{2 . 1 4 0}$ \\
\hline
\end{tabular}


Bestände mit vorherrschender Fichte oder Laubholz niedrigen Umtriebs waren 2002 mit weniger Fläche vertreten als noch 1987. Dieser Flächenverlust verlief zugunsten von Buchen-, Kiefern- und insbesondere Eichenbeständen.

\subsubsection{Vorrats-, Alters- und Grundflächenveränderung zwischen 1987 und 2002}

Der Vorrat, berechnet über alle Traktecken, hat bei allen Bestandestypen von 1987 bis 2002 zugenommen (vgl. Tab. 6). Im Vergleich der Eigentumsarten weist der Privat- und Körperschaftswald über alle Bestandestypen hinweg einen höheren Vorrat je Hektar auf. Dabei ist auch die Vorratszunahme im Privatwald höher als im Staatswald. Die höchste Vorratszunahme zeigt sich in Beständen mit vorherrschendem Laubholz niedrigen Umtriebs.

Tabelle 6: $\quad$ Vorrat von Bestandestypen zum Zeitpunkt 1987 und 2002 aller Traktecken

\begin{tabular}{l|c|c|c|c}
\hline \multirow{2}{*}{ Bestandestyp } & \multicolumn{3}{|c}{ Vorrat (Vfm/ha) alle Traktecken } \\
& \multicolumn{2}{|c}{ Staatswald } & \multicolumn{2}{c}{$\begin{array}{c}\text { Privat- und } \\
\text { Körperschaftswald }\end{array}$} \\
\hline \multirow{2}{*}{ Buche } & 1987 & 2002 & 1987 & 2002 \\
Eiche & 255 & 315 & 315 & 370 \\
Aln & 245 & 285 & 285 & 370 \\
Fichte & 90 & 195 & 135 & 250 \\
Kiefer & 230 & 305 & 160 & 270 \\
\hline
\end{tabular}

Ein Vergleich des flächengewogenen Durchschnittsalters der Bestandestypen 1987 und 2002 zeigt, dass sich der Vorratsaufbau im Betrachtungszeitraum vorrangig durch eine Erhöhung des Durchschnittsalters erklären lässt (vgl. Tab. 7). Dabei unterscheiden sich die Durchschnittsalter der Bestandestypen der jeweiligen Eigentumsarten nur wenig. Lediglich die Fichte im Privat- und Körperschaftswald weist im Vergleich zum Staatswald ein deutlich geringeres Durchschnittsalter auf. 
Tabelle 7: $\quad$ Flächengewogenes Durchschnittsalter der Bestandestypen 1987 und 2002 getrennt nach Eigentumsarten

\begin{tabular}{l|c|c|c|c}
\hline & \multicolumn{3}{|c}{ Flächengewogenes Durchschnittsalter (Jahre) } \\
\hline Bestandestyp & \multicolumn{2}{|c|}{ Staatswald } & Privat- und Körperschaftswald \\
\hline \multirow{2}{*}{ Buche } & $\mathbf{1 9 8 7}$ & $\mathbf{2 0 0 2}$ & $\mathbf{1 9 8 7}$ & $\mathbf{2 0 0 2}$ \\
Eiche & 82 & 88 & 86 & 93 \\
Aln & 80 & 87 & 76 & 88 \\
Fichte & 33 & 47 & 30 & 42 \\
Kiefer & 50 & 62 & 37 & 45 \\
\hline
\end{tabular}

Die Höhe der Nutzungsintensität kann über die Veränderung der Grundfläche der Traktecken, auf denen Probebäume im Untersuchungszeitraum ausgeschieden sind, bestimmt werden (vgl. Abb. 19). Für jede dieser Traktecken wurde im Anhalt an POLLEY und KROIHER (2006) ein Grundflächenindex berechnet, der als Quotient aus der Grundfläche je Hektar der BWI 2 und der BWI 1 die Nutzungsintensität der zurückliegenden Periode widerspiegelt.

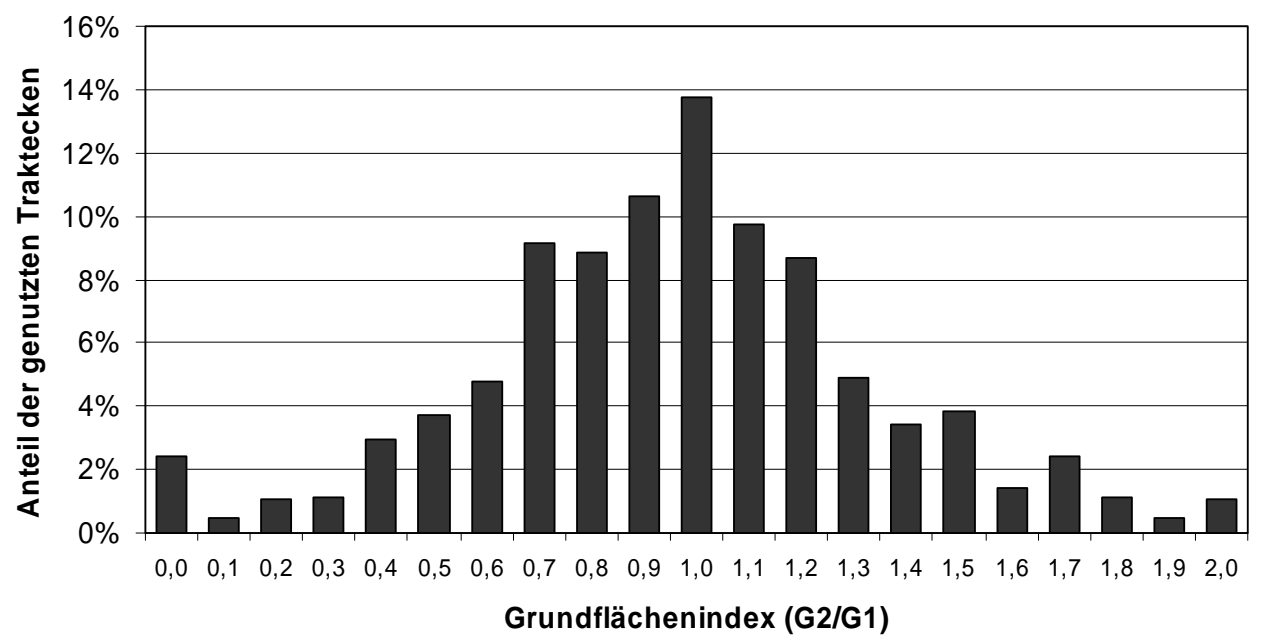

Abbildung 19: Anteil der genutz̨ten Traktecken in Abhängigkeit vom Grundflächenindex (G2/G1) 
Auf Traktecken mit einem Grundflächenindex von 0,0 sind vom Zeitpunkt der BWI 1 zum Zeitpunkt der BWI 2 sämtliche Probebäume ausgeschieden und bis zur BWI 2 hatten die neuen Bäume auf der Fläche entweder nicht die Aufnahmeschwelle überschritten oder aber die Fläche war bis dato noch unbestockt. Hingegen ist ein Grundflächenindex von 2,0 als eine Verdopplung der TrakteckenGrundfläche zu verstehen. Ein Grundflächenindex von 1,0 wiederum bedeutet, dass sich die durch Zuwachs bedingte Grundflächenzunahme mit der durch das Ausscheiden von Bäumen einhergehenden Abnahme zwischen 1987 und 2002 die Waage halten. In Abbildung 19 wird deutlich, dass die Verteilung über den Grundflächenindices leicht linksschief ist (mittlerer Grundflächenindex $=0,93$ ) und es damit auf $45 \%$ der Traktecken zu einer Absenkung der Grundfläche kam. Auf $14 \%$ blieb das Verhältnis gleich, auf $41 \%$ stieg die Grundfläche an.

Die Holzernte wurde auf den niedersächsischen Probepunkten im Zeitraum 1987 bis 2002 vorwiegend als einzelstamm- bis gruppenweiser Eingriff durchgeführt. Nichtsdestotrotz fanden auch flächige Endnutzungen statt, die planmäßig, aber auch in Folge von Schadereignissen, stattgefunden haben. In Anhalt an POLLEY und KROIHER (2006) sind die Traktecken mit einem Grundflächenindex bis 0,25 als flächige Endnutzung interpretiert worden. Da eine Traktecke nur eine kleine Fläche des sie umgebenen Gesamtbestandes repräsentiert, kann die flächige Nutzung eine Auflichtung innerhalb des Bestandes sein und muss nicht zwingend einen Kahlschlag des Gesamtbestandes bedeuten. In Niedersachsen sind nach der Definition von POLLEY knapp $4 \%$ der genutzten Fläche flächig endgenutzt worden. $30 \%$ dieser Endnutzungsflächen liegen im Staatswald, 50\% im Privatund $20 \%$ im Körperschaftswald. $80 \%$ betrafen Nadelholz-, $20 \%$ Laubholzbestände.

\subsubsection{Vergleich der Altersklassenstruktur des Vorrates in Beständen mit vorherr- schender Buche}

Im Staatswald zeigen sich in Beständen mit führender Buche zwei Eingriffsschwerpunkte im Alter 61-80 und 101-120, die mit einem Vorratsabbau zwischen 1987 und 2002 einhergehen (vgl. Abb. 20). Dies ist entsprechend den Zielvorgaben der Niedersächsischen Landesforsten zum einen auf die erhöhte Durchforstungsstärke in der mittleren Altersklasse bzw. auf die Zielstärkennutzung in der höheren Altersklasse zurückzuführen. In den übrigen Altersklassen hingegen fand ein deutlicher Vorratsaufbau statt. Der hohe Vorrat in der höchsten Altersklasse ist z. T. bedingt durch das Belassen von starkem, teilweise rotkernigem Altholz auf der Fläche im Zuge von Naturschutzmaßnahmen. Über alle Bestandestypen, insbesondere mit führendem Nadelholz, zeigt sich 2002 ein Rückgang der Waldfläche in der ersten Altersklasse. Dies erklärt sich durch die naturnahe, kahlschlagfreie Bewirtschaftung in der Beobachtungsperiode und die damit einhergehenden längeren Verjüngungszeiträume. 
Die jährliche Einschlagsmenge aus den Buchenbeständen lag zwischen BWI 1 und BWI $2 \mathrm{im}$ Staatswald im Mittel bei 5,3 Efm/ha. Zu einer nennenswerten Flächenverschiebung zwischen den Altersklassen kam es nicht.

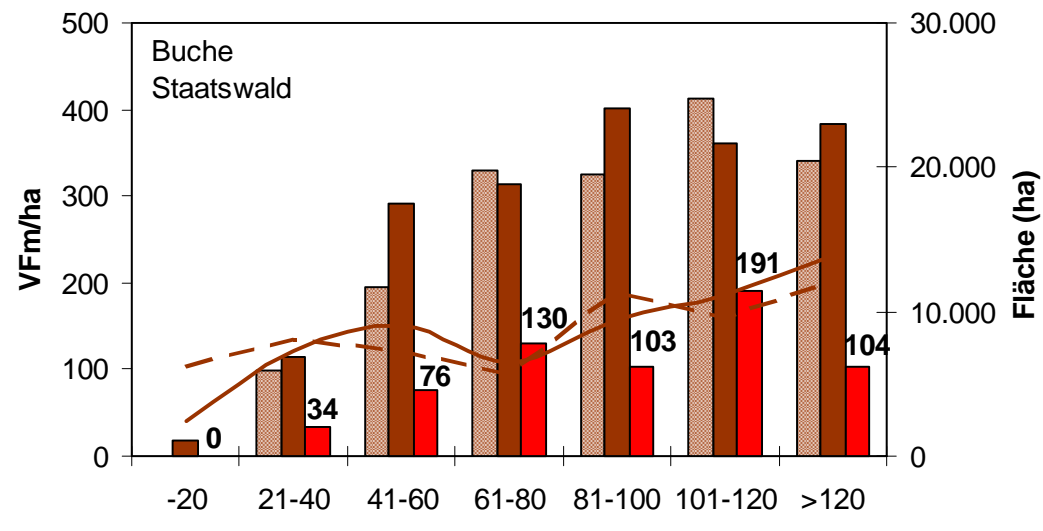

Altersklasse (Jahre)

Vorrat 1987

Vorrat 2002

Nutzung 1987-2002

— - Fläche 1987

Fläche 2002

Abbildung 20: Vorrat und Nutzung (in V fm/ ha) sowie Flächenausstattung in Beständen mit vorherrschender Buche nach Altersklassen im Staatswald

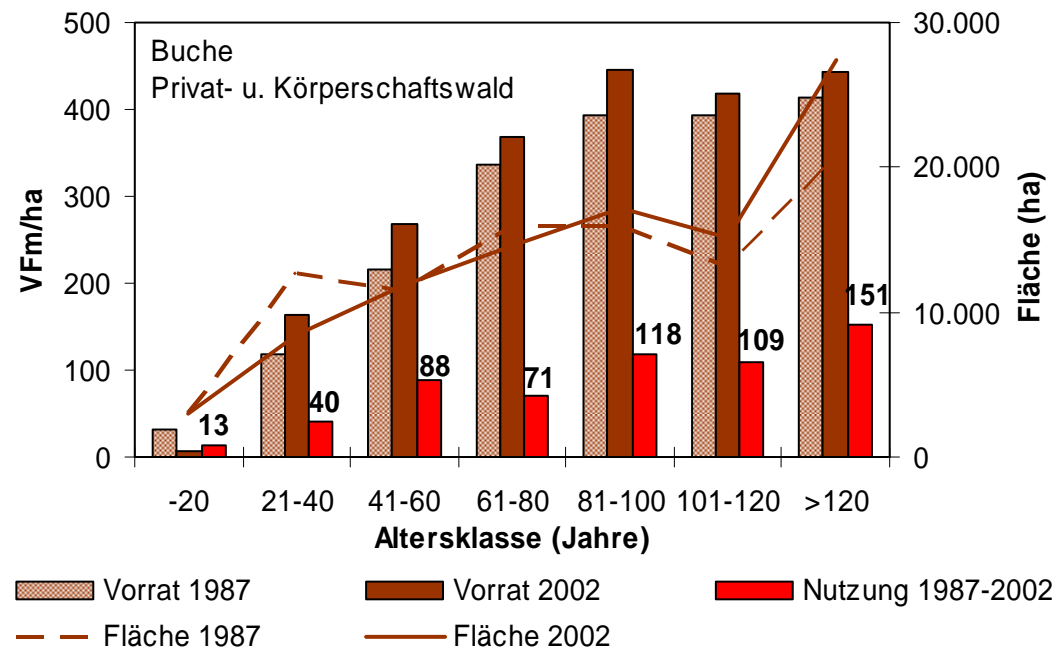

Abbildung 21: Vorrat und Nutzung (in V fm/ha) sowie Flächenausstattung in Beständen mit vorherrschender Buche nach Altersklassen im Privat-u. Körperschaftswald 
Im Privat- und Körperschaftswald war der Vorrat in Beständen mit vorherrschender Buche 2002 in allen Altersklassen deutlich höher als 1987 (vgl. Abb. 21). Zudem bestehen im Vergleich zum Staatswald wesentlich höhere Vorratsausstattungen in den Beständen älter als 80 Jahre. Mit 5,5 Efm/ha lag der mittlere Einschlag im Betrachtungszeitraum geringfügig über dem des Staatswaldes. Eine erwähnenswerte Flächenverschiebung zwischen den Altersklassen fand ebenfalls nicht statt.

\subsubsection{Vergleich der Altersklassen- und Durchmesserklassenstruktur des Vorrates in Beständen mit vorherrschender Eiche}

In den Beständen mit vorherrschender Eiche ist im Staatswald der Vorrat zwischen BWI 1 und BWI 2 mit Ausnahme der Altersklasse IV (61-80 J.) durchweg angestiegen (vgl. Abb. 22). Der Vorratsabbau in der vierten Altersklasse erklärt sich durch die verstärkte Nutzungsintensität in den Beständen mit vorherrschender Eiche bei Durchforstungseingriffen in dieser Altersphase. Im Staatswald lag der jährliche Einschlag im Mittel bei 3,7 Efm/ha.

Im Privat- und Körperschaftswald ist in Beständen mit vorherrschender Eiche die Vorratsausstattung sowohl zum Zeitpunkt 1987 als auch 2002 ab dem Alter 21 über alle Altersklassen deutlich höher als im Staatswald (vgl. Abb. 23).

Bis zum Alter 120 hat z. T. ein deutlicher Vorratsaufbau stattgefunden. Nur in Beständen älter als 120 Jahre kam es auf Grund höherer Nutzungsintensität zu einem Vorratsabbau. Bei diesen Beständen handelt es sich fast ausschließlich um Eichenbestände mit Buchenmischung. Es ist davon auszugehen, dass hier im Zuge der Altdurchforstung verstärkt Eichen freigestellt wurden. Die jährliche Nutzungsintensität lag in Beständen mit führender Eiche im Privat- und Körperschaftswald im Mittel bei 2,8 Efm/ha, war also um fast einen Festmeter geringer als im Staatswald. 

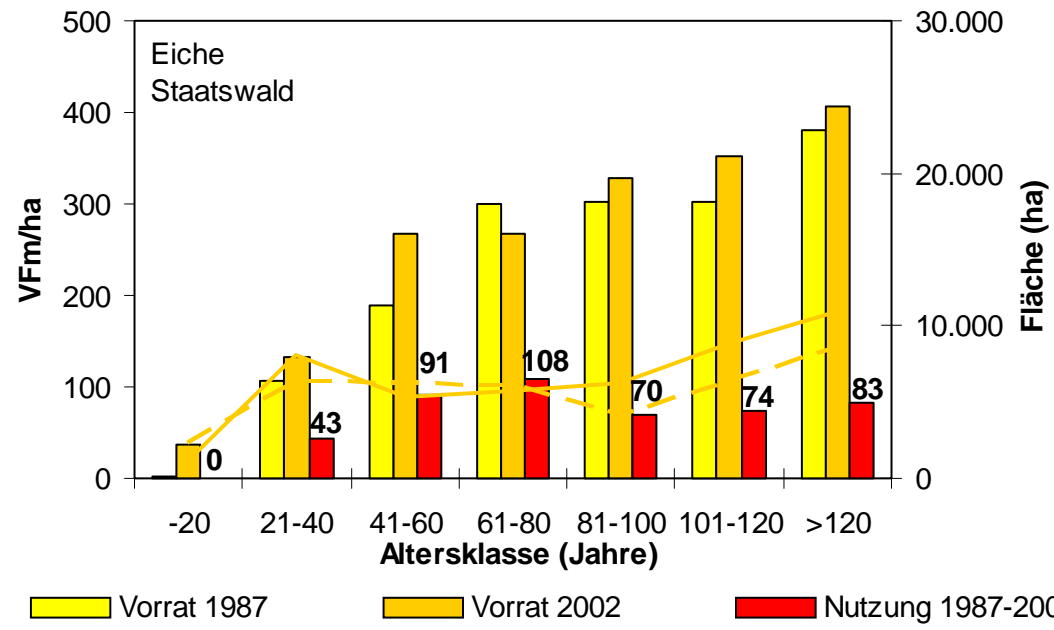

Vorrat 2002

Nutzung 1987-2002

— - Fläche 1987

Fläche 2002

Abbildung 22: Vorrat und Nutzung (in V fm/ ha) sowie Flächenausstattung in Beständen mit vorberrschender Eiche nach Altersklassen im Staatswald

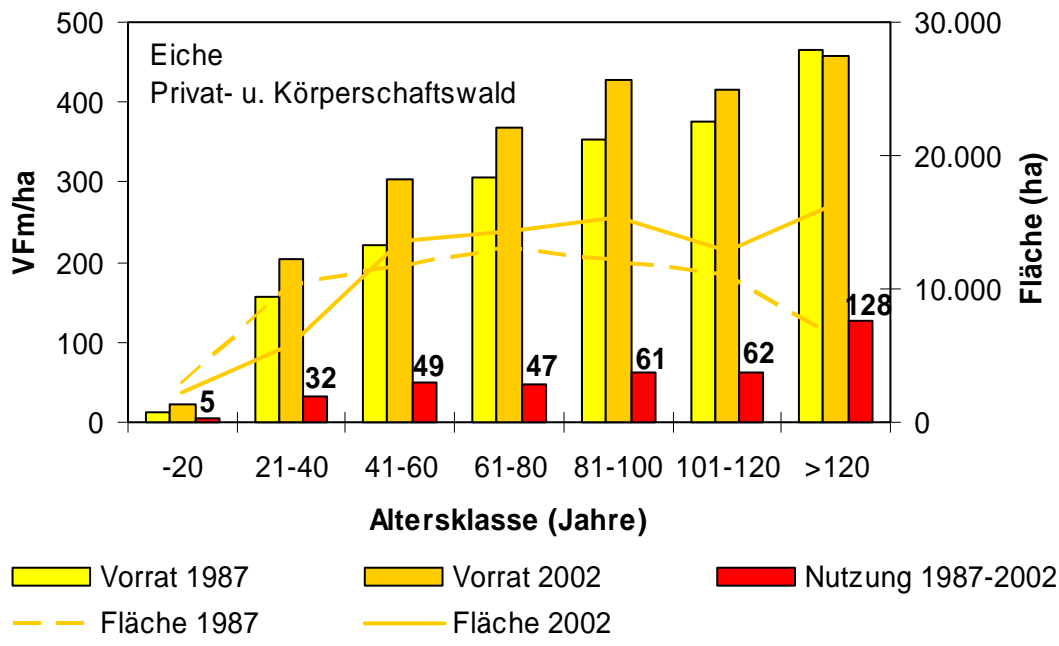

Abbildung 23: Vorrat und Nutzung (in Vfm/ha) sowie Flächenausstattung in Beständen mit vorherrschender Eiche nach Altersklassen im Privat- und Körperschaftswald 


\subsubsection{Vergleich der Altersklassen- und Durchmesserklassenstruktur des Vorrates in Beständen mit vorberrschendem Laubholz niedrigen Umtriebs}

In den Beständen mit führendem Laubholz niedrigen Umtriebs hat bei einem sehr geringen jährlichem mittleren Einschlag von $1 \mathrm{Efm} / \mathrm{ha}$ im Staatswald und $1,2 \mathrm{Efm} / \mathrm{ha}$ im Privat- und Körperschaftswald bei allen Eigentumsarten und über alle Altersklassen hinweg ein Vorratsaufbau stattgefunden (vgl. Abb. 24). Der Vorratsaufbau ist in Beständen älter als 60 Jahre am stärksten und führte im Privatund Körperschaftswald zu einer annähernden Verdoppelung des Vorrates. Die Flächenanteile dieser Altersklasse sind jedoch auf Grund der kurzen Umtriebszeit gering.
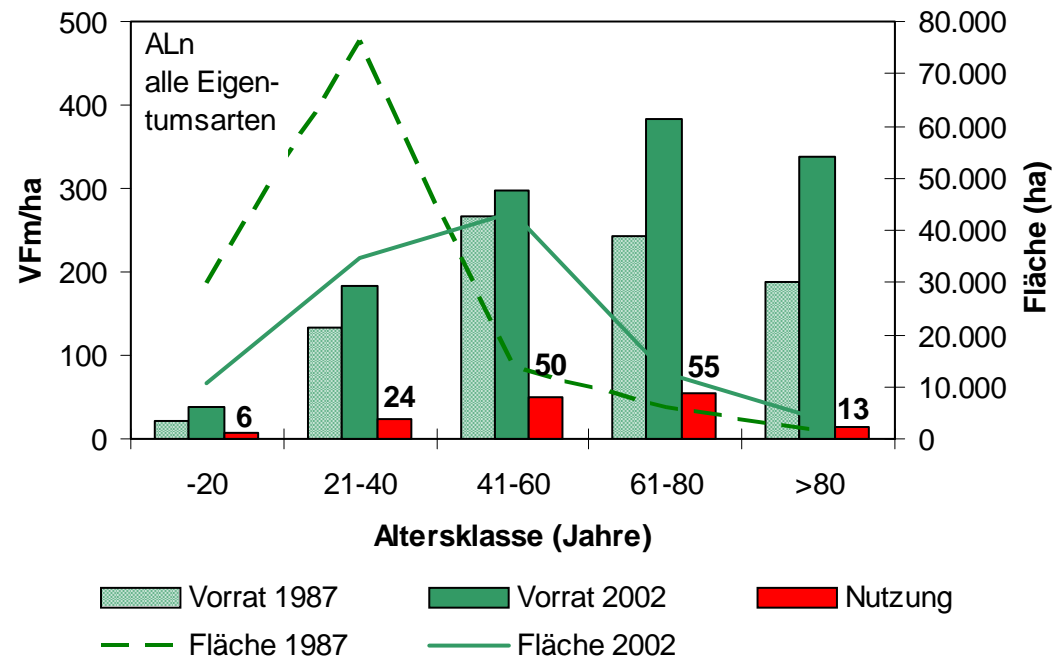

Abbildung 24: Vorrat und Nutzung (in Vfm/ha) sowie Flächenausstattung in Beständen mit vorherrschendem Laubholz niedriger Lebensdauer nach Altersklassen in allen Eigentumsarten

\subsubsection{Vergleich der Altersklassen- und Durchmesserklassenstruktur des Vorrates in Beständen mit vorherrschender Fichte}

In Beständen mit vorherrschender Fichte des Staatswaldes kam es zu einer deutlichen Flächenverschiebung zugunsten älterer Bestände ab Altersklasse III (41$60 \mathrm{~J}$.$) .$

Im Staatswald sind Bestände mit vorherrschender Fichte bis zum Alter 60 zum Zeitpunkt 2002 vorratsreicher als 1987. In der Altersklasse 61-80 hat sich die Vorratsausstattung nicht verändert, in Beständen älter 80 Jahre hingegen nahm der Vorrat auf Grund höherer Nutzungsintensitäten deutlich ab. Die Vorratsabnahme in diesen Beständen kann als Folge des Zielstärkennutzungskonzepts der Nieder- 
sächsischen Landesforsten gedeutet werden. Der jährliche Einschlag lag im Staatswald im Mittel bei 6,1 Efm/ha (vgl. Abb. 25).
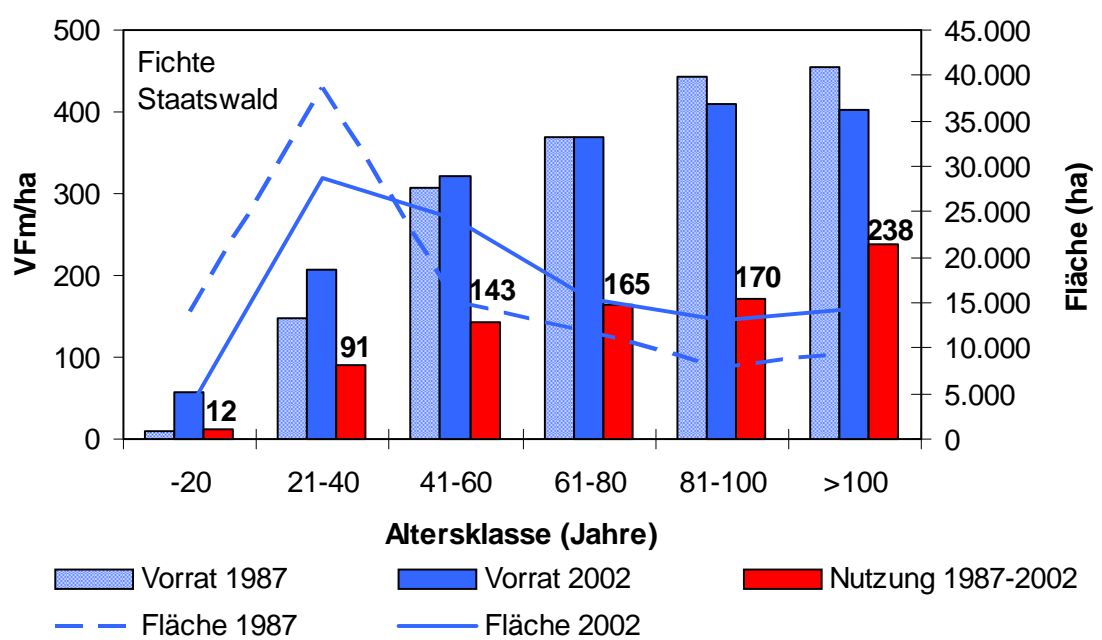

Abbildung 25: Vorrat und Nutzung (in Vfm/ha) sowie Flächenausstattung in Beständen mit vorherrschender Fichte nach Altersklassen im Staatswald

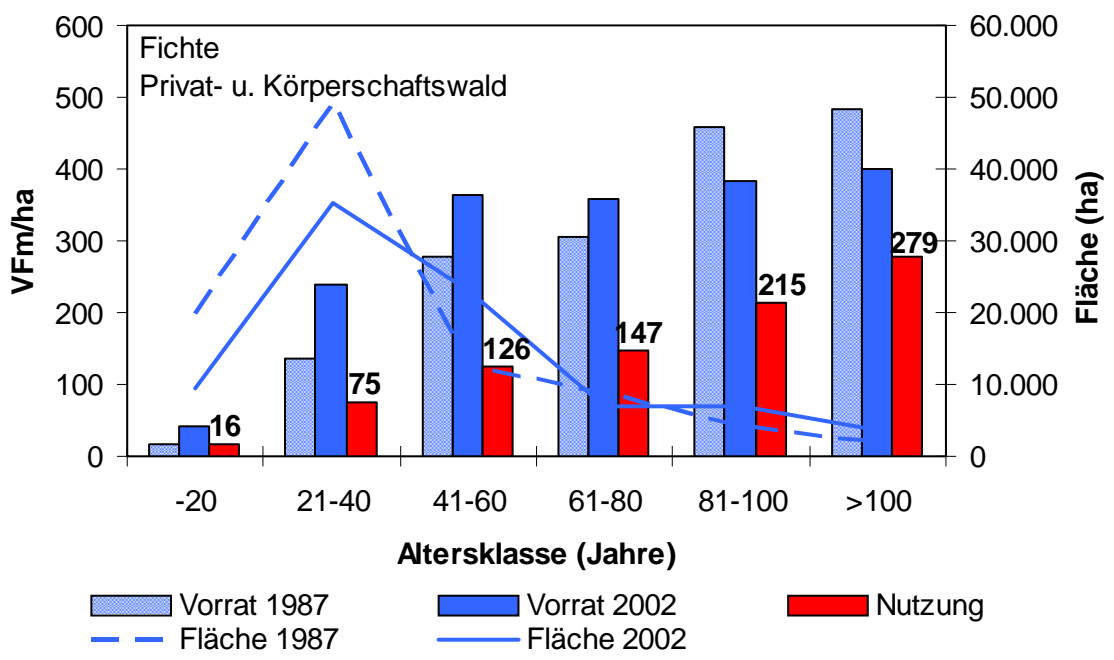

Abbildung 26: Vorrat und Nutzung (in Vfm/ ha) sowie Flächenausstattung in Beständen mit vorberrschender Fichte nach Altersklassen im Privat- und Körperschaftswald 
Im Privat- und Körperschaftswald ist der Vorratsaufbau in den jungen und mittelalten Beständen bis zum Alter 80 wesentlich stärker ausgeprägt als im Staatswald (vgl. Abb. 26). Somit liegen hier echte Durchforstungsreserven vor, die sich wiederum ausschließlich in den bisher gar nicht oder mit geringer Intensität genutzten Flächen des Privat- und Körperschaftswaldes befinden. Ab dem Alter 80 wurde auch im Privat- und Körperschaftswald der Hektarvorrat abgebaut. Dabei war die Nutzungsintensität sogar höher als im Staatswald. Im Mittel aller Altersklassen wurden im Privat- und Körperschaftswald jährlich 4,3 Efm/ha eingeschlagen, fast $2 \mathrm{Efm} / \mathrm{ha}$ weniger als im Staatswald.

\subsubsection{Vergleich der Altersklassen- und Durchmesserklassenstruktur des Vorrates in Beständen mit vorherrschender Kiefer}

Beim Bestandestyp mit vorherrschender Kiefer liegen fast $3 / 4$ der Flächen im Privat- und Körperschaftswald und nur etwas über 1/4 der Flächen im Staatswald. Bei beiden Eigentumsgruppen kam es zu einer Flächenverschiebung zugunsten der Bestände $a b$ der Altersklasse 41-60. Die Ursache liegt in den Nachkriegsaufforstungen und den Erstaufforstungen von Heide- und Moorflächen.

In Beständen des Staatswaldes hat sich bis zum Alter 80 zwischen 1987 und 2002 ein Vorratsaufbau vollzogen (vgl. Abb. 27). In älteren Beständen gab es bei einer deutlich höheren Nutzungsintensität keine merkliche Veränderung in der Vorratsausstattung. Im Mittel lag der jährliche Einschlag bei 4,0 Efm/ha im Staatswald.

Im Privat- und Körperschaftswald ist der Vorratsaufbau im Vergleich zum Staatswald stärker ausgeprägt und erstreckt sich über alle Altersklassen. Echte Nutzungsreserven finden sich demnach auch hier lediglich im bisher wenig und ungenutzten Privat- und Körperschaftswald.

Die Nutzungsintensität in Beständen mit vorherrschender Kiefer ist mit einem jährlichen Einschlag von im Mittel 2,8 Efm/ha geringer als im Staatswald, jedoch sind deutlich höhere Einschlagsmassen pro Hektar als im Staatswald in Beständen älter als 100 Jahre zu verzeichnen (vgl. Abb. 28). 


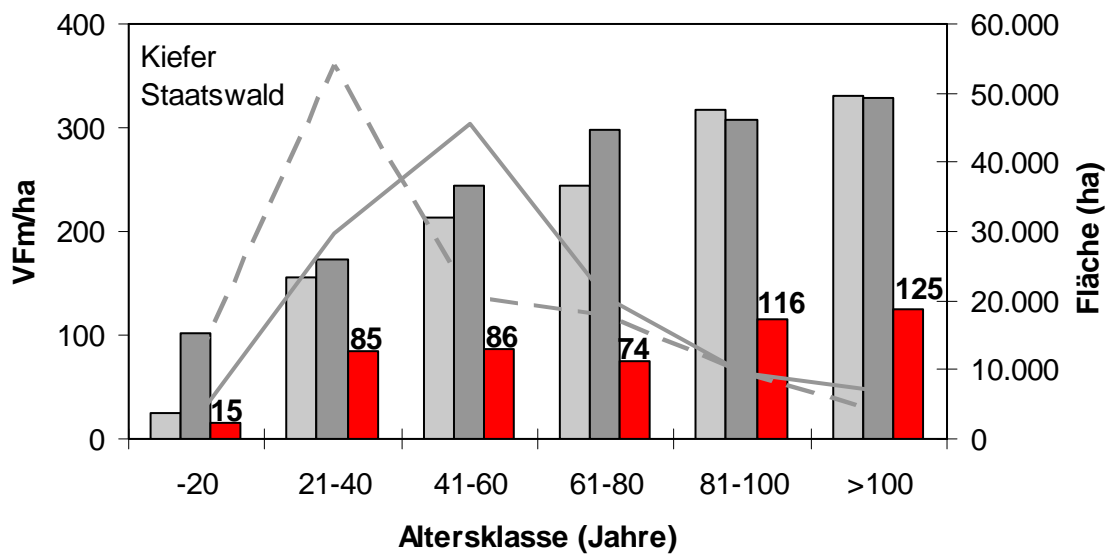

$\square$ Vorrat 1987
$-\quad$ Fläche 1987

Vorrat 2002

Nutzung 1987-2002

Fläche 2002

Abbildung 27: Vorrat und Nutzung (in Vfm/ha) sowie Flächenausstattung in Beständen mit vorherrschender Kiefer nach Altersklassen im Staatswald
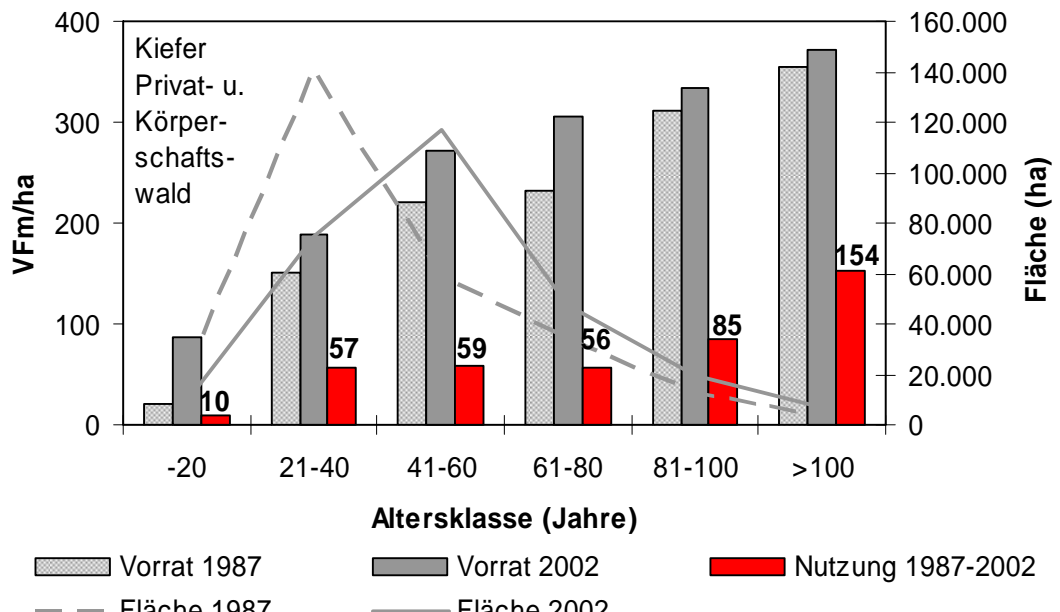

Nutzung 1987-2002

_ - Fläche 1987

Fläche 2002

Abbildung 28: Vorrat und Nutzung (in Vfm/ha) sowie Flächenausstattung in Beständen mit vorherrschender Kiefer nach Altersklassen im Privat- und Körperschaftswald 


\subsection{Einflussfaktoren auf das Nutzungsverhalten zwischen 1987 und 2002}

Die ausschlaggebenden Faktoren für eine geringe oder unterlassene Nutzung von Waldflächen innerhalb des 15-jährigen Zeitraums zwischen BWI 1 und BWI 2 sind mannigfaltig. Die Identifikation dieser Einflussfaktoren bietet Ansatzpunkte für gezielte Mobilisierungsmaßnahmen durch die Politik bzw. die den Waldbesitz jeweils betreuenden Dienststellen. Die das Nutzungsverhalten beeinflussenden Faktoren lassen sich verschiedenen Kategorien zuordnen (vgl. Abb. 29):

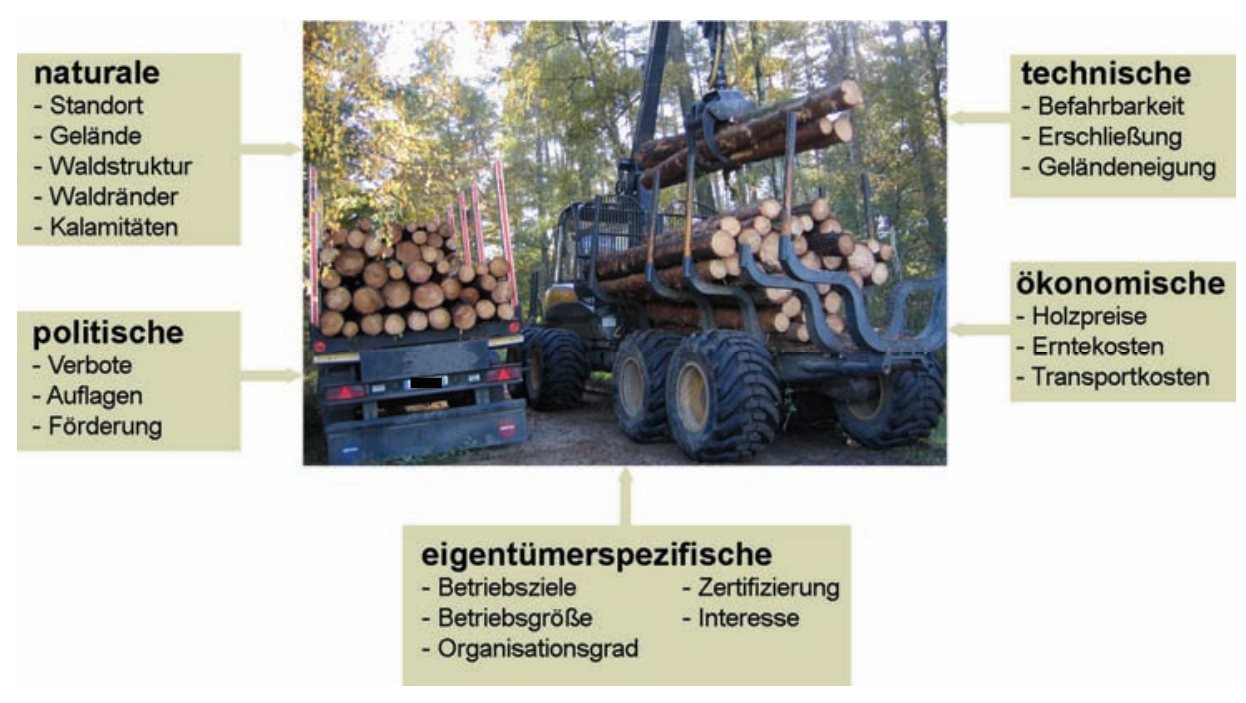

Abbildung 29: Einflussfaktoren auf das Nutzungsverhalten von Waldbesitzern (Foto: C. Kleinschmit)

Die Stärke der naturalen, politischen, eigentümerspezifischen, technischen und ökonomischen Einflüsse ist abhängig von der jeweiligen Bestandessituation sowie den ökonomischen und sozioökonomischen Rahmenbedingungen. Dabei beeinflussen sich die Faktoren oft gegenseitig, wodurch die Selektion signifikanter Einflussfaktoren schwierig ist. So erlauben z. B. die Nährstoff- und Wasserversorgung sowie Lage eines Standortes Rückschlüsse auf das Leistungsvermögen des aufstockenden Bestandes wie auch auf die Einsatzmöglichkeiten technischer Systeme.

Von einem Nutzungsverbot produktiver Waldflächen sind in Niedersachsen hauptsächlich Landeswaldflächen betroffen, dazu gehören Teile des Nationalparks Harz und die ausgewiesenen Naturwälder. Die unter Nutzungsverbot stehenden produktiven Waldflächen des Privat- und Körperschaftswaldes sind verschwindend gering. 
Niedersachsen hat einen Vorrat von 8,9 Mio. Vfm starkem Totholz, welches einer Nutzung entgeht (NDS. MiNISTERIUM F. D. LÄNDL. RAUM etc. 2004). Inklusive schwachem Totholz sind es nach Schätzungen des PEFC (REGIONALE PEFCARBEITSGRUPPE NIEDERSACHSEN 2005) 5-15 \% des Gesamtvorrates. Beim Laubholz gibt es sehr viel Starkholz in den Wäldern, das bisher häufig ungenutzt blieb, weil keine Nachfrage bestand. Je weiter sich die Nutzung verzögert, desto schwieriger wird es, die Laubstarkholzvorräte wirtschaftlich und technisch zu verwerten. Hinzu kommt die Einschränkung, dass Althölzer, die als Horst- und Brutbäume dienen, einen rechtlichen Schutz genießen (NNatG, \37, Abs. 4).

Niedersachsen hat, insbesondere durch die kleinparzellierten Wälder im westlichen Tiefland, 88.000 ha Waldrand, was insgesamt $8 \%$ der Waldfläche entspricht (NDS. MinisteriUM F. D. LÄNDL. RAUM etc. 2004). Mit 114 m Waldrand je Hektar ist der Privatwald besonders durch Holzproduktionsverluste betroffen.

\subsection{Statistische Analyse des Nutzungsverhaltens}

Die statistische Analyse des Nutzungsverhaltens zur Ermittlung signifikanter Einflussfaktoren erfolgte mittels logistischer Regressionen ähnlich der Methode, wie sie von BORCHERT bei der Bayerischen Holzaufkommensprognose angewandt wurde (BAYER. LANDESANSTALT F. WALD U. FORSTWIRTSCHAFT 2005). Da im Rahmen der BWI lediglich die Nutzung von Bäumen ab $10 \mathrm{~cm}$ BHD erfasst wurde, sind in die Analyse nur Traktecken der BWI 1 einbezogen worden, die mit Bäumen oberhalb der Derbholzgrenze bestockt waren. Der zur statistischen Analyse herangezogene Datensatz enthält 2.797 Traktecken und repräsentiert eine Waldfläche von 883.000 ha.

Anhand der Nutzung von Bäumen im Zeitraum 1987 bis 2002 wurden die Traktecken in jene mit oder ohne Holznutzung unterteilt. Die zu erklärende Variable ist binär: Fehlen 2002 auf einer Traktecke ein oder mehrere Probebäume durch Holznutzung - ja oder nein? Dabei ist zu berücksichtigen, dass das Vorhandensein aller Probebäume auf einer Traktecke zum Zeitpunkt 2002 nicht gleichbedeutend mit einer unterbliebenen Nutzung des umgebenen Bestandes ist. Dies hat verschiedene Gründe: Nutzungen erfolgen nicht immer ganzflächig, sondern z. T. auch nur geklumpt und punktuell. Die durchschnittliche Probebaumstammzahl pro Traktecke beträgt bedingt durch die Winkelzählbreite 4 bei den BWI-Aufnahmen sieben Bäume. Die Wahrscheinlichkeit, dass, obwohl eine Nutzung auf der repräsentierten Fläche stattfand, keiner dieser sieben Bäume entnommen wurde, ist vergleichsweise hoch. Folglich kann nicht unmittelbar aus der Nichtnutzung der Probebäume auf die Fläche ohne Holznutzung hochgerechnet werden. Bedingt durch die Inventurmethode wurden durch die Winkelzählbreite 4 nur Nutzungen ab einer Grundflächenentnahme von $4 \mathrm{~m}^{2} /$ ha auf einer Traktecke erfasst. Auf Traktecken, auf denen zwischen 1987 und 2002 nach BWI-Angaben kein Baum geerntet wurde, kann durchaus eine geringe Nutzung von weniger als 
$4 \mathrm{~m}^{2}$ /ha stattgefunden haben. Es lässt sich dabei nicht unterscheiden, auf welchen Traktecken zwischen 1987 und 2002 keine Nutzungen erfolgten und auf welchen Traktecken durch die Winkelzählbreite 4 die erfolgten Nutzungen nicht erfasst wurden. Werden alle nicht genutzten Traktecken als nicht genutzte Fläche interpretiert, wird die genutzte Fläche systematisch unterschätzt. Gleichwohl liefert der Anteil der genutzten bzw. nicht genutzten Traktecken wichtige Hinweise auf die relative Nutzungsintensität. Dies soll im Folgenden erläutert werden.

In der Abbildung 30 werden die ungenutzten Flächenanteile (Nutzung $<4 \mathrm{~m}^{2} /$ ha) getrennt nach Eigentumsarten dargestellt. Hierbei wird trotz der zuvor angesprochenen Probleme mit der Flächenrepräsentativität deutlich, dass es über alle Baumarten vor allem im Kleinprivatwald zu Nutzungsversäumnissen gekommen ist. Im Mittel erfolgte dort auf $60 \%$ der Flächen keine Nutzung $\left(>4 \mathrm{~m}^{2} / \mathrm{ha}\right.$ ). Im Staatswald hingegen waren je nach Bestandestyp nur 25-35\% der Fläche aus der Nutzung genommen.

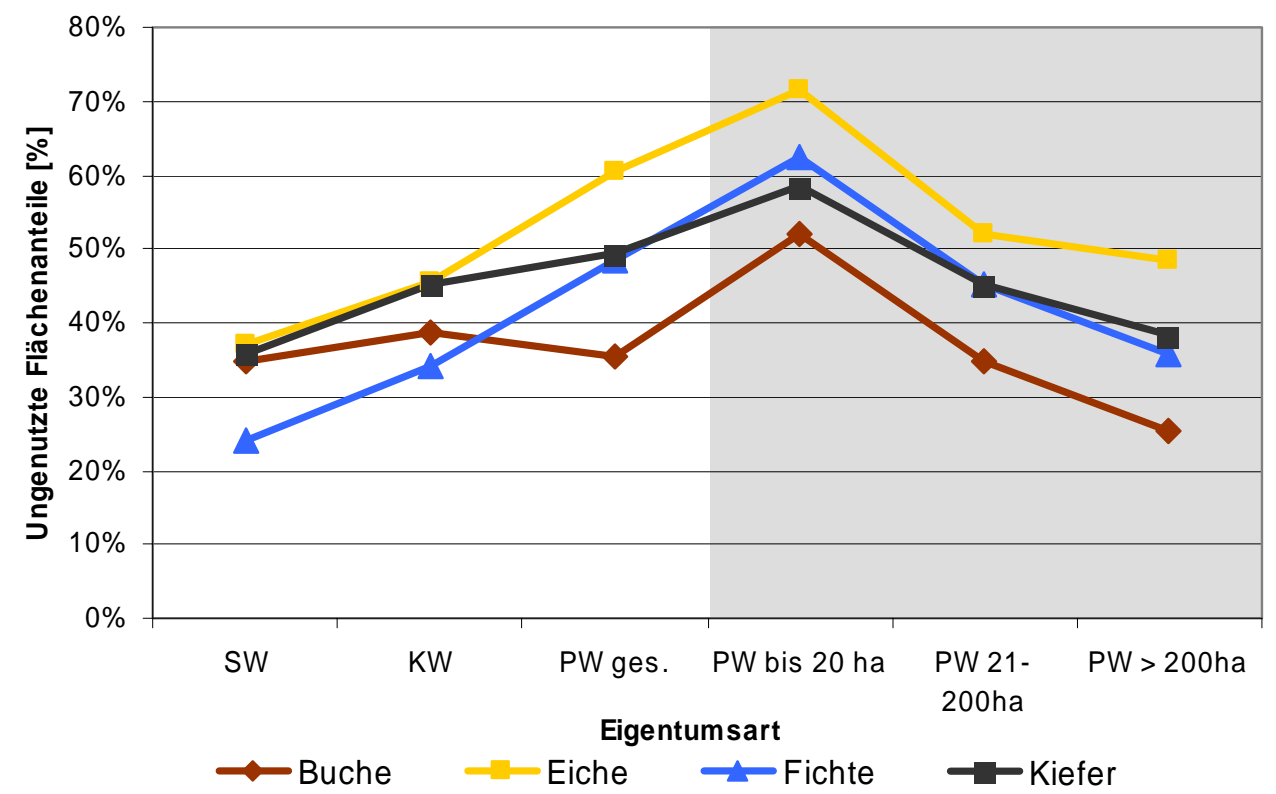

Abbildung 30: Flächenanteile der Bestandestypen getrennt nach Eigentumsarten (SW = Staatswald; $K W=$ Körperschaftswald; $P W=$ Privatwald)

Um die Abhängigkeit des Ausscheidens von Probebäumen von verschiedenen Faktoren (Eigentumsverhältnisse, Lage und Standort des Traktes, Dimensionen des aufstockenden Bestandes) testen zu können, erfolgte eine Auswertung differenziert nach verschiedenen erklärenden Variablen. Abbildung 31 zeigt beispielsweise, dass der Anteil der Flächen mit genutzten Probebäumen in allen Eigentumsarten mit zunehmendem Vorrat pro Hektar steigt. Staats- und Privat- 
wald mit einer Betriebsgröße über 200 ha verhalten sich in ihrer Nutzungsintensität annähernd gleich. Die Nutzungsintensität des Körperschaftswaldes liegt im Mittel $10 \%$ darunter und die des Privatwaldes mit einer Betriebsgröße bis 200 ha ist im Schnitt $20 \%$ geringer.

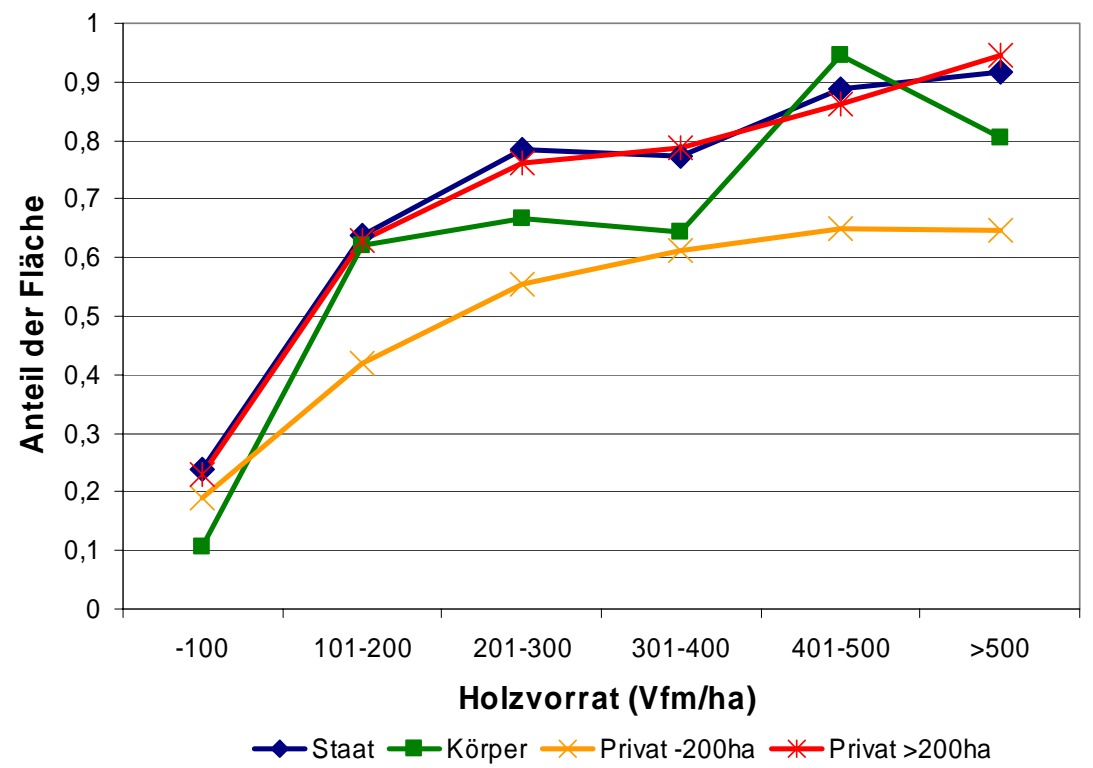

Abbildung 31: Anteil der Fläche mit genutzten Probebäumen in Abhängigkeit von der Eigentumsart und dem Vorrat/ ha

Mittels logistischer Regressionsanalyse wurde ein Modell entwickelt, welches die Wahrscheinlichkeit des Ausscheidens von Probebäumen in Abhängigkeit von verschiedenen Faktoren schätzt. Hierbei wurde die Wahrscheinlichkeit der Nutzung von Probebäumen in Abhängigkeit von den im BWI-Datensatz enthaltenen Parametern getestet. Somit konnte lediglich ein Teil der in Abbildung 29 aufgeführten möglichen Einflussfaktoren Bestandteil des Modells werden. Hierzu gehörten:

1.) Lageparameter der Traktecke:

- $\quad$ Eigentumsart (Staatswald = Bundes- und Landeswald, Körperschaftswald, Privatwald)

- Der Privatwald wurde in vier Betriebsgrößenklassen unterteilt:

1. bis 20 ha

2. 21 bis 200 ha

3. 201 bis 1.000 ha

4. größer 1.000 ha

- Wuchsgebiet (Niedersächsisches Tiefland, Niedersächsisches Bergland) 
- Höhe über NN (m)

- Geländeneigung (Grad)

- Zerschneidung (Anzahl Waldaußen- und -innenränder)

- Erschließung (Rücke- und Fahrwege befestigt und unbefestigt vorhanden bzw. nicht vorhanden)

2.) Parameter des aufstockenden Bestandes:

- Mittleres Bestandesalter (Jahre)

(Das mittlere Bestandesalter und der mittlere BHD wurden als mit den Grundflächen gewogener Mittelwert der Einzelbaumangabe berechnet.)

- Mittlerer BHD (cm)

- Vorrat/ha (Vfm)

- Grundfläche/ha (Vfm)

- Volumenanteil der Hauptbaumart

Es wurde für jeden Bestandestyp die Wahrscheinlichkeit pro Traktecke der 1. Bundeswaldinventur berechnet, dass bis 2002 ein oder mehrere Probebäume genutzt wurden. In Niedersachsen lag die Stichprobenverteilung der BWI je nach Region in einem 4 x 4 km bzw. 2,83 x 2,83 km Netz. Die dadurch bedingte unterschiedliche Repräsentativität der Traktecken fand im Modell durch eine Gewichtung der Traktecken mit dem jeweiligen Stichprobennetz Beachtung. Zur Berechnung der Wahrscheinlichkeit des Ausscheidens von Probebäumen auf einer Traktecke wurden nur jene der oben aufgeführten Variablen ins Modell aufgenommen, deren Koeffizienten signifikant verschieden (95\%-Niveau) von Null waren und die einen wesentlichen Erklärungsbeitrag leisten konnten.

Tabelle 8 zeigt die Koeffizienten der signifikanten Einflussfaktoren auf die Nutzung. Hierbei wird die Wahrscheinlichkeit für die Nutzung eines Probebaums in Abhängigkeit zum Bestandestyp deutlich, wobei der Bestandestyp Buche als Vergleichsbasis dient. Die Wahrscheinlichkeit des Ausscheidens eines Probebaumes sinkt damit im Bestandestyp Eiche im Vergleich zum Bestandestyp Buche um den Faktor 0,753. Im Bestandestyp Laubholz niedrigen Umtriebs beträgt der Faktor 0,693. Im Bestandestyp Fichte steigt die Wahrscheinlichkeit im Vergleich zum Bestandestyp Buche um den Faktor 1,941, im Bestandestyp Kiefer steigt sie um den Faktor 1,734. Ebenfalls so zu verstehen sind die Zeilen 7-12 der Tabelle 8, die den Einfluss der Eigentumsarten auf die Nutzung darstellen. Hier wurden die verschiedenen Eigentumsarten mit dem Staatswald ins Verhältnis gesetzt.

Nominal skaliert hingegen sind die Variablen Wuchsgebiet „Bergland“, „Erschließung vorhanden“ und „Zerschneidungen“. Sollte somit bei einem Bestand die Erschließung vorhanden sein bzw. dieser zudem im Bergland liegen, so erhöht sich die Wahrscheinlichkeit der Nutzung um die Faktoren 1,379 bzw. 1,401 . 
Die Nutzungswahrscheinlichkeit sinkt bei der Variablen „Geländeneigung“ mit dem Faktor 0,99 bei einer zusätzlichen Geländeneigung um ein Grad bzw. steigt mit dem Faktor 1,007, sollte der „Vorrat/ha“ um einen Vfm steigen. Zwar sind beiden Faktoren nur gering von 1,0 verschieden, jedoch haben sie durch die breite Spannweite der Variablenwerte den höchsten Einfluss auf die Nutzungswahrscheinlichkeit.

Tabelle 8: Ergebnistabelle der logistischen Regression für die untersuchten Nutzungs-Einflussfaktoren (Gesamtmodell) ( ${ }^{*}=$ nicht signifikant)

\begin{tabular}{|c|c|c|}
\hline Skalatyp & Variable & Faktor \\
\hline \multirow[t]{5}{*}{ Verhältnisskala } & Bestandestyp Buche & 1,000 \\
\hline & Bestandestyp Eiche & 0,753 \\
\hline & Bestandestyp Aln & 0,693 \\
\hline & Bestandestyp Fichte & 1,941 \\
\hline & Bestandestyp Kiefer & 1,734 \\
\hline \multirow[t]{6}{*}{ Verhältnisskala } & Staatswald & 1,000 \\
\hline & Privatwald bis 20 ha & 0,371 \\
\hline & Privatwald $21-200$ ha & 0,632 \\
\hline & Privatwald 201-1.000 ha & $1,002^{*}$ \\
\hline & Privatwald $>1.000$ ha & 0,861 \\
\hline & Körperschaftswald & 0,621 \\
\hline \multirow[t]{3}{*}{ Nominalskala } & Wuchsgebiet Bergland & 1,401 \\
\hline & Erschließung vorhanden & 1,379 \\
\hline & Zerschneidung & 0,751 \\
\hline \multirow[t]{3}{*}{ Intervallskala } & Geländeneigung (Grad) & 0,991 \\
\hline & Vorrat/ha & 1,007 \\
\hline & Konstante & 0,256 \\
\hline
\end{tabular}


Für die Bestandestypen wurden zudem noch einzelne Modelle gerechnet, so dass für diese deren Abhängigkeit von bestimmten Einflussfaktoren deutlich wird (vgl. Tab. 9). Die darin genutzten Variablen wurden wie in der vorangegangen Tabelle skaliert. Der Übersichtlichkeit wegen wurde die Darstellung auf Vorzeichen begrenzt, um lediglich die Richtungswirkung zu verdeutlichen. Felder ohne Vorzeichen konnte auf der Basis des vorliegenden Datensatzes nicht berechnet werden.

Tabelle 9: $\quad$ Signifikante Einflusskoeffizienten und deren Wirkungsrichtung in Abhängigkeit vom Bestandestyp (* = nicht signifikant)

\begin{tabular}{|c|c|c|c|c|c|c|}
\hline \multirow[t]{2}{*}{ Skalatyp } & \multirow[t]{2}{*}{ Variable } & \multicolumn{5}{|c|}{ Bestandestyp } \\
\hline & & Buche & Eiche & ALn & Fichte & Kiefer \\
\hline Verhältnisskala & $\begin{array}{l}\text { Privatwald bis } 20 \text { ha } \\
\text { Privatwald } 21-200 \text { ha } \\
\text { Privatwald } 201-1.000 \text { ha } \\
\text { Privatwald }>1.000 \mathrm{ha} \\
\text { Körperschaftswald }\end{array}$ & $\begin{array}{l}-- \\
- \\
+ \\
++ \\
-\end{array}$ & $\begin{array}{l}-- \\
-- \\
- \\
- \\
-\end{array}$ & $\begin{array}{l}- \\
- \\
- \\
- \\
- \\
-\end{array}$ & $\begin{array}{l}- \\
- \\
- \\
- \\
++ \\
--\end{array}$ & $\begin{array}{l}-- \\
- \\
+ \\
- \\
-\end{array}$ \\
\hline Nominalskala & $\begin{array}{l}\text { Wuchsgebiet Bergland } \\
\text { Erschließung/Wege } \\
\text { Zerschneidung/ } \\
\text { Waldrand } \\
\text { Alter }<40\end{array}$ & $\begin{array}{l}++ \\
++ \\
-- \\
-\end{array}$ & $\begin{array}{c}+ \\
++ \\
-\end{array}$ & ++ & ++ & + \\
\hline Intervallskala & $\begin{array}{l}\text { Vorrat/ha } \\
\text { Geländeneigung }\end{array}$ & + & + & + & + & + \\
\hline
\end{tabular}

Zur Erklärung der Tabelle 9 sei beispielsweise der Bestandestyp Buche herangezogen: Liegt ein Bestand mit vorherrschender Buche im Bergland, so erhöht sich die Wahrscheinlichkeit, dass ein Probebaum genutzt wird, deutlich $(++)$. Stockt der Bestand im Privatwald der Größenklasse bis 20 ha, sinkt die Wahrscheinlichkeit einer Nutzung deutlich (- -), im Privatwald der Betriebsgrößenklasse 2011000 ha hingegen steigt die Wahrscheinlichkeit leicht an (+). Steigt die Geländeneigung einer Fläche um eine Einheit (Grad), dann sinkt die Wahrscheinlichkeit der Nutzung leicht (-). Auf einer von befestigten oder unbefestigten Wegen erschlossenen Fläche wird mit einer deutlich höheren Wahrscheinlichkeit $(++)$ ein Probebaum genutzt als auf einer unerschlossenen Fläche. Jeder zusätzliche Waldrand, der einen Bestand zerschneidet, senkt die Wahrscheinlichkeit, dass am Ende des Betrachtungszeitraumes ein Probebaum auf einer Traktecke fehlt, deutlich (- -). 
Steigt der Hektarvorrat eines Bestandes, dann erhöht sich die Nutzungswahrscheinlichkeit $(+)$. Ist der Bestand jünger als 40 Jahre dann sinkt die Wahrscheinlichkeit (-).

Anschließend wurde zur Verifizierung des Modells bei allen Traktecken mit einer vom Modell errechneten Wahrscheinlichkeit von mehr als $50 \%$ unterstellt, dass ein Probebaum zwischen 1987 und 2002 geerntet wurde. Damit konnte überprüft werden, welche Traktecken nach dem Modell richtig zugeordnet wurden. Die Anteile richtig zugeordneter Traktecken zeigt Tabelle 10; sie können als grundsätzliche Bestätigung des gewählten Modellansatzes interpretiert werden.

Tabelle 10: Anteil der nach dem logistischen Regressionsmodell richtig zugeordneten Traktecken pro Bestandestyp

\begin{tabular}{c|c}
\hline Bestandestyp & $\begin{array}{c}\text { Anteil richtig zugeordneter } \\
\text { Traktecken }\end{array}$ \\
\hline Buche & $73 \%$ \\
Eiche & $73 \%$ \\
ALn & $81 \%$ \\
Fichte & $83 \%$ \\
Kiefer & $69 \%$ \\
\hline
\end{tabular}

Das modellierte Nutzungsverhalten der Waldeigentümer erscheint auch rational. Da in Beständen mit führendem Nadelholz höhere Deckungsbeiträge bei einer Holznutzung unterstellt werden können, ist die Wahrscheinlichkeit, dass ein Probebaum ausscheidet, höher als in Beständen mit führendem Laubholz. Wobei die Wahrscheinlichkeit im Bestandestyp Fichte am höchsten ist und über die Bestandestypen Kiefer, Buche, Eiche und Laubholz niedrigen Umtriebs sinkt (vgl. Tab. 8). Höhere Holzvorräte auf einer Fläche geben einen höheren Anreiz zur Holznutzung, weil der mögliche Ertrag steigt. Großprivatwaldbesitzer und der Staatswald verfügen aufgrund ihrer Flächengröße im Vergleich zum Kleinst- und Kleinprivatwald in der Regel über eigene Betriebs- und Vermarktungsorganisationen und haben deshalb geringere Holzernte- und Vermarktungskosten. Hinzu kommt, dass mit steigender Betriebsgröße der Wald in der Regel zunehmend als Lebensgrundlage des Waldbesitzers dient und von daher allein schon die Bewirtschaftungswahrscheinlichkeit steigt. Die Nutzungswahrscheinlichkeit dürfte im Niedersächsischen Bergland deshalb höher sein, weil sich hier die produktiveren Waldflächen konzentrieren. Auch mit Blick auf die technische Realisierbarkeit der Holzernte ist das Nutzungsverhalten plausibel, da, je geringer das Gefälle, je weniger zerschnitten und je besser erschlossen das Gelände ist, desto geringer sind der technische Aufwand und die Holzerntekosten. 
Das Modell erklärt, welche Einflussfaktoren die Mobilisierung von Holz in der Vergangenheit eingeschränkt haben. Da sich beispielsweise insbesondere im Kleinprivatwald des niedersächsischen Flachlands häufig die als nutzungseinschränkend identifizierten Variablen Zerschneidung, schlechte Erschließung und geringe Hektarvorräte ballen, fanden hier auch nur wenige Holznutzungen statt. Nach dem Modell wird sich jedoch zukünftig die Nutzungswahrscheinlichkeit vieler Flächen schon deshalb erhöhen, weil der Vorrat seit 1987 angestiegen ist. Die weiteren relevanten Einflussgrößen auf die Nutzungswahrscheinlichkeit machen zudem deutlich, an welchen Punkten die Politik ansetzen muss, um bisher nicht genutzte Holzvorräte zu mobilisieren.

$\mathrm{Zu}$ bedenken ist hier jedoch auch, dass sich die Analyse nur auf den Zeitraum 1987 bis 2002 bezieht und das mittlere Nutzungsverhalten nur während dieses Zeitintervalls analysiert wurde. Änderungen des Nutzungsverhaltens seit Abschluss der BWI 2, die im Zusammenhang mit den jüngsten Erhöhungen der Holzverarbeitungskapazitäten und entsprechenden Preiserhöhungen zunehmend diskutiert werden, sind durch den retrospektiven Analyseansatz nicht erfassbar. 



\section{Forstliche Nutzungspotenziale}

\subsection{Material und Methoden}

Die Schätzung der forstlichen Nutzungspotenziale für Niedersachsen basiert auf mehreren computergestützten Szenariosimulationen. Der Prognosebeginn wurde auf das Jahr 2007 festgelegt und die Prognosedauer beträgt insgesamt dreißig Jahre, wobei Zwischenergebnisse in Fünfjahresschritten ausgegeben werden. Dabei wird der Begriff „forstliches Nutzungspotenzial“ als die Holzmenge verstanden, welche unter strikter Einhaltung der Szenariovorgaben im Rahmen der simulierten forstlichen Eingriffe (Vor- und Endnutzung) anfällt. Ein eigentümerspezifisches Nutzungsverhalten wird gesondert nur für die ersten fünfzehn Jahre des Prognosezeitraums berücksichtigt. Die Datengrundlage für die Bestimmung des Ausgangszustands (Status-quo) bildeten die niedersächsischen Einzelbaumerhebungen der zweiten Bundeswaldinventur (BWI 2), die Holzeinschlagsstatistik und die Schadenserhebungen des Sturms Kyrill (vgl. Abb. 32).

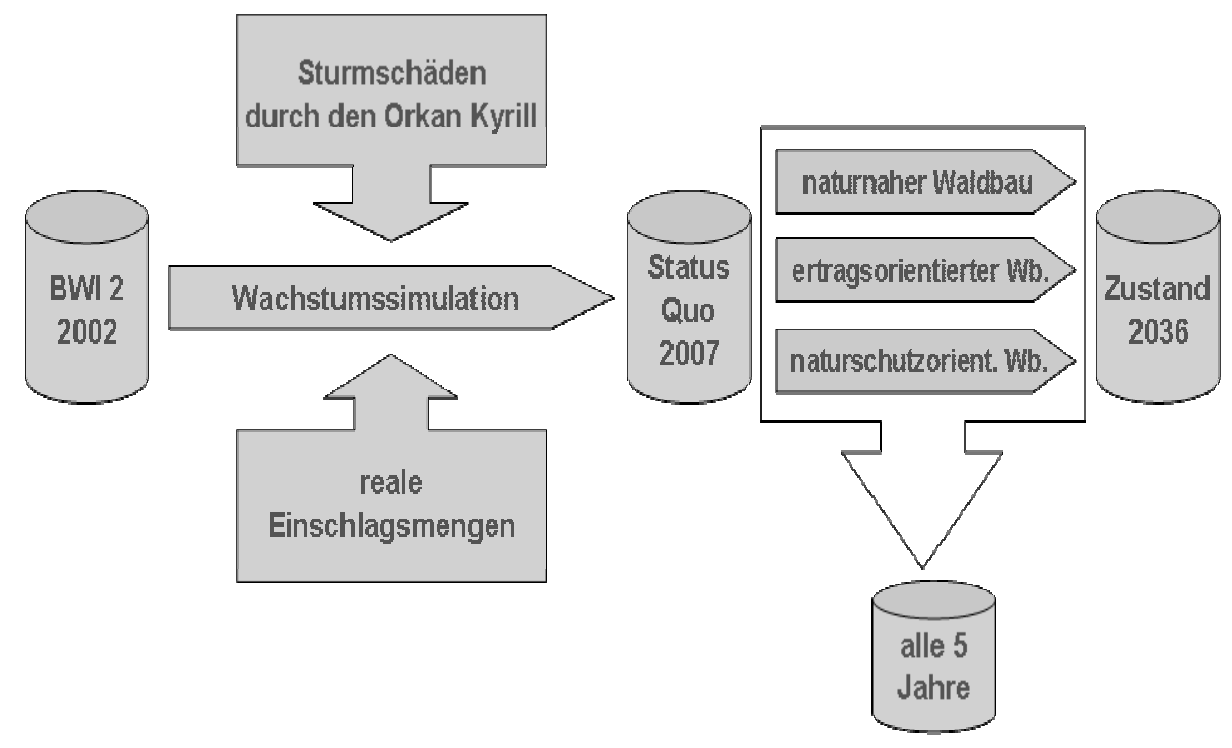

Abbildung 32: Schematischer Aufbau des Gesamtprognosesystems 


\subsubsection{Vorstellung der Prognosewerkezenge}

Für die Simulation der unterschiedlichen Szenarien wurde das Softwaresystem „WaldPlaner“ eingesetzt. Es basiert auf dem Einzelbaumwuchsmodell BWINPro (NAGEL 2005) und wurde zur Simulation und Auswertung einer großen Anzahl von einzelnen Beständen weiterentwickelt. Das Programm unterstützt das Generieren virtueller Modellbestände aus Forsteinrichtungsdaten oder Einzelbauminventuren und die Datenhaltung in einer beliebigen Datenbank. Die virtuellen Bestände werden einzelbaumweise aufgebaut und simuliert. Das System besteht aus mehreren Komponenten:

- Datenbankschnittstelle

- Datenergänzung

- Einzelbaumwachstumssimulator

- Sortierroutine

- Szenariomanager

- Auswertungstool

Die Komponenten Datenergänzung, Einzelbaumwuchsmodell und die Sortierroutine sind dem Softwarepaket TreeGrOSS (NAGEL et. al. 2006, siehe Glossar) entnommen. Der Szenariomanager greift auf eine ebenfalls in JAVA realisierte Komponente (DUDA 2006) zurück, die über eine Vielzahl waldbaulicher Handlungsalternativen (Z-Baumauswahl, Durchforstung, Endnutzung, Pflanzung usw.) gesteuert wird. Diese einzelnen Elemente wurden im Szenariomanagement zu einem Gesamtkonzept vereinigt, das Szenariosimulationen ermöglicht, die über die Einsteuerung unterschiedlicher Waldbauvarianten flexibel gestaltet werden können.

\subsubsection{Startwerte 2007}

Der Stichtag dieser Studie wurde auf den 01.01 .07 festgelegt. Um eine geeignete Datenbasis zur Simulation der einzelnen Strategien zu erhalten, wurden zunächst auf Basis der Einzelbaumerhebungen der zweiten Bundeswaldinventur virtuelle (Einzelbaum-) Bestände für das Jahr 2002 generiert. Da das Wuchsmodell Bäume ab einem Brusthöhendurchmesser von $7 \mathrm{~cm}$ verarbeitet, wurden die Bestände nur aus Bäumen erstellt, die diese Schwelle überschreiten. Bäume mit einem geringeren Durchmesser wurden der Verjüngung zugerechnet, welche ebenfalls kumulativ als Schicht (mittlere Höhe, Alter und Pflanzenanzahl) Eingang in das Wuchsmodell findet und bei der Simulation des Einwuchses (Bäume, die $7 \mathrm{~cm}$ BHD überschreiten) zum Tragen kommt. Die Modellbestände weisen eine kreisförmige Fläche mit einer Größe von 0,2 ha auf. Die Modellbestandsgröße wurde so gewählt, weil bei zu kleinen Beständen virtuelle Bestandesbehandlungen kaum oder gar nicht sinnvoll umzusetzen sind, andererseits mit zunehmender Modellbestands- 
größe auch der Rechen- und Speicherbedarf erheblich steigt, da die Mortalität und die Nutzungen positionsabhängig modelliert werden.

Das Aufnahmedesign der zweiten Bundeswaldinventur basiert auf einem systematischen Stichprobennetz mit einer Basisgitterweite von $4 \mathrm{~km}$ x $4 \mathrm{~km}$, welches in Niedersachsen in waldarmen Regionen auf $2,83 \mathrm{~km}$ x 2,83 $\mathrm{km}$ verdichtet wurde. An jedem Gitterpunkt wurde ein Quadrat bzw. Trakt mit einer Seitenlänge von $150 \mathrm{~m}$ in nordöstliche Richtung aufgespannt. Fällt eine Ecke des Traktes in eine Waldfläche laut Definition, wurden neben einer Vielzahl weiterer Erhebungen u. a. Bäume mittels einer Winkelzählprobe erfasst und mehrere Einzelbaumparameter (Brusthöhendurchmesser, Höhe, Position, ...) gemessen. In Niedersachsen wurden insgesamt 4.615 Trakte ausgewählt, von denen 1.501 Waldtrakte sind. Aufbauend auf den Erhebungen an den einzelnen Traktecken wurden insgesamt 3621 Modellbestände für die weitere Simulation und Auswertung generiert (vgl. Abb. 33). Diese repräsentieren den Waldzustand im Jahr 2002 und bilden ihn modellhaft nach.

Der Unterschied in der Anzahl der Trakte, die der Analyse des Nutzungsverhaltens (s. Kap. 3) und der Holzaufkommensprognose zugrunde liegt, erklärt sich durch Waldflächenveränderungen zwischen der BWI 1 und der BWI 2 sowie durch den Umstand, dass bei der Potenzialabschätzung auch die Trakte mit Nutzungsverboten und lediglich einer Verjüngungsschicht in die Simulationsrechnungen mit einbezogen wurden (s. Kap. 4.2.5).

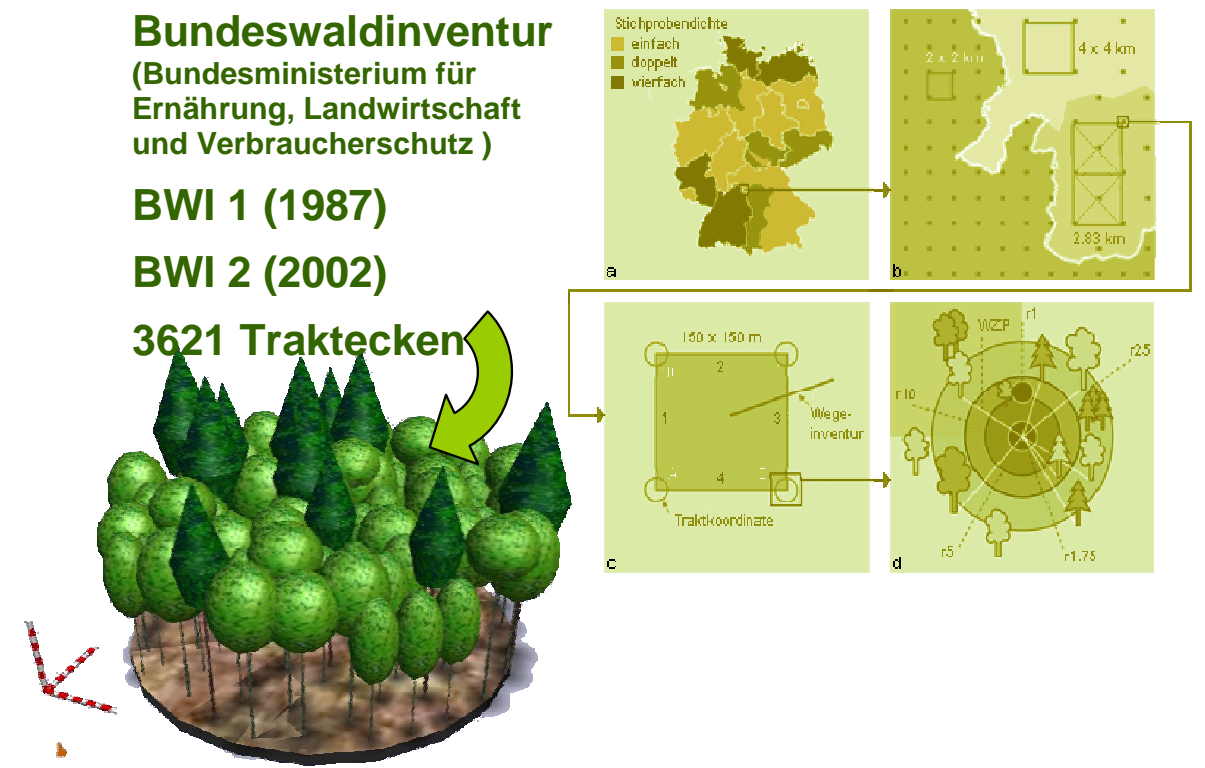

Abbildung 33: Aufnahmedesign der zweiten Bundeswaldinventur (rechts; BMVEL 2004) und ein aus den Einzelbaumerhebungen einer Traktecke resultierender Modellbestand (links) 
Der Ausgangszustand 2002 wurde in einem zweiten Schritt in das Jahr 2006 überführt. Dazu wurden die Modellbestände unter Berücksichtigung der nach Eigentumsart getrennt vorliegenden Einschlagsmengen (Holzeinschlagsstatistik) vier Jahre mit dem WaldPlaner fortgeschrieben. Zur Berücksichtigung der Schadensmeldungen nach dem Sturm „Kyrill“ am 18.01.2007 in der ersten Prognoseperiode wurden die auf Forstamts- bzw. Forstbetriebsebene gemeldeten Sturmholzmengen mit ihren Dimensionsschwerpunkten zufällig in den entsprechenden Straten der vorgegebenen Gebiete iterativ „entnommen“. Dabei wurden ausgewählte Modellbestände komplett „abgeräumt“. Nester- oder Einzelstammwürfe wurden nicht modellhaft umgesetzt. Insgesamt wurden sieben Sturmschadensregionen ausgewiesen: Nordhorn/Bentheim, Melle/Osnabrück, Celle, Weserbergland/Hils, Solling/Bramwald, Harzvorland und Harz. Die Schwerpunkte des Sturmschadens lagen im Niedersächsischen Bergland und im Süden des Tieflands, in denen insgesamt ca. 2,5 Mio. Vfm Sturmholz angefallen sind, wobei die Hauptmasse auf die Nadelhölzer entfiel (vgl. Abb. 34).

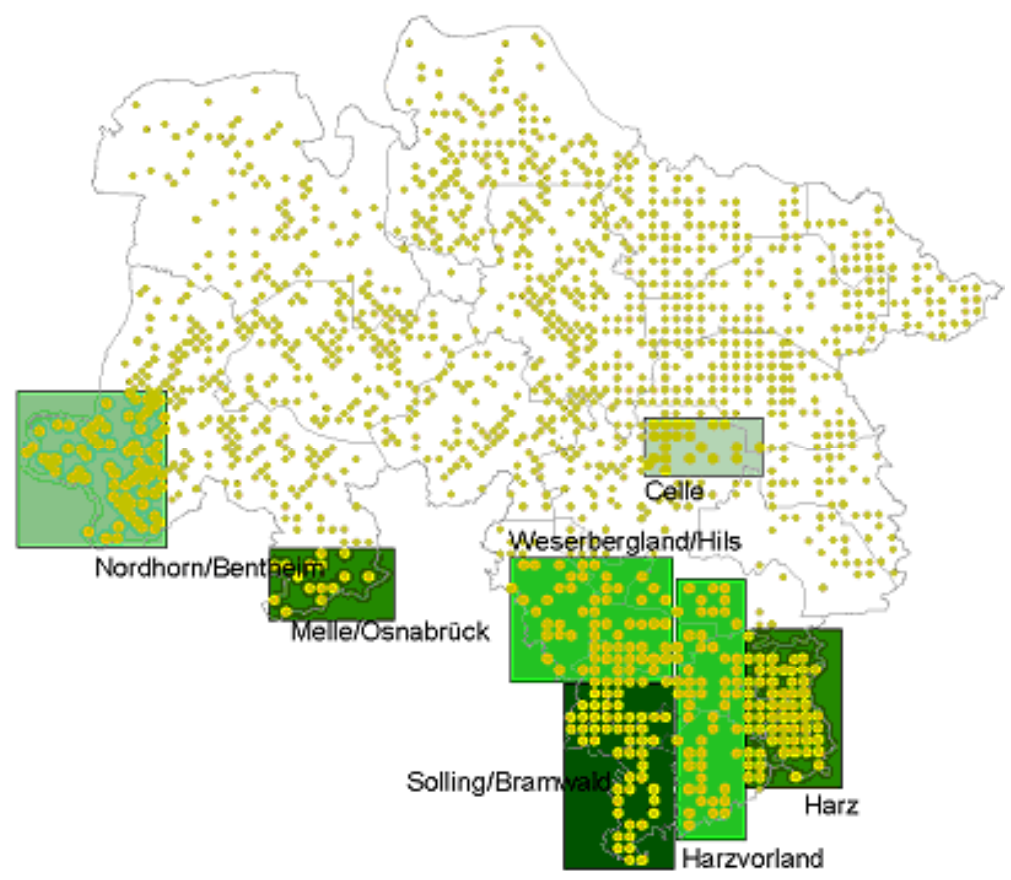

Abbildung 34: Die Trakte der BWI 2 und die Hauptschadensregionen des Sturms Kyrill; je dunkler die Schadensregion, desto böher der Sturmschaden 


\subsubsection{Waldentwicklungsszenarien}

Im Rahmen der Simulation wurden drei Waldentwicklungsszenarien umgesetzt:

- „naturnaher“ Waldbau

- „ertragsorientierter“"Waldbau

- „naturschutzorientierter“ Waldbau

Die Szenarien werden über naturschutzorientierte Parameter (Minderheitenschutz, Habitatbaumanzahl, Totholzvolumen) und eingriffsspezifische Parameter (Eingriffsturnus, Zielstärken, Endnutzungsmassen, Vornutzungsmassen, Durchforstungsbeginn, Freistellung) eingesteuert und sollen einen breiten Fächer möglicher Waldbauoptionen abdecken (vgl. Tab. 11). Die Spanne der zu schützenden Habitatbäume reicht z. B. von 0 („ertragsorientiert"), über 3 („,naturnah“) bis zu 10 (,naturschutzorientiert“) Bäume je Hektar.

Tabelle 11: Rabmenwerte der drei gerechneten Szenarien „naturnab“, „naturschutzorientiert" und „ertragsorientiert"

\begin{tabular}{|c|c|c|c|}
\hline „Stellschrauben“ & $\begin{array}{l}\text { „ertrags- } \\
\text { orientiert }^{66}\end{array}$ & „naturnah“ & $\begin{array}{l}\text { "naturschutz- } \\
\text { orientiert" }\end{array}$ \\
\hline Schutz seltener Baumarten & nein & ja & ja \\
\hline Habitatbäume (n/ha) & 0 & 3 & 10 \\
\hline Totholz $\left(\mathrm{m}^{3} / \mathrm{ha}\right)$ & bis 10 & 20 & 40 \\
\hline Zielstärke (cm) & $\begin{array}{l}\text { Ei } 65 \text {, Bu 55, } \\
\text { Fi } 40, \text { Ki } 40\end{array}$ & $\begin{array}{l}\text { Ei 70, Bu 60, } \\
\text { Fi 45, Ki } 45\end{array}$ & $\begin{array}{l}\text { Ei } 75, \mathrm{Bu} 65 \\
\text { Fi } 50, \text { Ki } 50\end{array}$ \\
\hline $\begin{array}{l}\text { Endnutzungsmasse pro Hieb } \\
\left(\mathrm{m}^{3} / \mathrm{ha}\right)\end{array}$ & $\begin{array}{l}\text { Min. } 10-15 \\
\text { Max. } 70-115\end{array}$ & $\begin{array}{l}\text { Min. } 10-15 \\
\text { Max. } 70-115\end{array}$ & $\begin{array}{l}\text { Min. } 10-15 \\
\text { Max. 60-120 }\end{array}$ \\
\hline $\begin{array}{l}\text { Durchforstungsbeginn } \\
\text { Bestandesoberhöhe (m) }\end{array}$ & $12-18$ & $10-16$ & $10-16$ \\
\hline $\begin{array}{l}\text { Durchforstungsmasse pro Hieb } \\
\left(\mathrm{m}^{3} / \mathrm{ha}\right)\end{array}$ & $25-110$ & $25-100$ & $25-110$ \\
\hline Freistellungsgrad der Z-Bäume & sehr stark & stark & stark \\
\hline
\end{tabular}

Habitatbäume werden der Nutzung entzogen und dem natürlichen Wachstum und Zerfall überlassen. Auf diese Weise bilden sie somit die Lebensgrundlage für viele Organismen. Vor einem ähnlichen ökologischen Hintergrund ist die Menge des im Wald belassenen Totholzes zu sehen. In der „,naturschutzorientierten“ Variante werden $40 \mathrm{~m}^{3}$ Totholz je Hektar angestrebt, in der ,naturnahen“ Variante reduziert sich diese Menge auf die Hälfte und im Rahmen der „ertragsorientierten“ Variante werden bis zu $10 \mathrm{~m}^{3}$ angesteuert. 
Die Zielstärke bezeichnet baumartenspezifisch den Brusthöhendurchmesser eines Baumes, ab welchem endgenutzt werden sollte. Ausgehend von den Vorgaben der „naturnahen“ Variante wurde im „ertragsorientierten“ Waldbauszenario die Zielstärke jeweils um $5 \mathrm{~cm}$ abgesenkt, im „naturschutzorientierten“ Waldbau um $5 \mathrm{~cm}$ erhöht. Die Spanne der Zielstärken reicht somit von $75 \mathrm{~cm}$ BHD für die Eiche (,naturschutzorientiert") bis zu $40 \mathrm{~cm}$ BHD bei Kiefer (,ertragsorientiert"). Die Durchforstungen werden in der „ertragsorientierten“ Variante später und dafür stärker durchgeführt. Der Durchforstungsbeginn wird über die Bestandesoberhöhe eingesteuert. Diese liegt bei Durchforstungsbeginn in der „ertragsorientierten" Variante im Mittel um $2 \mathrm{~m}$ höher als bei den anderen beiden Varianten. In den Endnutzungsmassen pro Hieb unterscheiden sich die Szenarien nur unwesentlich, weil deren Höhe primär von den damit verbundenen Flächen und Risiken bestimmt wird.

\subsubsection{Sortierungsvorgaben}

Bei der Aushaltung, d. h. bei der Zerlegung der gefällten Bäume in einzelne, dem Verwendungszweck entsprechende Sortimente, wurde grundsätzlich nach Laubund Nadelholz differenziert. Laubstammholz wurde lang und in Abschnitten ausgehalten, Nadelholz nur in Abschnitten. Abschnitte unterscheiden sich vom Langholz dahingehend, dass die Längen der aus einem Stamm herausgeschnittenen Teilstücke festgelegt sind. Der Mindestzopfdurchmesser beträgt für Laubholz $21 \mathrm{~cm}$, für Nadelholz $12 \mathrm{~cm}$. Bei der Sortierung des Laubholzes wurde zusätzlich die Beschränkung eingeführt, dass das Stammholz nur bis zum Kronenansatz ausgehalten wurde und mindestens $5 \mathrm{~m}$ lang sein musste. Bei der Aushaltung des Nadelholzes wurde die Abschnittslänge auf $5 \mathrm{~m}$ festgelegt. Die Stockhöhe betrug bei allen Baumarten $30 \mathrm{~cm}$. Die gebildeten Sortimente werden nach ihrer Mittenstärke klassiert, so dass zusätzlich zur Holzmenge auch dimensionsabhängige Informationen über das Rohholz vorliegen.

Eine Qualitätssortierung wurde nicht vorgenommen, da die entsprechenden Informationen im niedersächsischen Datensatz der BWI 2 nicht enthalten sind und derzeit marktbedingte Substitutionsprozesse zwischen den traditionellen verwendungsorientierten Sortimenten (Palette, Industrieholz, Brennholz usw.) ablaufen. 
4.2 Forstliche Nutzungspotenziale in Niedersachsen bis 2036 bei unterschiedlichem Nutzungsverhalten

\subsubsection{Entwicklung der Holzvorräte}

Als Holzvorrat wird hier die gesamte oberirdische Derbholzmasse (über $7 \mathrm{~cm}$ BHD) eines Bestandes oder einer Summe von Beständen verstanden (Vorratsfestmeter, Vfm $\left[\mathrm{m}^{3}\right.$ oder $\left.\left.\mathrm{m}^{3} / \mathrm{ha}\right]\right)$. Der Vorrat und dessen zeitliche Entwicklung sind mit die wichtigsten Indikatoren zur Beurteilung forstwirtschaftlicher Betriebe. Die Zusammensetzung des Vorrates (Baumartenanteile, Altersverteilung, Durchmesserverteilung usw.), seine Gesamthöhe und seine Entwicklung ermöglichen die Beurteilung ökonomischer und ökologischer Potenziale unter dem Aspekt einer nachhaltigen Forstwirtschaft.

Abbildung 35 zeigt die prognostizierte Vorratsentwicklung in Niedersachsen in den Jahren von 2002 bis 2036.

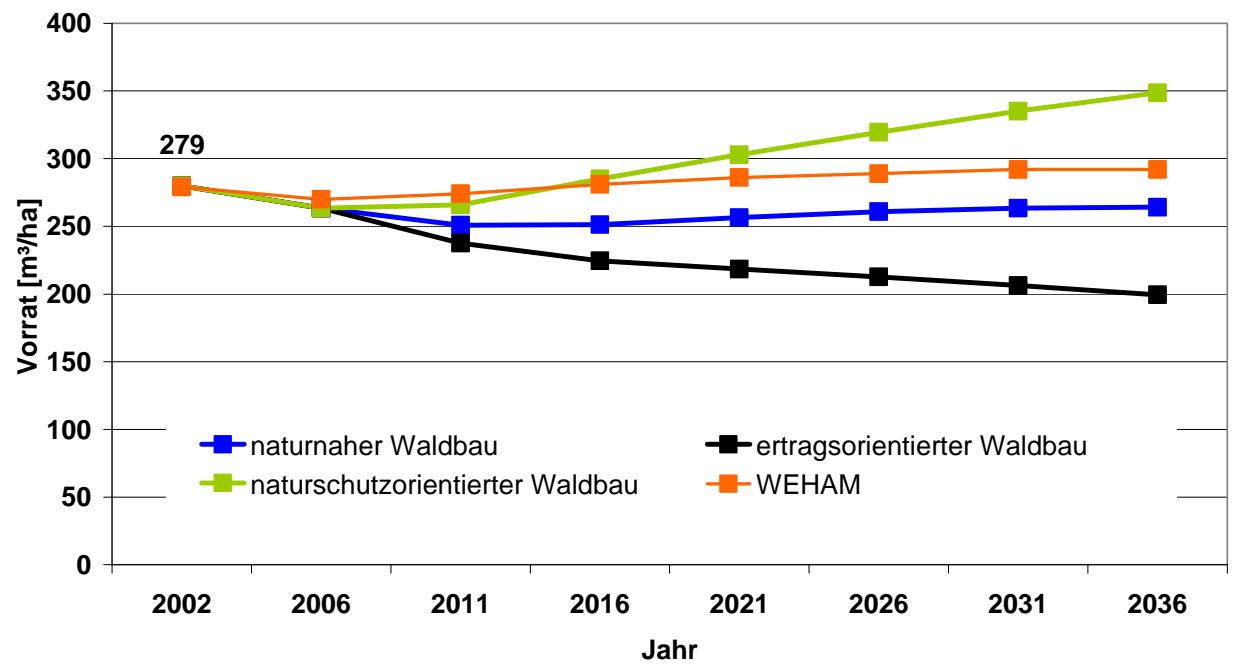

Abbildung 35: Entwicklung der flächenbezogenen Vorräte der 3 Szenarien im Vergleich zum Basisszenario nach WEHAM

Der Vorrat im Jahr 2002 (ca. 279 Vfm/ha) basiert auf den Einzelbaumerhebungen der zweiten Bundeswaldinventur, wobei das Baumvolumen mittels der im Prognosesystem implementierten Volumenfunktionen hergeleitet wurde. Von diesem Startwert ausgehend ist zunächst bis zum Jahr 2006 bei allen Szenarien ein Vorratsabbau zu verzeichnen. Das Basisszenario nach WEHAM (s. Glossar) weist danach einen leichten Vorratsaufbau auf. Der Holzvorrat steigt bis zum Jahr 2036 auf $292 \mathrm{Vfm} / \mathrm{ha}$. Ein vergleichbaren Trend auf geringerem Niveau zeigt sich 
beim Szenario „naturnaher Waldbau“. Dieses Szenario führt im Jahr 2036 zu einem Vorrat von $264 \mathrm{Vfm} / \mathrm{ha}$. Der stärkste Vorratsaufbau wird im Szenario „,naturschutzorientierter Waldbau“ generiert. Bedingt durch eine verzögerte Holznutzung, geringere Nutzungsmassen und einen verstärkten Habitatbaumschutz liegt hier der Vorrat am Ende der Simulationsperiode bei $348 \mathrm{Vfm} / \mathrm{ha}$. Das Szenario „ertragsorientierter Waldbau“ hingegen baut den Vorrat kontinuierlich bis zu einem Wert von $200 \mathrm{Vfm} / \mathrm{ha}$ ab.

Vergleicht man die Durchmesserstruktur der wichtigsten Baumarten im Jahr 2002 und 2036 (vgl. Abb. 36, die WEHAM-Graphen beschreiben die Ausgangszustände 2002), zeigt sich die konsequente, modellhafte Umsetzung der Szenariorahmenwerte.

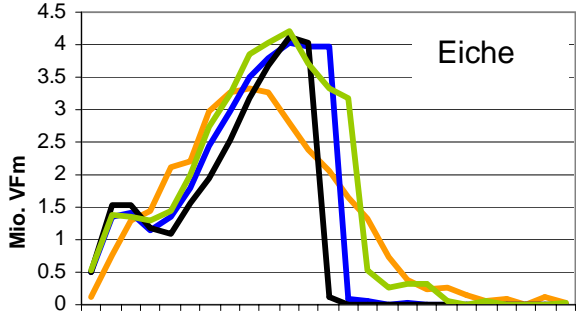

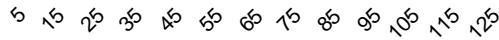

BHD-Klasse

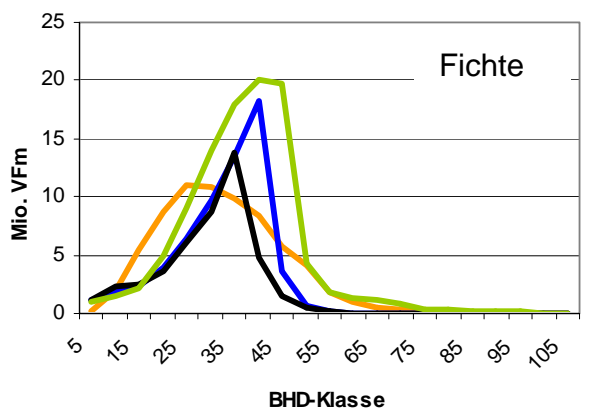

WEHAM
BHD-Klasse

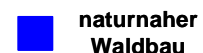

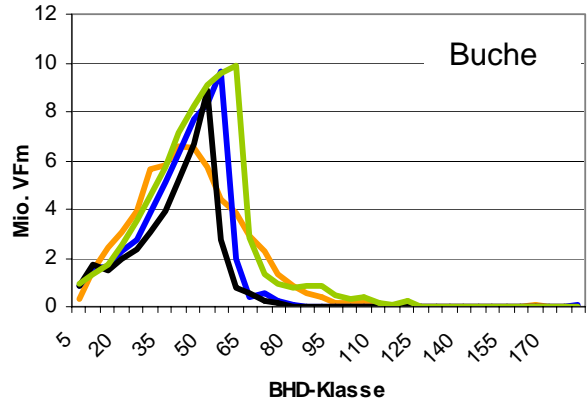

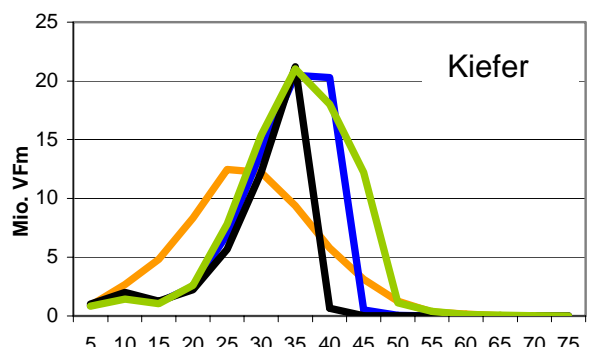

BHD-Klasse

naturschutzorient. Waldbau

Abbildung 36: Holzvorrat über dem Durchmesser für die wichtigsten Baumarten in den Jabren 2002 (WEHAM) und 2036; oben links: Eiche, oben rechts: Buche, unten links Fichte, unten rechts: Kiefer

Die Durchmesserverteilungen im Jahr 2036 fallen deutlich im Bereich der Zielstärke ab. Die symmetrische Struktur verschiebt sich von 2002 bis 2036 dahingehend, dass sich die Masse des Vorrates in den Durchmesserklassen kurz vor Zielstärke befindet. Diese Verschiebung kommt durch die konsequente Ziel- 
stärkennutzung und einer Hiebsruhe zwischen Vor- und Endnutzung zustande. In der Naturschutzvariante sind dennoch auch nennenswerte Holzmassen im Durchmesserbereich oberhalb der Zielstärke vorhanden. Diese werden im Wesentlichen von den der Holzernte entzogenen Habitatbäumen gebildet. Andeutungsweise ist bei Eiche und Kiefer deutlicher als bei Fichte und Buche das Nachwuchspotenzial $z u$ erkennen. Hier zeigt sich in den kleinen Durchmesserklassen ein zweiter Gipfel, der bei einer Verteilung der Durchmesser nach Stammzahl noch deutlicher hervortreten würde.

\subsubsection{Entwicklung der Zuwächse und Nutzungen}

Neben der Entwicklung des Holzvorrates stellen Zuwachs und die Massen des genutzten Holzes zwei weitere wichtige forstliche Kenngrößen dar. Anhand dieser Indikatoren können u. a. die Leistungsfähigkeit der Waldstandorte und die Nachhaltigkeit von Nutzungsstrategien abgeleitet werden. Da im Rahmen einer Simulation waldbaulicher Szenarien von vereinfachten Rahmenbedingungen ausgegangen wird und die Nutzungsmodelle nicht alle realen Restriktionen berücksichtigen, sollte die simulierte genutzte Holzmenge als Potenzial verstanden werden, welches durch die Vielzahl der im Modell nicht zu berücksichtigenden Faktoren in der Regel noch weiter reduziert wird (vgl. Kap. 3).

Das WEHAM-Szenario führt zu einem permanenten Zuwachsrückgang (vgl. Abb. 37). Die jährlichen Zuwächse gehen von 10,6 Vfm/ha*a auf 7,6 Vfm/ha*a zurück. Die Nutzungsmasse im Jahr 2002 ergibt sich direkt aus der zweiten Bundeswaldinventur und beträgt ca. $5 \mathrm{Vfm} /$ ha*a. Die simulierten Nutzungspotenziale verteilen sich homogen über die Prognoseperiode und schwanken zwischen 7,3 und 7,8 Vfm/ha*a. Das „ertragsorientierte“ Szenario weist einen mittleren Zuwachs von 9,6 Vfm/ha*a auf. In der ,naturnahen“ Variante liegt der Zuwachs bei $10 \mathrm{Vfm} / \mathrm{ha*}^{*}$ und in der ,naturschutzorientierten“ Variante bei 10,5 Vfm/ha*a. Bei allen Varianten ist in den beiden letzten Perioden eine fallende Tendenz zu verzeichnen, die im Zusammenhang mit dem Altersaufbau der niedersächsischen Wälder zu sehen ist (vgl. Abb. 2). Die mittleren Nutzungsmassen bis zum Jahr 2006 (4,5 Vfm/ha*a) sind in den drei Szenarien „,naturschutzorientierter Waldbau“, „ertragsorientierter Waldbau“ und „naturnaher Waldbau“ gleich, da sie unter Berücksichtigung tatsächlicher Nutzungsmassen eingespielt wurden. In der darauf folgenden zehnjährigen Periode ist in allen drei Szenarien deutlich der stärkste Holzabgang zu erkennen. Dies ist u. a. dadurch bedingt, dass die Schäden des Sturms Kyrill in diese Periode fallen (2,4 Vfm/ha) und sich die Nutzungsregeln der Szenarien einstellen.

Die mittlere Nutzungsmasse in Erntefestmetern beträgt für die ,naturnahe“ Variante im Durchschnitt 7,2 Efm/ha*a. Die „,ertragsorientierte“ Variante überschreitet diesen Wert mit 8,6 Efm/ha*a. Die geringsten Nutzungsmassen fallen in der „naturschutzorientierten“ Variante mit 4,9 Efm/ha*a an. Das WEHAM- 
Szenario zeigt eine durchschnittliche Nutzung von ca. $6 \mathrm{Efm} / \mathrm{ha*}$ a und liegt zwischen der „naturnahen“ und „naturschutzorientierten“ Variante.
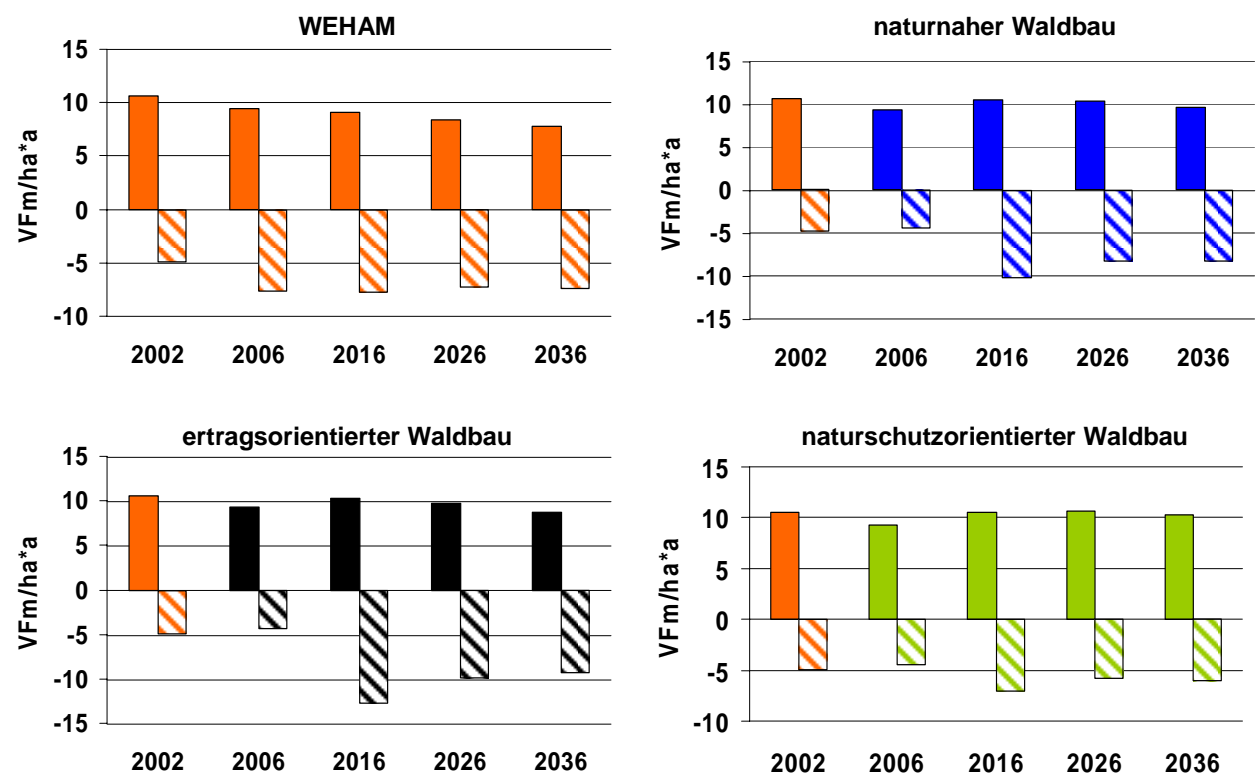

Zuwachs

Nutzung

Abbildung 37: Zuwachs und Nutzung, zum besseren Vergleich in $\mathrm{Vfm} / \mathrm{ha}{ }^{*} a$

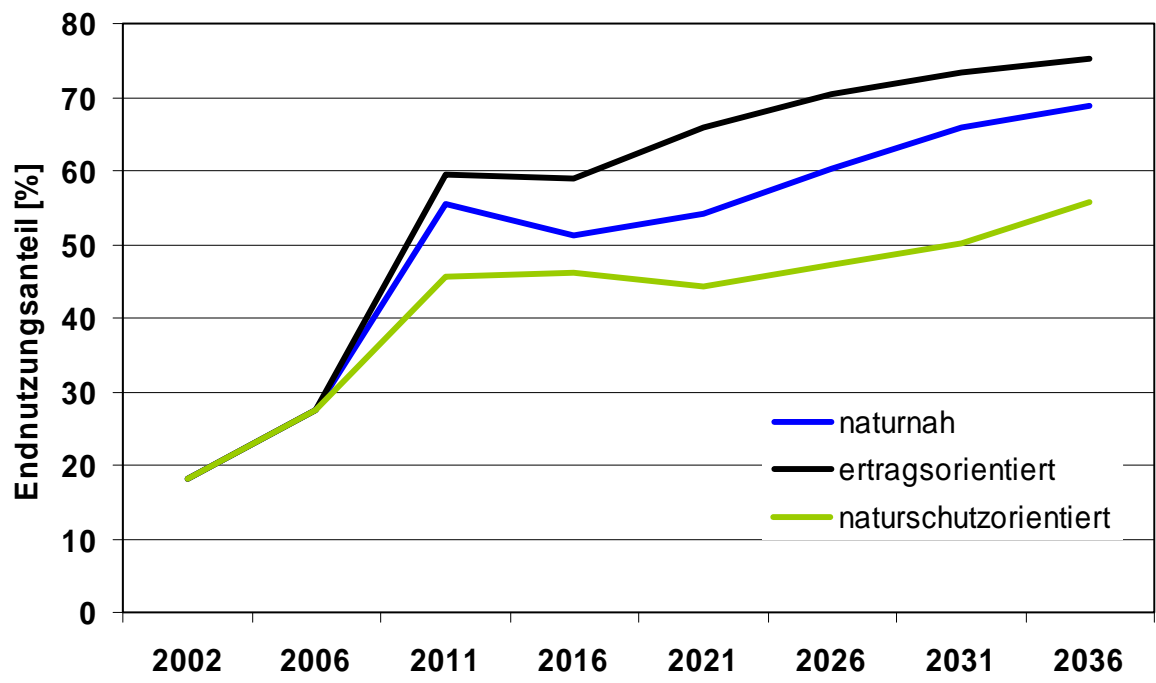

Abbildung 38: Endnutzungsanteile am Nutzungsvolumen 
Die modellhaft entnommenen Nutzungsmengen lassen sich nach Vornutzung und Endnutzung unterteilen. Der Endnutzung werden solche Bäume zugerechnet, die im Rahmen der Zielstärkennutzung anfallen. Abbildung 38 lässt erkennen, dass die Massen aus der Vornutzung deutlich zurückgehen werden. Der Endnutzungsanteil steigt in allen drei Varianten auf 56 bis 75 Prozent an. Dementsprechend werden die Vornutzungserträge stark zurückgehen, so dass weniger schwächere Sortimente anfallen werden. Bis 2006 stammen 70 Prozent des Holzeinschlags aus Vornutzungen. 2036 werden bis zu 75 Prozent der Nutzungsmassen über Endnutzungsmaßnahmen gedeckt. In den Jahren von 2006 bis 2011 steigt der Endnutzungsanteil stark an und geht in der folgenden Periode wieder leicht zurück (Sturmeffekt). In den weiteren Prognoseperioden ist dann ein weniger stark ausgeprägter Anstieg des Endnutzungspotenzials erkennbar.

\subsubsection{Baumartensperifische Betrachtung der Nutzungen}

Da die Vorratsstruktur (Durchmesser/Alter) innerhalb und zwischen den Baumartengruppen in Niedersachsen teilweise deutliche Unterschiede aufweist und sich somit unterschiedliche Nutzungs- und Verwendungsoptionen ergeben, müssen die Nutzungspotenziale baumartenspezifisch aufbereitet werden. Im Folgenden sollen die Nutzungsmassen der vier Hauptwirtschaftsbaumarten Niedersachsens Buche, Fichte, Kiefer und Eiche betrachtet werden.

Der Nutzungsanteil der Eiche am Gesamteinschlag liegt im Mittel über alle Varianten zu Simulationsbeginn bei ca. 11 Prozent und geht auf ca. 6 Prozent zurück. Entgegen dem generellen Trend der ansteigenden Endnutzung ist somit bei allen Szenarien ein Nutzungsrückgang bei Eiche insgesamt erkennbar. Werden in der ersten Simulationsperiode jährlich noch 0,4 („,naturschutzorientiert") bis 0,69 (,ertragsorientiert“) Mio. Efm Eiche endgenutzt, ist in der letzten Periode nur noch mit Massen zwischen 0,1 und 0,3 Mio. Efm zu rechnen. Hinsichtlich der Vornutzung tritt ein Rückgang von ca. 0,55 Mio. Efm auf ca. 0,23 Mio. Efm auf. Der mittlere jährliche Gesamteinschlag in den Jahren zwischen 2011 und 2036 beträgt für den „naturnahen Waldbau“ 0,65 Mio. Efm, für die „ertragsorientierte“ Variante 0,77 Mio. Efm und für die „,naturschutzorientierte“ Variante 0,54 Mio. Efm (vgl. Abb. 39). 


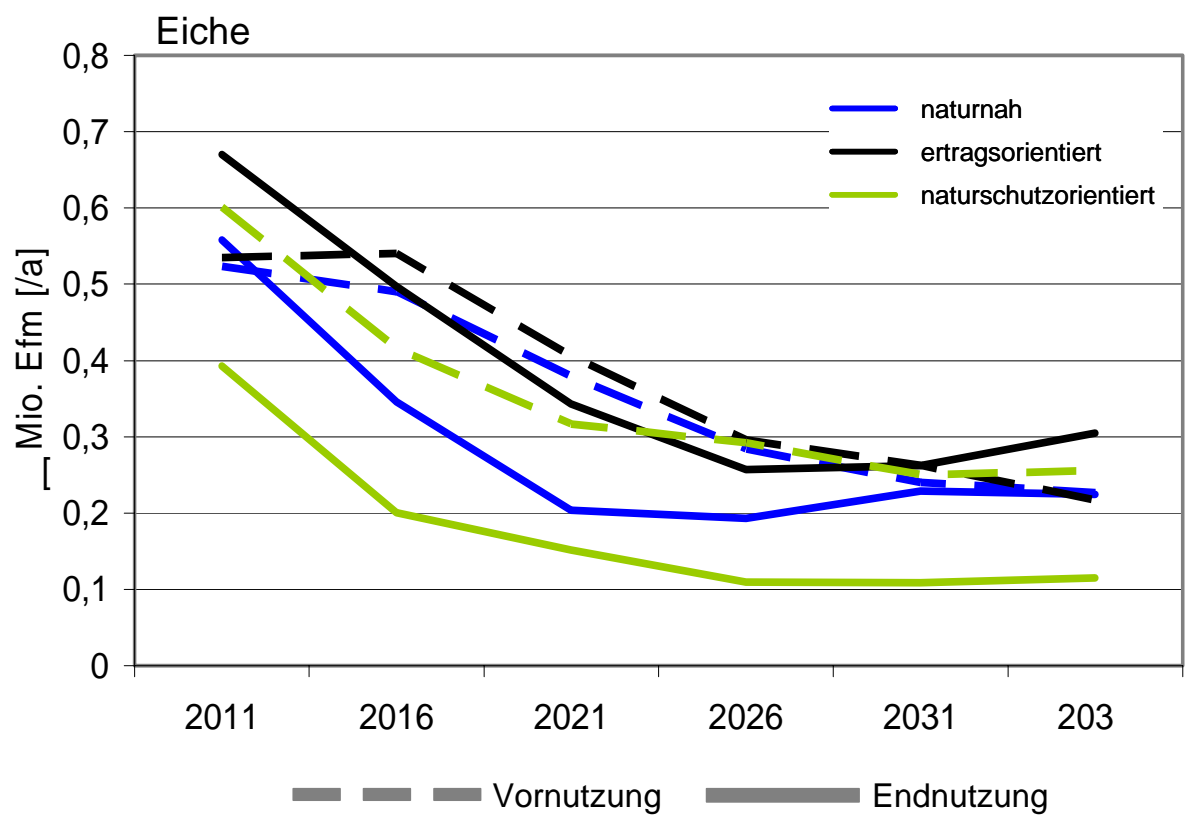

Abbildung 39: Absolute Nutzungsmassen bei der Baumartengruppe Eiche (Efm/a)

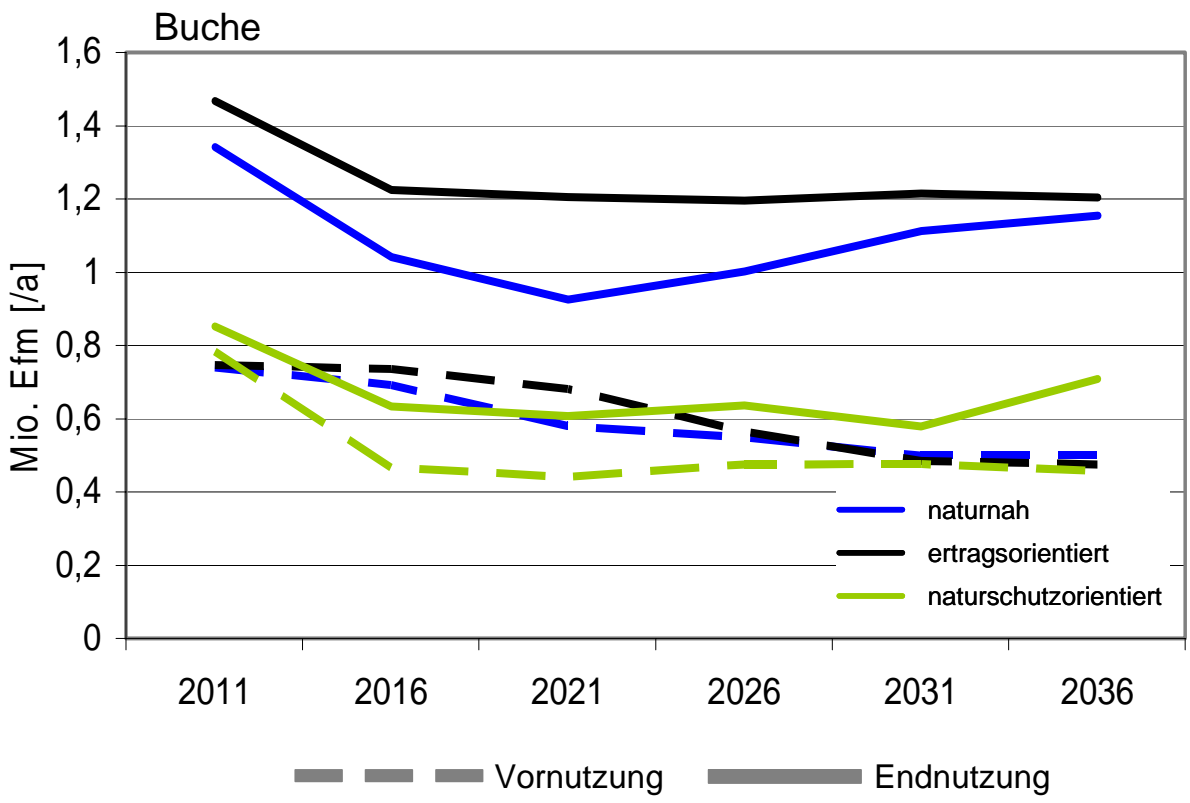

Abbildung 40: Absolute Nutzungsmassen bei der Baumartengruppe Buche (Efm/a) 
Die Nutzungsmassen der zweiten Laubbaumart Buche sind innerhalb der Simulationsperiode homogener verteilt. Durchschnittlich beträgt der Anteil am niedersächsischen Gesamteinschlag ca. 20 Prozent. Dies entspricht beim „,naturnahen Waldbau“ einem mittleren Gesamtbuchenholzeinschlag von 1,69 Mio. Efm/a („ertragsorientiert“ 1,87 Mio. Efm/a, „naturschutzorientiert“ 1,18 Mio. Efm/a). Unter den Annahmen der ,ertragsorientierten“ Variante fallen in der ersten Simulationsperiode über 1,4 Mio. Efm/a Buchenholz aus der Endnutzung an. Dieser Wert geht in der zweiten Periode auf ca. 1,2 Mio. Efm/a zurück und hält dieses Niveau bis zum Simulationsende. Die jährliche Vornutzungsmasse liegt bei einem leicht fallenden Trend zwischen ca. 0,75 und 0,5 Mio. Efm. Vergleichbar, in der zweiten und dritten Simulationsperiode etwas geringer, gestaltet sich die Vornutzung im „naturnahen Waldbau“. Die Endnutzung hingegen fällt insgesamt geringer aus und sinkt bis zum Jahr 2021 unter 1 Mio. Efm/a, um dann wieder bis auf knapp unter 1,2 Mio. Efm/a anzusteigen. Unter den Annahmen eines ,naturschutzorientierten Waldbaus" liegt die jährliche Endnutzungsmasse bei ca. 0.6 Mio. Efm. Eine Ausnahme stellt die erste und letzte Simulationsperiode da. Hier ist die Endnutzung um ca. 0.2 bzw. 0.1 Mio. Efm höher (vgl. Abb. 40).

Der größte absolute Holzeinschlag ist bei der Baumart Fichte zu verzeichnen. Auch bei dieser Baumart ist hinsichtlich der Vornutzungen ein rückläufiger Trend zu beobachten. Die Endnutzungsmassen steigen in allen drei Varianten ab 2016 an. Nur bei der „ertragsorientierten“ Variante fällt die Endnutzungsmasse (ca. 2 Mio. Efm) in der letzten Periode auf das Niveau der ,naturnahen“ Variante ab. Die Spanne des im Gesamtsimulationszeitraum mittleren jährlichen Fichteneinschlags reicht von 1,60 Mio. Efm („,naturschutzorientiert“) bis 2,68 Mio Efm („ertragsorientiert“). Bei 2,54 Mio. Efm/a Fichtenholz liegt das durchschnittliche Nutzungspotenzial in der Variante ,naturnaher Waldbau“ (vgl. Abb. 41).

Bei der Kiefer fallen im Simulationszeitraum durchschnittlich 1,24 Mio. Efm/a bei der Variante ,naturnaher Waldbau“ an. Die Erträge aus Vor- und Endnutzung liegen bei der Kiefer unter den Vorraussetzungen des „,naturnahen Waldbaus“ bis zum Jahr 2031 eng beieinander. Danach ist ein gegenläufiger Trend erkennbar. Die „ertragsorientierte“ Variante führt zu einem mittleren jährlichen Gesamteinschlag von 1,71 Mio. Efm, wobei die Erträge aus Vornutzungen im Vergleich zu den anderen Varianten am stärksten zurückgehen. Das geringste Potenzial weist der „,naturschutzorientierte Waldbau“ auf. Hier fallen jährlich durchschnittlich 0,85 Mio. Efm Kiefernholz an. Bei dieser Variante liegen die Vornutzungsmassen kontinuierlich über den Endnutzungsmassen (vgl. Abb. 42). 


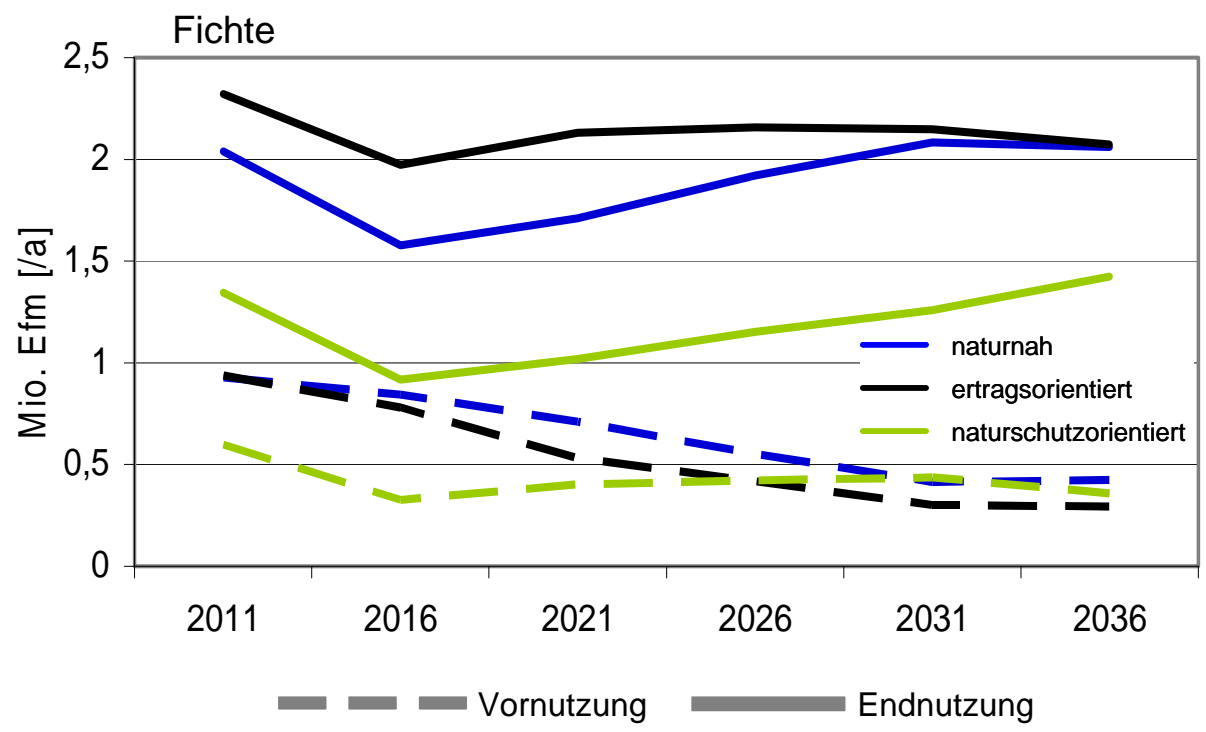

Abbildung 41: Absolute Nutzungsmassen bei der Baumartengruppe Fichte (Efm/a)

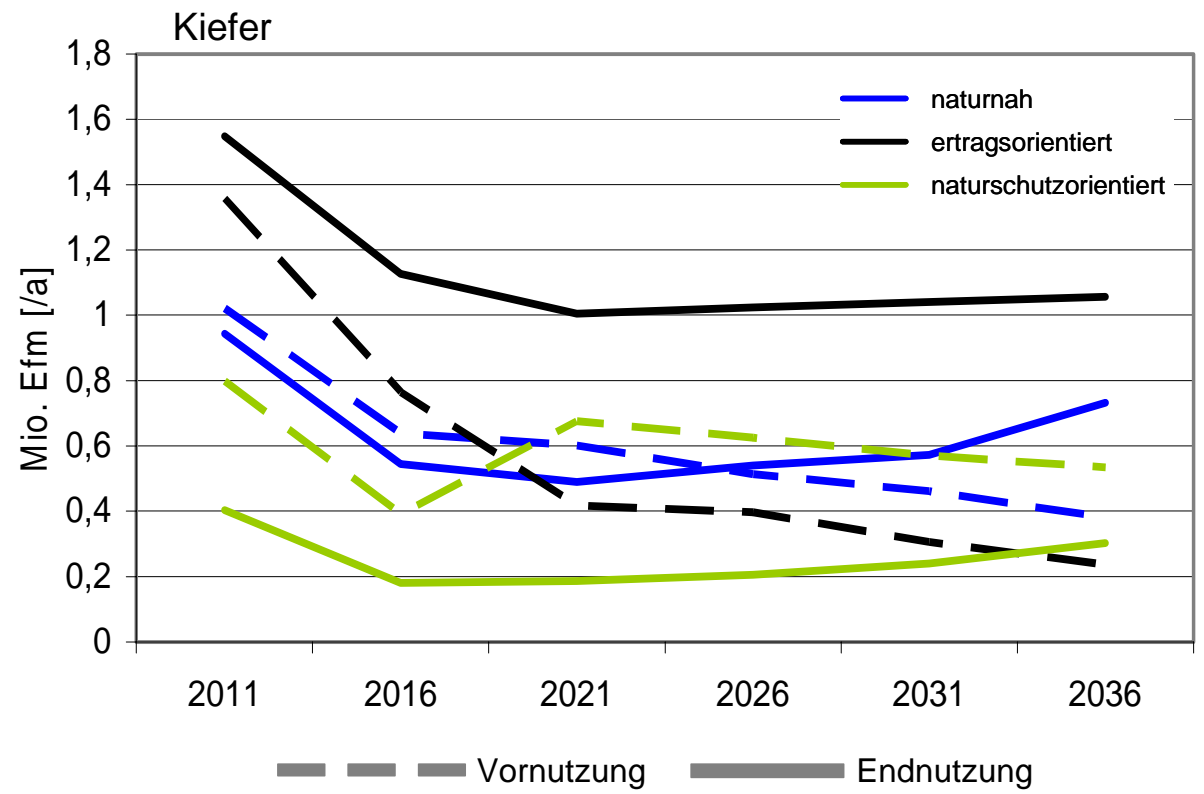

Abbildung 42: Absolute Nutzungsmassen bei der Baumartengruppe Kiefer (Efm/a) 


\subsubsection{Sortenertrag}

Hinsichtlich der weiteren Holzverwertung ist die Differenzierung der Holzpotenziale nach Sortimenten eine wichtige Informationsquelle. Die zugrunde liegenden Modellrechnungen gehen von zwei Leitsortimenten (Aushaltung lang und in Abschnitten) aus, welche nach Stärkeklassen weiter untergliedert werden. Im Folgenden werden die Ergebnisse der Sortierung auf Basis der drei Szenarien dargestellt. Die Tabellen 12 bis 15 zeigen für die Hauptwirtschaftsbaumarten Niedersachsens prognostizierte Sortenerträge getrennt nach Mittenstärkeklassen. Für jedes Szenario werden drei Zeitpunkte (2011, 2026 und 2036) berücksichtigt, wobei die absoluten jährlichen, über den jeweiligen fünfjährigen Prognosezeitraum gemittelten, Erträge dargestellt sind.

Bei der Buche ist im Szenario „,naturnaher Waldbau“ bei allen Stärkeklassen mit Ausnahme der Klassen 4a, 4b und 5a ein Rückgang der Erträge innerhalb von 25 Jahren erkennbar. Im Szenario „,naturschutzorientierter Waldbau“ verschiebt sich dieses Muster um eine Stärkeklasse nach oben, im ,ertragsorientierten Waldbau“" sind lediglich für die Stärkeklassen 4a und 4b vergleichsweise zum Jahr 2011 höhere Massen potenziell realisierbar (vgl. Tab. 12).

Tabelle 12: Buchenholznutzung nach Sortimenten für die Szenarien „naturnaher Waldbau“ (nn), „naturschutzorientierter Waldbau" (no) und „ertragsorientierter Waldbau" (eo) in den Jahren 2011, 2016, 2036

Buche, lang und Abschnitte, 1000 Efm o. R./a

\begin{tabular}{|c|c|c|c|c|c|c|c|c|c|c|c|c|}
\hline & & $1 \mathrm{a}$ & $1 \mathrm{~b}$ & $2 \mathrm{a}$ & $2 \mathrm{~b}$ & $3 a$ & $3 \mathrm{~b}$ & $4 a$ & $4 \mathrm{~b}$ & $5 a$ & $5 \mathrm{~b}$ & $\geq 6$ \\
\hline \multirow{3}{*}{$\Xi$} & 2011 & 46.6 & 110.7 & 64.1 & 141.5 & 124.9 & 84.8 & 44.0 & 131.2 & 236.8 & 200.5 & 473.7 \\
\hline & 2026 & 19.3 & 69.2 & 66.9 & 84.0 & 76.1 & 73.7 & 56.2 & 315.7 & 323.5 & 70.4 & 84.5 \\
\hline & 2036 & 17.1 & 48.2 & 37.4 & 76.9 & 78.3 & 77.7 & 54.1 & 402.9 & 390.6 & 61.2 & 52.3 \\
\hline \multirow{3}{*}{$\stackrel{8}{g}$} & 2011 & 42.1 & & 73.0 & & & 74.3 & & & & & 357.9 \\
\hline & 2026 & 13.6 & 60.1 & 53.9 & 69.7 & 57.4 & 48.7 & 51.1 & 29.3 & 302.0 & 140.1 & 58.7 \\
\hline & 2036 & 7.8 & 31.4 & 38.9 & 64.0 & 71.2 & 66.3 & 45.5 & 37.2 & 331.8 & 183.2 & 36.9 \\
\hline \multirow{3}{*}{ \& } & 2011 & 40.7 & 132.7 & 74.2 & 181.5 & 127.4 & 72.4 & 60.6 & 256.4 & 229.7 & 218.4 & 472.6 \\
\hline & 2026 & 20.0 & 86.0 & 71.0 & 93.1 & 97.8 & 81.8 & 195.0 & 511.5 & 173.3 & 79.4 & 90.1 \\
\hline & 2036 & 21.2 & 52.8 & 46.1 & 83.0 & 88.8 & 65.1 & 203.1 & 662.9 & 113.2 & 59.8 & 45.5 \\
\hline
\end{tabular}

Bei der Eiche ergibt sich ein ähnliches Bild. Der deutliche Rückgang der starken Laubholzsortimente $(\geq 5 \mathrm{~b})$ resultiert aus einer konsequenten Zielstärkennutzung der dicksten Bäume eines Bestands (vgl. Tab. 13). 
Tabelle 13: Eichenholznutzung nach Sortimenten für die Szenarien "naturnaber Waldbau" (nn), „naturschutzorientierter Waldbau" (no) und „ertragsorientierter Waldbau" (eo) in den Jabren 2011, 2016, 2036

Eiche, lang und Abschnitte, 1000 Efm o. R./a

\begin{tabular}{|c|c|c|c|c|c|c|c|c|c|c|c|c|}
\hline & & $1 \mathrm{a}$ & $1 \mathrm{~b}$ & $2 a$ & $2 \mathrm{~b}$ & $3 a$ & $3 b$ & $4 a$ & $4 \mathrm{~b}$ & $5 a$ & $5 b$ & $\geq 6$ \\
\hline \multirow{3}{*}{$\Xi$} & 2011 & 35.6 & 53.7 & 63.3 & 70.0 & 78.2 & 42.8 & 26.1 & 14.9 & 46.0 & 112.7 & 253.8 \\
\hline & 2026 & 19.3 & 33.7 & 33.3 & 37.6 & 33.4 & 24.4 & 19.1 & 12.8 & 92.7 & 41.6 & 15.5 \\
\hline & 2036 & 9.7 & 21.5 & 32.2 & 33.2 & 26.3 & 30.6 & 8.7 & 15.7 & 124.0 & 46.1 & 0.0 \\
\hline \multirow{3}{*}{ ఏ } & 2011 & 54.5 & 67.1 & 66.5 & 73.6 & 58.7 & 44.1 & 29.6 & 27.4 & 16.7 & 77.0 & 212.9 \\
\hline & 2026 & 14.1 & 23.9 & 27.8 & 33.6 & 30.3 & 26.7 & 26.3 & 20.2 & 16.6 & 61.0 & 24.1 \\
\hline & 2036 & 8.0 & 17.9 & 27.1 & 30.6 & 28.7 & 21.4 & 25.9 & 21.0 & 7.6 & 79.3 & 17.6 \\
\hline \multirow{3}{*}{ \& } & 2011 & 36.6 & 63.5 & 72.1 & 83.6 & 78.6 & 43.9 & 23.5 & 26.7 & 123.7 & 124.6 & 246.5 \\
\hline & 2026 & 19.5 & 39.1 & 41.2 & 37.3 & 36.4 & 30.1 & 15.9 & 88.2 & 89.6 & 19.1 & 15.8 \\
\hline & 2036 & 14.7 & 27.0 & 27.1 & 29.9 & 27.0 & 20.9 & 16.1 & 132.7 & 94.6 & 7.0 & 0.0 \\
\hline
\end{tabular}

Bei Fichte sind die im Jahr 2011 anfallenden Massen bei den Abschnitten in den Stärkeklassen von $1 \mathrm{~b}$ bis $4 \mathrm{a}$ in allen Szenarien annähernd gleich verteilt. Es fallen je Stärkeklasse bei einem „naturnahen Waldbau“ um die 0,3 Mio. Efm/a an (,naturschutzorientiert“ knapp 0,2 Mio. Efm/a, ,ertragsorientiert“ ca. 0,4 Mio. Efm/a). Über die Stärkeklasse 4a hinaus fallen die Massen mit zunehmendem Mittendurchmesser ab. Die zeitliche Entwicklung der Verteilung der Abschnitte auf die einzelnen Stärkeklassen zeigt einen deutlichen Rückgang bei den schwächeren Sortimenten und eine Zunahme bei stärkeren Dimensionen (vgl. Tab. 14). Eine Ursache hierfür liegt in der Altersklassenausstattung der Fichte zum Startzeitpunkt der Simulation (vgl. Abb. 2).

Tabelle 14: Fichtenbolznutzung nach Sortimenten fïr die Szenarien "naturnaber Waldbau" (nn), „naturschutzorientierter Waldbau" (no) und „ertragsorientierter Waldbau" (eo) in den Jabren 2011, 2016, 2036

Fichtenabschnitte, $1000 \mathrm{Efm}$ o. R./a

\begin{tabular}{|c|c|c|c|c|c|c|c|c|c|c|c|c|}
\hline & & $1 \mathrm{a}$ & $1 \mathrm{~b}$ & $2 \mathrm{a}$ & $2 \mathrm{~b}$ & $3 a$ & $3 \mathrm{~b}$ & $4 a$ & $4 \mathrm{~b}$ & $5 \mathrm{a}$ & $5 b$ & $\geq 6$ \\
\hline \multirow{3}{*}{$\Xi$} & 2011 & 124.1 & 655 & 60.8 & 13.8 & 27.6 & 356.1 & 304.8 & 180.2 & 93.2 & 47.2 & 39.8 \\
\hline & 2026 & 53.8 & 231.6 & 315.8 & 405.1 & 508.9 & 531.7 & 276.3 & 73.7 & 24.8 & 2.7 & 0.0 \\
\hline & 2036 & 52.5 & 190.1 & 273.2 & 409.0 & 575.8 & 549.4 & 342.2 & 51.4 & 15.3 & 3.9 & 2.0 \\
\hline \multirow{3}{*}{ \& } & 2011 & 7.6 & 209 & 199.6 & 171.6 & 177.2 & 179.6 & 206.0 & 155.0 & 79.3 & 42.3 & 38.7 \\
\hline & 2026 & 30.2 & 129.8 & 180.0 & 219.0 & 266.2 & 252 & & 96.8 & 23.1 & & 0.0 \\
\hline & 2036 & 27.4 & 122.3 & 174.9 & 234.8 & 319.0 & 338.7 & 404.3 & 109.3 & 21.3 & 5.1 & 0.6 \\
\hline \multirow{3}{*}{8} & 2011 & 151.6 & 4 & 43 & 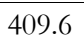 & 427.4 & 4 & & 1 & 94 & 47.1 & 40.0 \\
\hline & 2026 & 62.4 & 263.9 & 376.6 & 559.3 & 605.0 & 574.0 & 15 & 76.9 & 25.6 & 3.4 & 0.0 \\
\hline & 2036 & 57.9 & 211.4 & 329.1 & 534.5 & 612.3 & 561.5 & 122.6 & 49.9 & 14.5 & 3.7 & 1.2 \\
\hline
\end{tabular}


Noch deutlicher zeigt sich diese Entwicklung bei der Kiefer (vgl. Tab. 15). Bei den Abschnitten entfallen im Jahr 2011 die größten Massen auf die Stärkeklassen 1b und 2a („naturnaher Waldbau“ ca. 0,35 Mio. Efm/a bzw. ca. 0,34 Mio. Efm/a). Bereits 5 Jahre später ist in diesen Stärkelassen ein erheblicher Rückgang der Potenziale erkennbar, welcher bis 2036 andauert.

Tabelle 15: Kiefernholz̧nutzung nach Sortimenten für die Szenarien „naturnaher Waldbau“ (nn), „,naturschutzorientierter Waldbau" (no) und ,ertragsorientierter Waldbau" (eo) in den Jabren 2011, 2016, 2036

Kiefernabschnitte, 1000 Efm o. R./a

\begin{tabular}{rr|r|r|r|r|r|r|r|r|r|r|r} 
& & $1 \mathrm{a}$ & $1 \mathrm{~b}$ & $2 \mathrm{a}$ & $2 \mathrm{~b}$ & $3 \mathrm{a}$ & $3 \mathrm{~b}$ & $4 \mathrm{a}$ & $4 \mathrm{~b}$ & $5 \mathrm{a}$ & $5 \mathrm{~b}$ & $\geq 6$ \\
\hline \multirow{2}{*}{2011} & 132.9 & 345.1 & 335.3 & 259.1 & 228.7 & 253.8 & 177.4 & 59.8 & 18.6 & 2.3 & 1.5 \\
2026 & 35.9 & 146.4 & 204.0 & 220.1 & 192.8 & 207.8 & 16.0 & 0.7 & 0.0 & 0.0 & 0.0 \\
& 2036 & 23.6 & 110.9 & 185.2 & 241.7 & 243.5 & 274.9 & 16.3 & 0.3 & 0.0 & 0.0 & 0.0 \\
\hline \multirow{2}{*}{2011} & 109.5 & 248.8 & 209.5 & 133.8 & 94.4 & 99.6 & 104.1 & 49.9 & 13.2 & 1.8 & 1.6 \\
& 2026 & 38.5 & 138.8 & 173.4 & 159.2 & 122.9 & 83.1 & 79.4 & 2.5 & 0.5 & 0.0 & 0.0 \\
& 2036 & 21.7 & 90.4 & 143.8 & 172.8 & 153.3 & 119.5 & 117.3 & 3.8 & 0.7 & 0.0 & 0.0 \\
\hline \multirow{2}{*}{2011} & 158.0 & 534.3 & 565.1 & 450.5 & 411.7 & 406.1 & 180.2 & 59.4 & 18.3 & 2.3 & 1.6 \\
\multirow{2}{*}{2026} & 47.5 & 198.5 & 264.9 & 392.6 & 385.9 & 109.5 & 8.7 & 0.4 & 0.0 & 0.0 & 0.0 \\
& 2036 & 28.1 & 143.6 & 229.5 & 417.6 & 415.5 & 75.8 & 4.3 & 0.7 & 0.5 & 0.0 & 0.0 \\
\hline
\end{tabular}

\subsubsection{Berücksichtigung eigentümerspezifischer und naturaler Rabmenbedingungen}

Die zuvor vorgestellten Erträge basieren auf der Annahme eines uneingeschränkten Abschöpfens der szenariospezifischen Potenziale. Diese werden unter Berücksichtigung eines eigentümerspezifischen Nutzungsverhaltens jedoch reduziert. Im Folgenden werden die auf Basis des zwischen 1987 und 2002 ermittelten Nutzungsmusters modellierten Potenzialeinschränkungen für die ersten fünfzehn Jahre der jeweiligen Szenarien dargestellt.

Für Niedersachsen ergibt sich insgesamt bei allen Szenarien eine gleich starke Reduktion der Potenziale. Beim „,naturnahen“ und „,naturschutzorientierten Waldbau" ist mit einer Reduktion von $31 \%$ der Nutzungspotenziale zu rechnen. Bei der „Ertragsvariante“ beträgt der Nutzenentgang $32 \%$.

Differenziert man die Nutzungsreduktion nach Eigentumsart, zeigt sich, dass im Privatwald die Potenziale am stärksten (38\%) und im Landeswald am geringsten $(23 \%)$ gemindert werden. Auf Flächen des Bundes- bzw. Körperschaftswaldes wird das Potenzial zu ca. $70 \%$ genutzt. Somit entspricht hier die Reduktion dem niedersächsischen Durchschnitt.

Hinsichtlich der Hauptwirtschaftsbaumarten ist bei Eiche die stärkste Potenzialreduktion festzustellen. Basierend auf dem Szenario „,naturnaher Waldbau“ ist im Landeswald das Eichenpotenzial um ca. 30\%, im Privatwald um ca. $47 \%$ herabzusetzen. Insgesamt werden ca. $38 \%$ des Eichenpotenzials nicht genutzt. Bei 
Buche beträgt die Reduktion ca. $24 \%$, bei Fichte $23 \%$ und bei Kiefer $33 \%$ (vgl. Tab. 16).

Tabelle 16: $\quad$ Prozentuale Reduktion der Nutzungspotenziale für die Hauptwirtschaftsbaumarten

\begin{tabular}{l|c|c|c}
\hline & $\begin{array}{c}\text { „naturnah“ } \\
{[\%]}\end{array}$ & $\begin{array}{c}\text { „ertragsorientiert“ } \\
\text { [\%] }\end{array}$ & $\begin{array}{c}\text { „naturschutz- } \\
\text { orientiert“ [\%] }^{\text {[\%] }}\end{array}$ \\
\hline Eiche & 38,1 & 39,4 & 38,5 \\
Buche & 24,4 & 24,8 & 24,6 \\
Fichte & 22,7 & 23,7 & 21,1 \\
Kiefer & 32,7 & 33,2 & 33,6 \\
\hline
\end{tabular}

Diese Betrachtung berücksichtigt jedoch nicht, dass gerade im Landeswald Naturschutzauflagen die Nutzungsmöglichkeiten einschränken bzw. ausschließen. Insbesondere sind bereits heute große Bereiche des niedersächsischen Teils des Nationalparks Harz aus der Nutzung genommen und in den ausgewiesenen 106 Naturwäldern findet auf ca. 4.500 ha keine Nutzung mehr statt. Diese kumulierten Nutzungsverzichte betragen im Simulationszeitraum zwischen 2,4 und 4,1 Mio Vfm (vgl. Tab. 17).

Tabelle 17: Kumulierter Nutzungsverzicht für den simulierten Zeitraum bis 2036 auf von der Nutzung ausgeschlossenen Flächen

\begin{tabular}{c|c|c}
\hline „naturnah“ & „ertragsorientiert“ & „naturschutzorientiert“ \\
\hline 3,6 Mio. Vfm & 4,1 Mio. Vfm & 2,4 Mio. Vfm \\
\hline
\end{tabular}

In den beiden Szenarien „naturnaher“ und „naturschutzorientierter“ Waldbau werden 3 bzw. 10 Habitatbäume pro Hektar angestrebt. Der Vorrat der Habitatbäume beläuft sich am Ende des Simulationszeitraumes auf ca. 2,5 bzw. 44,6 Mio fm. Dies sind ca. 1 \% bzw. $12 \%$ des stehenden Vorrates.

Der Totholzvorrat steigt beim Szenario „ertragsorientierter Waldbau“ im Vergleich zum Ausgangswert 2006 von 8,7 auf 9,9 Mio. Vfm leicht an (vgl. Tab. 18). Die beiden anderen Szenarien, in denen gezielt das Totholz angereichert wurde, führen zu Totholzvorräten von 23,6 Mio Vfm bzw. 34,7 Mio Vfm.

Tabelle 18: Totholzvorrat (Vergleich der Jahre 2006 und 2036)

\begin{tabular}{|c|c|c|c|}
\hline BWI 22006 & „naturnah“ & „ertragsorientiert““ & $\begin{array}{c}\text { „naturschutz- } \\
\text { orientiert“ }\end{array}$ \\
\hline 8,7 Mio. Vfm & 23,6 Mio. Vfm & 9,9 Mio. Vfm & 34,7 Mio. Vfm \\
\hline
\end{tabular}


Insgesamt beläuft sich der durch Naturschutzauflagen bedingte Nutzungsentgang (Nutzungsverzicht + Habitatbaumvorrat + Totholzvorrat) bis zum Jahr 2036 bei der Variante „naturnaher Waldbau“ auf ca. 29,7 Millionen Vfm, bei der „ertragsorientierten“ Variante auf 14,0 Millionen Vfm und bei der „naturschutzorientierten" Variante auf 81,7 Millionen Vfm. 



\section{Schlussfolgerungen}

Die volkswirtschaftliche Abgrenzung eines Clusters Forst und Holz ist stets mehr oder weniger willkürlich, da der Rohstoff Holz im Verarbeitungsprozess immer stärker in anderen Produkten aufgeht und die wirtschaftliche Bedeutung des Holzanteils damit immer weiter abnimmt. Trotzdem ist eine funktionale Clusterdefinition sinnvoll, da der Rohstoff Holz und die daraus hervorgehenden Halbwaren eine oft nicht zu substituierende Grundlage für die entsprechenden Endprodukte sind. Die für das vorliegende Projekt gewählte Abgrenzung ist an der Definition des Europäischen Parlamentes orientiert und umfasst die Wirtschaftszweige Forstwirtschaft, Holzbe- und Holzverarbeitung, Papiergewerbe, Verlagsund Druckereigewerbe, Baugewerbe mit Holz sowie Handelsvermittlung und Großhandel mit Holz.

Neben dieser Abgrenzung in der Verarbeitungsrichtung ist auch eine räumliche Abgrenzung schwierig. Die Grenzen des Clusters Forst und Holz Niedersachsen sind naturgemäß nicht leicht zu definieren, da viele wirtschaftliche Verflechtungen $\mathrm{zu}$ anderen Bundesländern bestehen, die, anders als z. B. der Außenhandel über die Bundesgrenzen hinweg, statistisch nicht erfasst werden. Trotz dieser Schwierigkeit wurde mit diesem Projekt versucht, die Bedeutung des Clusters Forst und Holz in Niedersachsen und dessen Entwicklungsmöglichkeit zu untersuchen.

Der Cluster Forst und Holz ist auch in Niedersachen ein ähnlich wichtiger Bestandteil der Gesamtwirtschaft wie in der Bundesrepublik Deutschland insgesamt. Der Anteil des Clusters am Umsatz beträgt 3,4 \%, der Anteil an der Bruttowertschöpfung gut $2 \%$. Gemessen am Umsatz besitzt das Verlags- und Druckereigewerbe mit $30 \%$ den höchsten Anteil innerhalb des Clusters. Im Jahr 2006 waren knapp 80.000 Menschen im Cluster Forst und Holz Niedersachsen beschäftigt. Auch in Hinblick auf die Beschäftigtenzahlen ist das Verlags- und Druckereigewerbe innerhalb des Clusters führend.

Will man die Entwicklungsmöglichkeiten des Clusters Forst und Holz einschätzen, rückt aufgrund der Abhängigkeit des Clusters vom Rohstoff Holz die Frage nach dem noch ungenutzten Rohholzpotenzialen in den Vordergrund. Daher wurden im vorliegenden Projekt auf der Grundlage der zweiten Bundeswaldinventur die Rohholzpotenziale für drei verschiedene Bewirtschaftungsszenarien abgeschätzt: (1) „ertragsorientierte“, (2) „naturnahe“ und (3) „,naturschutzorientierte" Waldbewirtschaftung. Die Ergebnisse zeigen, dass vergleichsweise moderate Änderungen in der Durchforstungsintensität und der Zielstärke bei konsequenter Umsetzung einen deutlichen Einfluss auf die Vorratshaltung und die Nutzungen haben. Im Vergleich zur Ausgangssituation führt die Variante „naturnaher Waldbau“ zu einer leichten Vorratsabsenkung, die „Ertragsvariante“ zu einem deutlichen Vorratsabbau und die „Naturschutzvariante“ zu einem ebenso starken Vorratsaufbau. In den nächsten 30 Jahren nimmt in Niedersachsen der 
Anteil der Vornutzungen an der Gesamtnutzung stark ab. Dies erklärt sich durch den Altersklassenaufbau und den in der Vergangenheit zurückgefahrenen Nadelholzanbau. Die Zuwächse werden aus den gleichen Gründen stagnieren oder leicht fallen, während die Nutzungen zunächst steigen werden, um sich dann auf einem nicht ganz so hohen Niveau einzupendeln. Erhöhte Naturschutzauflagen können zu einem erheblichen Nutzungsverzicht führen.

Vergleicht man die aus den Szenarien resultierenden Rohholznutzungspotenziale mit den aktuellen Einschlägen aus der amtlichen Holzeinschlagsstatistik (zum Problem der Unterschätzung des Einschlages in der amtlichen Statistik siehe DiETER u. ENGLERT 2005), ergibt sich vor allem für die Periode 2008 bis 2011 noch ein einheitlich positives Bild (vgl. Abb. 43). Es besteht ein deutlicher Potenzialüberschuss, der sich aus der Einbeziehung aller Stichprobenpunkte der BWI 2 in die Bewirtschaftung nach gleichen Regeln ergibt. Für die Jahre danach sinken die Nutzungsmöglichkeiten allerdings für alle drei Szenarien ab und sie liegen beim „,naturschutzorientierten“ Szenario ab 2012 zeitweise sogar unter dem Niveau des derzeitigen Holzeinschlages.

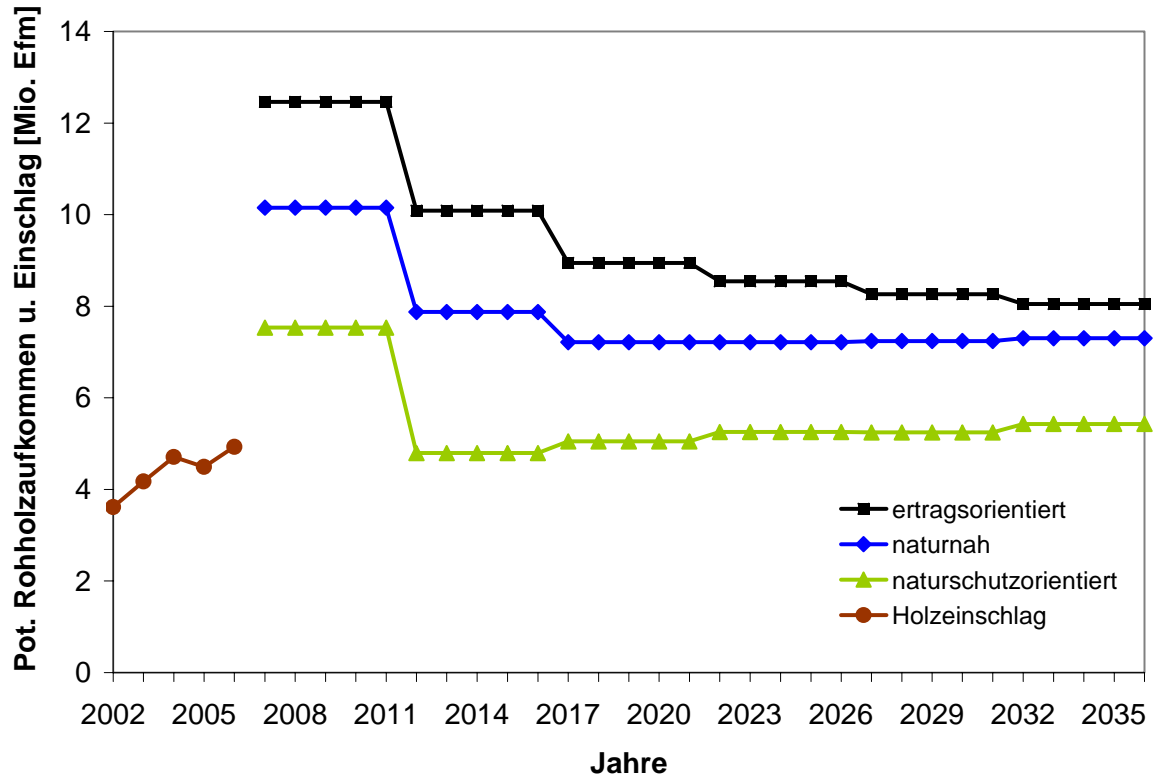

Abbildung 43: Einschlag und potenzielles jährliches Robholzaufkommen in Niedersachsen, letzteres für die drei verschiedenen Szenarien (,ertragsorientiert", „naturnab" und ,naturschutzorientiert")

Die Gegenüberstellung von aktuellem Holzeinschlag und potenziellem Rohholzaufkommen für die vier Holzartengruppen zeigt ein differenzierteres Bild. Während sich beim Laubholz für alle drei Szenarien ein ungenutztes Potenzial abzeichnet, scheint dies beim Nadelholz nicht der Fall zu sein. Im Vergleich mit 
dem Holzeinschlag 2006 weisen die Modellrechnungen bei der Holzartengruppe Kiefer nur für die „ertragsorientierte Waldbewirtschaftung“ einen Potenzialüberschuss aus. Bei „naturnaher“ oder „,naturschutzorientierter“ Bewirtschaftung liegt das modellierte Potenzial unter dem aktuellen Einschlag. Für die Holzartengruppe Fichte gilt dies - zumindest in geringem Umfang - auch für das „,naturschutzorientierte“ Bewirtschaftungsszenario (s. Abb. 44). Die Verlagerung der Nutzungsschwerpunkte von der Vor- zur Endnutzung ist vor dem Hintergrund der daran gekoppelten Wertschöpfungsketten als dramatische Veränderungen zu bezeichnen. Dies gilt besonders für die Baumartengruppen Eiche, Fichte und Kiefer (vgl. Abb. 38 - 42). Es zeichnen sich demzufolge Versorgungsengpässe für die Papier- und Holzwerkstoffindustrie ab, die in den letzten Jahrzehnten einen enormen Aufschwung zu verzeichnen hatten.

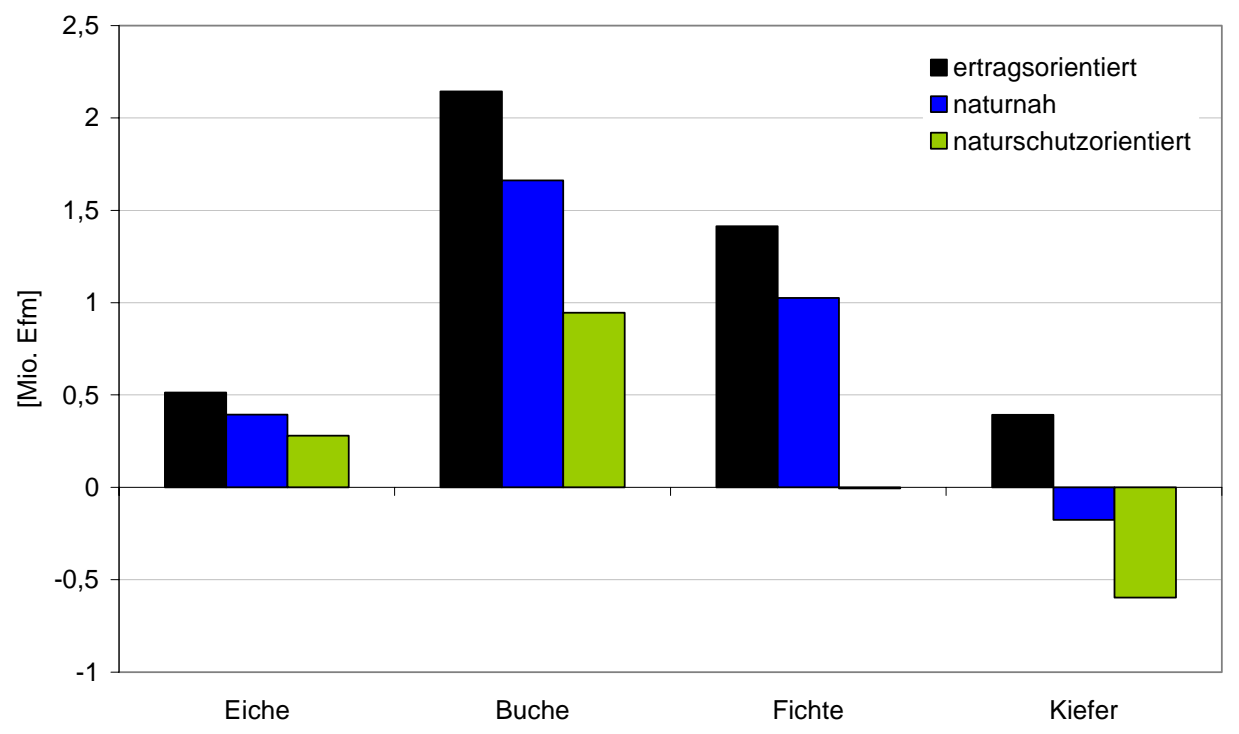

Abbildung 44: Differenz zwischen dem potenziellen jährlichen Robholzaufkommen (Durchschnitt 20072036) und dem Holzeinschlag 2006, nach Holzartengruppen und Szenarien

Aus den Daten der beiden Bundeswaldinventuren 1987 und 2002 lassen sich die Nutzungen innerhalb dieses Zeitraumes rekonstruieren. Für das vorliegende Projekt wurden diese Nutzungen daraufhin analysiert, in welchen Beständen, nach Alter, Besitzart, Lage etc., eine Nutzung ganz unterlassen wurde. Die gefundenen statistischen Zusammenhänge wurden dann auf das potenzielle Rohholzaufkommen der drei Szenarien übertragen, indem die Stichprobenpunkte entsprechend ihrer individuellen Nichtnutzungswahrscheinlichkeit aus dem Potenzial ausgeschlossen wurden. Die prozentualen Reduktionen variieren nicht sehr stark zwischen den Szenarien, dafür aber stärker bei den Besitzarten und den Holzartengruppen. Insbesondere der Kleinprivatwald $<20$ ha, in dem ca. $25 \%$ des 
landesweiten Gesamtvorrates stocken, hat ein Mobilisierungsproblem (vgl. Abb. 30). Bei den Baumartengruppen reduziert sich am stärksten das potenzielle Rohholzaufkommen der Eiche (durchschnittlich - 38,7\%), am schwächsten dasjenige der Fichte (durchschnittlich - 22,5\%).

Wird die gleiche Wahrscheinlichkeit einer Nichtnutzung unterstellt wie in der Vergangenheit und das potenzielle jährliche Rohholzaufkommen jeweils entsprechend reduziert, so fällt das Potenzial deutlich niedriger aus. Für die Holzartengruppe Kiefer liegt das reduzierte durchschnittliche jährliche Rohholzpotenzial dann für alle Szenarien unterhalb des aktuellen Einschlages. Selbst in der ersten Periode ist das reduzierte Potenzial niedriger als der Einschlag 2006. Dies weist einerseits darauf hin, dass die oben stehende Annahme nicht voll zutreffend ist und die jüngsten Mobilisierungsaktivitäten, z. B. der Landwirtschaftskammer, sowie die steigenden Holzpreise bereits eine höhere Nutzungsbereitschaft erzeugt haben (nachgewiesen durch den Einschlag oberhalb des reduzierten Potenzials). Andererseits zeigt das Ergebnis aber auch, dass zusätzliche Potenziale nur mobilisiert werden können, wenn auch Waldbesitzer ohne bisheriges Nutzungsinteresse für eine Bewirtschaftung gewonnen werden können. Die Ergebnisse der Szenariorechnungen weisen damit eindrücklich darauf hin, dass der wichtigste Ansatzpunkt für eine vermehrte Holzmobilisierung die künftige Bewirtschaftung von Wald darstellt, der bisher nicht bewirtschaftet wurde. In bereits heute intensiv nutzenden Forstbetrieben mit einem guten Pflegezustand lassen sich hingegen durch eine Modifikation der Waldbaustrategien kaum noch zusätzliche Rohholzmengen mobilisieren, ohne die Nachhaltigkeit zu gefährden (vgl. unterschiedliche Szenarien).

Alternativ zu einer stärkeren Rohholzmobilisierung in Niedersachsen könnte auch die Beschaffung aus benachbarten Bundesländern erwogen werden. Der Bedarf besteht am ehesten für das sich stark verknappende Nadelholz. Hierfür sind die Ergebnisse der im Rahmen des Projektes ebenfalls durchgeführten Holzstromanalyse interessant. Danach gehen ca. 1,1 Mio. $\mathrm{m}^{3}$ sägefähiges Nadelholz an die niedersächsische Nadelholzsägeindustrie. Deren Bedarf liegt aber bei ca. 1,8 Mio. $\mathrm{m}^{3}$. Dies deutet darauf hin, dass etwa ein Drittel des Rohstoffbedarfs dieser Branche von außerhalb Niedersachsens kommt. Eine Ausweitung des Bezuges wäre denkbar, dürfte aber wegen der hohen Verarbeitungskapazitäten in den benachbarten Bundesländern nur begrenzt möglich sein. Wegen des hohen Anteils, den der Holzhandel bei der Vermarktung des Industrieholzes einnimmt, ist zu diesem Sortiment eine ähnliche Analyse nicht möglich.

Angesichts wachsender Produktionskapazitäten in der Holzindustrie und einer weiter steigenden Energieholznachfrage werden sich die bereits heute abzeichnenden Versorgungsengpässe und Verteilungsprobleme am Rohholzmarkt weiter verschärfen. Der Druck der Nachfrageseite auf den Rohholzmarkt darf jedoch nicht dazu führen, dass die Forstwirtschaft die Grundsätze der Nachhaltigkeit verletzt. Rohholzpotenziale lassen sich nur einmal verplanen, was bei der 
Entscheidung für neue Werke oder den Ausbau der bestehenden Holzverarbeitungskapazitäten zu berücksichtigen ist. Die Rohholzmobilisierung ist aber nur ein wichtiger Aspekt der Stärkung des Clusters Forst und Holz. Die Verbesserung der wirtschaftlichen Rahmenbedingungen für die meist mittelständisch geprägten Branchen des Clusters Forst und Holz wäre ein anderer wichtiger Aspekt, der aber mit dem vorliegenden Projekt nicht tiefer bearbeitet werden konnte. 



\section{Ausblick}

Die Autoren der vorliegenden Studie haben sich bemüht, wichtige Daten und Fakten für den Forst und Holz Cluster Niedersachsen zusammenzutragen und anhand von Szenarien mögliche zukünftige Entwicklungen der Rohholzpotenziale für dieses Bundesland aufzuzeigen. Es liegt in der Natur der Sache, dass ein solcher, in die Zukunft gerichteter, Ansatz nicht ohne Probleme zu realisieren ist, denn zukünftige Entwicklungen sind stets ungewiss.

Aber bereits die Information über den aktuellen Zustand der Holzvorräte und Nutzungen in Niedersachsen sind mit Unsicherheiten behaftet. Seit der BWI 2 sind mittlerweile 5 Jahre vergangen; während dieser Zeit gab es starke Zunahmen der Holzeinschläge und geradezu dramatische Entwicklungen auf den Holzmärkten. Ein Übriges hat der Sturm Kyrill im Januar 2007 bewirkt. Zwar wurde versucht, diese jüngsten Entwicklungen bei der Beschreibung der Ausgangssituation mit einzubeziehen, womit aber nicht alle sich daraus ergebenden Unsicherheiten ausgeschlossen sind.

Letztendlich geht es aber nicht primär um die Zahlen der Vergangenheit, sondern um die der Zukunft. Die Daten der Clusterstudie sollen nach dem Prinzip wirken: „Zahlen stellen nur Fragen, Antworten müssen gefunden werden“!

Mit diesem Anspruch möchte die vorliegende Untersuchung dazu beitragen, dass die dem Cluster angehörenden Betriebe der Forst- und Holzwirtschaft und nachgelagerte Bereiche, die Verwaltungen, Forschungseinrichtungen und die Politik sich den aufgeworfenen Fragen stellen und gemeinsam versuchen, Antworten zur Stärkung des Clusters Forst und Holz in Niedersachsen zu finden. Ziel muss es dabei sein, die derzeit sehr günstigen Entwicklungschancen zur Stärkung der nachhaltigen Produktion und Nutzung des Rohstoffes Holz auszunutzen.

Die Diskussionen sollten auf den Ebenen biologische Produktion, technische Produktion - Dienstleister - Holzindustrie und Politik folgende Punkte aufgreifen:

\section{Ebene: Biologische Produktion}

- Überdenken der z. T. auf „Schwachholzvermeidung“ ausgerichteten Bestandesbehandlungsstrategien

- Stärkere Berücksichtigung der langfristigen Marktbedürfnisse bei der Baumarten-/Herkunftswahl, Sicherung eines angemessenen Nadelholzanteils

- Überdenken der Zielstärken und Umtriebszeiten

- Förderung der nachhaltigen Stabilität der Waldbestände unter Berücksichtigung der neuen Herausforderungen durch den Klimawandel 
- Erhöhung der Nutzungsintensität inkl. „Vollbaumnutzung“, wo nachhaltig möglich

- Erschließung bisher wenig genutzter Rohholzpotenziale, z. B. im Bereich des sonstigen Laubholzes mit niedriger Umtriebszeit

\section{Ebene: Technische Produktion, Dienstleister, Holzindustrie}

- Erschließung bisher nicht genutzter Potenziale (insbesondere im Kleinprivatwald) durch Beratung, Betreuung und Entwicklung geeigneter Betreuungs- und Kooperationsmodelle (wie z. B. Waldpacht)

- Verbesserung der Schnittstelle zwischen Forstwirtschaft und Holzwirtschaft, insbesondere in dem Bereich Logistik, und des Datenaustausches

- Verbesserung der gegenseitigen Informationen über Rohstoff-Ressourcen und Verarbeitungs-Kapazitäten und deren Entwicklung

- Entwicklung neuer effizienter Bearbeitungsverfahren für bisher wenig genutzte Ressourcen (anderes Laubholz mit niedriger Lebensdauer, geringerwertiges Laubstarkholz etc.)

\section{Ebene: Politik}

- Klares Bekenntnis der Politik zu einer im umfassenden Sinne nachhaltigen Rohholzproduktion (in diesem Zusammenhang: Überdenken der Flächenstilllegungspolitik der letzten Jahre und des Zurückfahrens der Forstpflanzenzüchtung)

- Förderung neuer Werke nur unter Berücksichtigung der vorhandenen Rohholzpotenziale

- Entwicklung einer forstlichen Förderpolitik, die den neuen Herausforderungen (Rohstoffproduktion, Klimawandel) gerecht wird

- Minderung der Konkurrenzprobleme zwischen stofflicher Verwertung und Bioenergie

- Fortentwicklung des forstlichen Steuersystems zur Schaffung von Anreizen für waldbauliche Investitionen und Waldnutzungen

- Verbesserung der Datenerfassung und Dokumentation über die Entwicklungen im Cluster Forst und Holz

Die Institutionen des Clusters Forst und Holz in Niedersachsen sind damit aufgefordert, sich den Herausforderungen zu stellen, gemeinsame Ziele zu identifizieren und Strategien zu entwickeln, um sie zu erreichen. Dabei geht es im Prinzip um nicht mehr oder weniger, als um eine Konkretisierung der ökonomischen, ökologischen und sozialen Nachhaltigkeit der Produktion, Nutzung und Verwertung des Rohstoffes Holz in Niedersachsen. 


\section{Literatur}

ANONYMUS (1997): Ertragslage im Holzgewerbe hat sich deutlich verbessert. Holz-Zentralblatt, 123, 2370

ANONYMUS (2007): Mehr Beschäftigte im Holzgewerbe. Holz-Zentralblatt, 133, 305

BAYERISCHE LANDESANSTALT FÜR WALD UND FORSTWIRTSCHAFT (LWF) (Hrsg.) (2005): Holzaufkommensprognose für Bayern. LWF Wissen, Heft 50, $72 \mathrm{~S}$.

BAYERISCHES STAATSMINISTERIUM FÜR LANDWIRTSCHAFT UND FORSTEN und ZENTRUM WALD-FORST-HOLZ WeIHENSTEPHAN (Hrsg.) (2006): Cluster Forst und Holz - Bedeutung und Chancen für Bayern. München, Weihenstephan, $42 \mathrm{~S}$.

BECKEMAN, C.-G. U. LUUKKO K. (2005): Innovative and sustainable use of forest resources. Brüssel: European Confederation of Woodworking Industries, Confederation of European Forest Owners and Confederation of European Paper Industries, 19 S.

Bund Deutscher ZimMERMEISTER (Hrsg.) (2006): Bericht zur Lage der Zimmerer-Branche 2006. 4 S., http://www.bdz-holzbau.de/documents/Lagebericht.pdf

BUNDESMINISTERIUM FÜR ERNÄHRUNG, LANDWIRTSCHAFT UND VERBRAUCHERSCHUTZ (Hrsg.) (2005): Das potenzielle Rohholzaufkommen 2003 bis 2042. Das Wichtigste in Kürze. Bonn

BUNDESMINISTERIUM FÜR VERBRAUCHERSCHUTZ, ERNÄHRUNG UND LANDWIRTSCHAFT (Hrsg.) (2001): Aufnahmeanweisung für die Bundeswaldinventur II (2001 - 2002). 2. korrigierte, überarbeitete Auflage, Bonn, $108 \mathrm{~S}$.

BUNDESMINISTERIUM FÜR VERBRAUCHERSCHUTZ, ERNÄHRUNG UND LANDWIRTSCHAFT (Hrsg.) (2004): Die zweite Bundeswaldinventur - BWI 2. Das Wichtigste in Kürze. Bonn, $87 \mathrm{~S}$.

BUNDESRAT (Hrsg.) (2001): Entschließung des europäischen Parlaments zu der Mitteilung der Kommission über den Stand der Wettbewerbsfähigkeit der Holz verarbeitenden Industrie und Verwandter Industriezweige in der EU. Bundesdrucksache 113/01, $10 \mathrm{~S}$.

DiETER, M. (2007): mündl. Mitteilung

DiETER, M. u. ENGLERT, H. (2005): Gegenüberstellung und forstpolitische Diskussion unterschiedlicher Holzeinschlagsschätzungen für die Bundes- 
republik Deutschland. Institut für Ökonomie der Bundesforschungsanstalt für Forst- und Holzwirtschaft, Hamburg, Arbeitsbericht 2005/2, 10 S., http://www.bfafh.de/bibl/pdf/iii_05 02.pdf

Dieter, M.; Rosin, A. u. Thoroe, C. (2004): Die Forstwirtschaftliche Gesamtrechnung der Bundesrepublik Deutschland im Rahmen der ESVG 1995 für die Jahre 1991 bis 2002. Institut für Ökonomie der Bundesforschungsanstalt für Forst- und Holzwirtschaft (BFH), Hamburg, Arbeitsbericht 2004/15, 72 S.

Dieter, M. u. Thoroe, C. (2003): Forst- und Holzwirtschaft in der Bundesrepublik Deutschland nach neuer europäischer Sektorenabgrenzung. Forstwissenschaftliches Centralblatt, 122, 138-151

DUDA, H. (2006): Vergleich forstlicher Managementstrategien. Umsetzung verschiedener Waldbaukonzepte in einem Waldwachstumssimulator. Dissertation, Georg-August-Universität Göttingen (http://webdoc.sub.gwdg. de/diss/2006/duda/duda.pdf); Books on Demand GmbH, Norderstedt, $182 \mathrm{~S}$.

ESVG (1995): Europäisches System volkswirtschaftlicher Gesamtrechnungen. http://circa.europa.eu/irc/dsis/nfaccount/info/data/ESA95/de/esa95de.htm

FSC (2007): FSC-zertifizierte Wälder in Deutschland. www.fsc-deutschland.de, 13.02.2007.

HeIDER, G. (2005): Hohes Mengenwachstum in der Sägeindustrie. Die deutsche Sägeindustrie am Jahresende 2005. Presseinfo vom 31.12.2005 des Verbandes der Deutschen Säge- und Holzindustrie e.V., Wiesbaden, 6 S., http://www. saegeindustrie.de/news.cfm?ID $=337$

HoElzEL, M. (2007): Eine gute Lösung für alle zeichnet sich nicht ab. HolzZentralblatt, 133, 363-364

KRALEMANN, M. (2007a): Nutzung von Holzbrennstoffen in kleinen und mittleren Feuerungsanlagen. Tagung „Rohholzmanagement in Deutschland“, Kompetenznetz für Nachhaltige Holznutzung e. V. (NHN), Tagungsband (CD-ROM)

KRALEMANN, M. (2007b): Schriftliche Mitteilung

MANTAU, U. (2007): Energetische und stoffliche Holzverbrauchsentwicklung in Deutschland. Tagung „Rohholzmanagement in Deutschland“, Kompetenznetz für Nachhaltige Holznutzung e. V. (NHN), Tagungsband (CD-ROM)

MANTAU, U. u. SÖRGEL, C. (2004): Standorte der Holzwirtschaft - Holzwerkstoffindustrie, Holzschliff- und Zellstoffindustrie, Sägeindustrie. Universität Hamburg, Zentrum Holzwirtschaft, Arbeitsbereich: Ökonomie der Holz und Forstwirtschaft, Hamburg, 2004. 
MANTAU, U. u. SÖRGEL, C. (2006): Energieholzverwendung in privaten Haushalten. Marktvolumen und verwendete Holzsortimente - Zwischenbericht vom 06.07.2006. Universität Hamburg, Zentrum Holzwirtschaft, Arbeitsbereich: Ökonomie der Holz und Forstwirtschaft, Hamburg, 18 S.

MosReK, T. et. al. (2005): Clusterstudie Forst und Holz Deutschland 2005. HolzZentralblatt, 131, 1113 u. 1116-1117

NAGEL, J. (2005): TreeGrOSS - eine Java basierte Softwarekomponente zur Waldwachstumsmodellierung für Forschung, Lehre und Praxis. Tagungsband der 15. Jahrestagung der Sektion Forstliche Biometrie und Informatik im DVFFA vom 9.-10. Oktober 2003 in Freiburg, 33-37

NAGEL, J.; DUDA, H. u. HANSEN, J. (2006): Forest Simulator BWINPro7. Forst u. Holz, 61, 427-429

NiEDERSÄCHSISCHES MINISTERIUM FÜR DEN LÄNDLICHEN RAUM, ERNÄHRUNG, LANDWIRTSCHAFT UND VERBRAUCHERSCHUTZ (Hrsg.) (2004): Der Wald in Niedersachsen. Ergebnisse der Bundeswaldinventur II. Aus dem Walde - Schriftenreihe Waldentwicklung in Niedersachsen, Heft 55, 43 S.

REgIONALE PEFC-ARBEITSGRUPPE NiEDERSACHSEN (Hrsg.) (2005): Regionaler Waldbericht Niedersachsen 2005. Egestorf, 158 S.

Polley, H. u. Kroiher, F. (2006): Struktur und regionale Verteilung des Holzvorrates und des potenziellen Rohholzaufkommens in Deutschland im Rahmen der Clusterstudie Forst- und Holzwirtschaft. Institut für Waldökologie und Waldinventuren der Bundesforschungsanstalt für Forst- und Holzwirtschaft, Eberswalde, Arbeitsbericht 2006/3, 128 S.

SCHÄFER, V. (2004): Heizkraftwerke. In: CENTRALES AgRAR-ROHSTOFF-MARKETING- UND ENTWICKLUNGS-NETZWERK E. V. (Hrsg.): Nachwachsende Rohstoffe - Wirtschaftsfaktor Biomasse. C.A.R.M.E.N-Jahrbuch 2004/2005, Straubing, 63

Seegmüller, S. (2005): Die Forst-, Holz- und Papierwirtschaft in RheinlandPfalz. Struktur- und Genehmigungsdirektion Süd, Forschungsanstalt für Waldökologie und Forstwirtschaft und Ministerium für Umwelt und Forsten Rheinland-Pfalz (Hrsg.), Mitteilungen aus der Forschungsanstalt für Waldökologie und Forstwirtschaft Rheinland-Pfalz, Nr. 57/05, 67 S.

SÖRGEL, C. u. MANTAU, U. (2006a): Strukturentwicklung der Sägeindustrie in vier Jahren. Holz-Zentralblatt, 132, 651-653

SÖRGEL; C. u. MANTAU, U. (2006b): Standort der Holzwirtschaft - Holzwerkstoffindustrie-Abschlussbericht. Universität Hamburg, Zentrum Holzwirtschaft, Arbeitsbereich Ökonomie der Holz- und Forstwirtschaft, Hamburg, 30 S. 
STATISTISCHES BundeSAMT (Hrsg.) (2003): Klassifikation der Wirtschaftszweige mit Erläuterungen, Ausgabe 2003. Wiesbaden, 860 S., www.statistikportal.de/Statistik-Portal/klassiWZ03.pdf

STATISTISCHES BUNDESAMT (2007): Volkswirtschaftliche Gesamtrechnungen Wichtige Zusammenhänge im Überblick - 2006. Wiesbaden, 29 S., www.destatis.de/jetspeed/portal/cms/Sites/destatis/Internet/DE/Content/Publikati onen/Fachveroeffentlichungen/VolkswirtschaftlicheGesamtrechnungen/Zusammenh aenge,property $=$ file.pdf

STATISTISCHES LANDESAMT NiEDERSACHSEN (2006): http://www1.nls. niedersachsen.de/statistik/

THÜRINGER MINISTERIUM FÜR LANDWIRTSCHAFT, NATURSCHUTZ UND UMWELT (Hrsg.) (2007): Cluster Forst und Holz - Chancen für Thüringen. Erfurt, $23 \mathrm{~S}$.

WEBER, H. (2001): Strategische Geschäftsfeldplanung in Unternehmen der Sägeindustrie - unter Berücksichtigung der Unternehmensumwelt, insbesondere von Konzentration und Diversifikation. Dissertation Universität Freiburg, $350 \mathrm{~S}$.

Zentrale MarkT- und PReisberichtsstelle GmbH (ZMP) (2005): ZMPMarktbilanz Forst und Holz 2005. Bonn

ZENTRALE MARKT- UND PREISBERICHTSSTELLE GmbH (ZMP) (2006): ZMPMarktbilanz Forst und Holz 2006. Bonn, 175 S. 


\section{Glossar}

Arbeitnehmer: Als Arbeitnehmer zählt, wer zeitlich überwiegend als Arbeiter, Angestellter, Beamter, Richter, Berufssoldat, Auszubildender, Praktikant oder Volontär in einem Arbeits- bzw. Dienstverhältnis steht. Eingeschlossen sind auch Heimarbeiter und geringfügig Beschäftigte.

Arbeitnehmerentgelte: Das Arbeitnehmerentgelt umfasst sämtliche Geld- und Sachleistungen, die von einem Arbeitgeber an einen Arbeitnehmer erbracht werden, und zwar als Entgelt für die von diesem Darstellungszeitraum geleistete Arbeit.

Bruttoproduktionswert: Der Bruttoproduktionswert ist eine Ertragsgröße der unternehmerischen Wertschöpfung. Er misst den tatsächlichen Produktionsumfang auf der Grundlage der Umsatzerlöse, der Vorratsränderungen und des Wiederverkaufs von Waren und Dienstleistungen.

Bruttowertschöpfung: Die Bruttowertschöpfung berechnet sich aus der Differenz des Bruttoproduktionswertes zu Herstellungspreisen und den Vorleistungen zu Käuferpreisen. Die Wertschöpfung enthält somit nicht die Gütersteuern, jedoch die Gütersubventionen. Die Bruttowertschöpfung wird vor Abzug der Abschreibungen ausgewiesen.

Bundeswaldinventur 2, BWI 2: Bundesweite Erhebung des BMVEL zur Erfassung großräumiger Waldverhältnisse und forstlicher Produktionsmöglichkeiten in den Jahren 2001 bis 2002 (BMVEL 2004).

TreeGrOSS: TreeGrOSS: Tree Growth Open Source Software, früher BWINPro, frei verfügbares JAVA-Softwarepaket zur einzelbaumbasierten Simulation von Bestandsentwicklungen (Nagel et al. 2006).

Vorleistungen: Die Vorleistungen messen den Wert der im Produktionsprozess verbrauchten, verarbeiteten oder umgewandelten Waren und Dienstleistungen. Nicht dazu gehört die Nutzung des Anlagevermögens, die anhand der Abschreibung gemessen werden.

WEHAM oder auch HAM: Holzaufkommensmodellierung. Bundesweite Holzaufkommensprognose auf Basis der Bundeswaldinventur (Basisszenario) im Auftrag des BMELV. 


\title{
Autoren
}

\section{Burkhard Rüther}

Øvre Fritzøegate 41

N-3264 Larvik

Norwegen

burkhard.ruether@mac.com

\section{Agatha Ludwig}

Georg-August-Universität Göttingen

Burkhardt-Institut

Abteilung für Forstökonomie u.

Forsteinrichtung

Büsgenweg 5

D-37077 Göttingen

\section{Prof. Dr. Jürgen Nagel}

Nordwestdeutsche Forstliche

Versuchsanstalt

Grätzelstr. 2

D-37079 Göttingen

Juergen.Nagel@,nw-fva.de

\section{Jan Hansen}

Nordwestdeutsche Forstliche

Versuchsanstalt

Grätzelstr. 2

D-37079 Göttingen

Jan.Hansen@,nw-fva.de

\section{Prof. Dr. Hermann Spellmann}

Nordwestdeutsche Forstliche

Versuchsanstalt

Grätzelstr. 2

D-37079 Göttingen

Hermann.Spellmann@,nw-fva.de

\author{
Prof. Dr. Bernhard Möhring \\ Georg-August-Universität Göttingen \\ Burkhardt-Institut \\ Abteilung für Forstökonomie u. \\ Forsteinrichtung \\ Büsgenweg 5 \\ D-37077 Göttingen \\ bmoehri@uni-forst.gwdg.de
}

\author{
Dr. Matthias Dieter \\ Bundesforschungsanstalt für Forst- und \\ Holzwirtschaft (BFH) \\ Institut für Ökonomie \\ Fachgebiet Holzmarktforschung \\ Leuschnerstr. 91 \\ D-21031 Hamburg \\ m.dieter@holz.uni-hamburg.de
}


Die Globalisierung der Rohstoff- und Warenmärkte stellt die deutsche Forst- und Holzwirtschaft vor neue Herausforderungen. Sie hat zu einer steigenden Holznachfrage und zum Aufbau neuer Produktionskapazitäten der Holzindustrie geführt. Ziel der Clusterstudie Forst und Holz Niedersachsen ist es, die Wettbewerbsfähigkeit der niedersächsischen Forst- und Holzwirtschaft vor diesem Hintergrund zu stärken. Hierzu wurden die inneren Strukturen (Betriebe, Umsätze, Beschäftigungszahlen) und wirtschaftlichen Potenziale analysiert, die möglichen Holzaufkommen bei Unterstellung verschiedener Bewirtschaftungsstrategien für einen Zeitraum von 30 Jahren prognostiziert und die wesentlichen Einflussfaktoren auf das zu erwartende Nutzungsverhalten der Forstbetriebe aufgezeigt. Die Clusterstudie Forst und Holz Niedersachsen richtet sich an alle dem Cluster angehörenden Betriebe der Forst- und Holzwirtschaft sowie an die nachgelagerten Bereiche, die Verwaltungen, Forschungseinrichtungen und die Politik. Auf der Basis dieser Studie können gemeinsame Strategien der Forst- und Holzwirtschaft entwickelt werden. 